\title{
Kleinian groups of small Hausdorff dimension are classical Schottky groups. I
}

\author{
YONG HOU
}

\begin{abstract}
It has been conjectured that the Hausdorff dimensions of nonclassical Schottky groups are strictly bounded from below. In this first part of our work on this conjecture, we prove that there exists a universal positive number $\lambda>0$ such that any 2-generated nonelementary Kleinian group with limit set of Hausdorff dimension $<\lambda$ is a classical Schottky group.
\end{abstract}

57M50, 57M05; 53C21, 53C20, 37A15

\section{Introduction and Main Theorem}

Let $\mathbb{H}^{3}$ be hyperbolic 3 -space. A subgroup $\Gamma$ of $\operatorname{PSL}(2, \mathbb{C})=\operatorname{Iso}\left(\mathbb{H}^{3}\right)$ is called a Kleinian group if it is discrete. Let $x \in \mathbb{H}^{3}$. The orbit of $x$ under the action of $\Gamma$ is denoted by $\Gamma x$. The limit set $\Lambda_{\Gamma}$ of $\Gamma$ is defined as $\Lambda_{\Gamma}=\overline{\Gamma x} \cap \partial \mathbb{H}^{3}$. By definition, $\Lambda_{G}$ is the smallest closed $\Gamma$-invariant subset of $\partial \mathbb{H}^{3}$. The group $\Gamma$ is said to be elementary if $\Lambda_{G}$ contains at most two points, otherwise $\Gamma$ is said to be nonelementary. Note that elementary Kleinian groups are completely classified. Henceforth when we say "Kleinian group $\Gamma$ " we will assume that $\Gamma$ is nonelementary. The group $\Gamma$ is of the second kind if $\Lambda_{\Gamma} \neq \partial \mathbb{H}^{3}$, otherwise it is said to be of the first kind. The set $\Omega_{\Gamma}=\partial \mathbb{H}^{3}-\Lambda_{\Gamma}$ is the region of discontinuity, and $\Gamma$ acts properly discontinuously on $\Omega_{\Gamma}$.

Let $\left\{\Delta_{1}, \Delta_{1}^{\prime}, \ldots, \Delta_{k}, \Delta_{k}^{\prime}\right\}$ be a collection of disjoint closed Jordan curves in the Riemann sphere $\overline{\mathbb{C}}$ and let $D_{i}, D_{i}^{\prime}$ be the topological disks bounded by $\Delta_{i}, \Delta_{i}^{\prime}$ respectively. Suppose we have $\left\{\gamma_{i}\right\}_{1}^{k} \subset \operatorname{PSL}(2, \mathbb{C})$ such that $\gamma_{i}\left(\Delta_{i}\right)=\Delta_{i}^{\prime}$ and $\gamma_{i}\left(D_{i}\right) \cap D_{i}^{\prime}=\varnothing$. Then the group $\Gamma$ generated by $\left\{\gamma_{1}, \ldots, \gamma_{k}\right\}$ is a free Kleinian group of rank $k$, and $\Gamma$ is called a marked Schottky group with marking $\left\{\gamma_{1}, \ldots, \gamma_{k}\right\}$. A finitely generated Kleinian group $\Gamma$ is called a Schottky group if it is a marked Schottky group for some marking. If there exists a generating set $\left\{\gamma_{1}, \ldots, \gamma_{k}\right\}$ such that all $\Delta_{i}, \Delta_{i}^{\prime}$ can be taken as circles then it is called a marked classical Schottky group with classical marking $\left\{\gamma_{1}, \ldots, \gamma_{k}\right\}$, and $\left\{\gamma_{1}, \ldots, \gamma_{k}\right\}$ are called classical generators. A Schottky group $\Gamma$ is called classical Schottky group if there exists a classical marking for $\Gamma$. 
For a Schottky group $\Gamma$, the manifold $\mathbb{H}^{3} / \Gamma$ is homeomorphic to the interior of a handlebody of genus $k$. We denote by $\mathfrak{J}_{k}$ the set of all rank $k$ Schottky groups, and let $\mathfrak{J}_{k, o}$ be the set of all rank $k$ classical Schottky groups. One simple way to topologize $\mathfrak{J}_{k}$ is to identify it with the space of moduli of a Riemann surface of genus $k$.

It is known that not all Schottky groups are classical Schottky groups. In fact, the space of classical Schottky groups is not even dense in the space of Schottky groups by Marden [5]; also see Doyle [3].

In [3], Peter Doyle proved that there exists a universal upper bound on the Hausdorff dimension of the limit sets of finitely generated classical Schottky groups. It was originally Phillips and Sarnak in [7] who proved that there exists a universal upper bound on the Hausdorff dimension of the limit sets of classical Schottky groups of dimension greater than 3 .

Let $\mathfrak{D}_{\Gamma}$ denote the Hausdorff dimension of $\Lambda_{\Gamma}$. The main result is the following.

Theorem 1.1 There exists a universal $\lambda>0$ such that any 2-generated nonelementary Kleinian group $\Gamma$ with $\mathfrak{D}_{\Gamma}<\lambda$ is a classical Schottky group.

Note that our result can be viewed as the converse of the result by Doyle [3] and Phillips and Sarnak [7]. The proofs of their theorems rely on the crucial fact that $\lambda_{0}\left(\mathbb{H}^{n+1} / \Gamma\right)=\mathfrak{D}_{\Gamma}\left(n-\mathfrak{D}_{\Gamma}\right)$, for $\mathfrak{D}_{\Gamma} \geq 1$, where $\lambda_{0}\left(\mathbb{H}^{n+1} / \Gamma\right)$ is the bottom spectrum of the Laplacian of the hyperbolic manifold $\mathbb{H}^{n+1} / \Gamma$. But this identity obviously is useless in our situation.

We prove Theorem 1.1 by using a result from our paper [4] and selections of generators. The proof is divided into three main steps.

To lead up to the proof, we first do some preliminary estimates on the locations of the fixed points of a given set of generators of a Schottky group. These estimates give us a sufficient control on how the fixed points of a set of generators change in terms of the Hausdorff dimension of the limit set of the group. The main ingredient of the proofs of these estimates relies on [4, Theorem 1.1], rewritten in the trace form.

Next we obtain a set of sufficient conditions for any given sequence of Schottky groups to contain a subsequence of classical Schottky groups in the unit ball in hyperbolic space. These conditions are stated in the upper-half space hyperbolic model. The idea is that if the radius of isometric circles of a sequence of generators decreases sufficiently faster than the reduction of the gaps between any of the fixed points of the sequence of generators, then this sequence of generators will eventually become classical generators. We do this first by transforming the generators with the condition that the generator 
with the shortest translation length is transformed into vertical position passing through the origin with fixed points at north and south poles. And then these generators are projected into upper-half space.

In the first step of the proof, we consider Schottky subspaces of the Schottky space that consist of Schottky groups $\Gamma$ for which there exists a generating set $S_{\Gamma}$ for $\Gamma$ with the set of fixed points of $S_{\Gamma}$ on the boundary sphere on the unit 3-ball hyperbolic space that are mutually bounded away from each other by a positive constant.

In this step we prove that Theorem 1.1 holds for these Schottky subspaces. This is proved via contradiction. Suppose $\Gamma_{n}$ is a sequence of nonclassical Schottky groups in the subspace with the Hausdorff dimension of $\Lambda_{\Gamma_{n}}$ decreasing to 0 .

The idea is that we first transform these generators of the generating sets $S_{\Gamma_{n}}$ into the standard form with the generator of $S_{\Gamma_{n}}$ of shortest translation length put in vertical position. If no generator of $S_{\Gamma_{n}}$ is of bounded translation length when $\mathfrak{D}_{\Gamma_{n}} \rightarrow 0$ then it's easy to see this will lead to a contradiction. On the other hand, it's a simple corollary of [4] that there can exists at most one generator of bounded translation length per $S_{\Gamma_{n}}$ when $\mathfrak{D}_{\Gamma_{n}} \rightarrow 0$. If such a generator does exists, then we first make a careful change of generators which will be constructed based on estimates that its fixed-point set will be "minimally excluded" from isometric spheres of the generator of bounded translation length. Working with these generating sets we will show that with appropriate additional transformations and changes, the generators with translation length that is not bounded will always grow sufficiently fast to lead to disjoint isometric spheres which are also disjoint from the rest of the isometric spheres of the other generators.

One of the crucial tools in estimating this growth is the strong form of the inequality of [4]. We will show that when one of the generators is of bounded translation length then it will force the needed growth for the rest of the generators. With appropriate choice of generators this will lead to a classical Schottky group.

In the second step, we prove that on the nonclassical Schottky space there exist universal lower bounds for $\mathfrak{D}_{\Gamma}+Z_{\Gamma}$; see Section 2 for notation and definitions. Essentially this means that we cannot simultaneously have arbitrarily small Hausdorff dimension and minimal gaps of the fixed-point set on the space of nonclassical Schottky groups. This is proved using generator selection and the results of step one.

In the last step, we prove that when $\mathfrak{D}_{\Gamma}$ is taken sufficiently small then $\Gamma$ can be taken as Schottky group. This is based on basic topological arguments and some well known results on Kleinian groups. We prove (by an easy standard topological argument) that any finitely generated Kleinian group with limit set of sufficiently small 
Hausdorff dimension is a Schottky group, but Dick Canary pointed out that it's simpler in the 2-generated case based on the result of Peter Shalen which assures that any 2-generated Kleinian group is either free or cofinite volume.

The paper is organized as follows: In Section 2, we define and list global notation which will be used throughout the paper. In Section 3, a strong form of Theorem 1.1 of [4] will be stated for two generators, which we shall use for selecting generators; see Corollary 3.2. In Section 4, for a given sequence of Schottky groups $\Gamma_{n}$ with bounds on $Z_{\Gamma_{n}}$ (see Section 2), we prove inequalities that will enable us to control fixed points of a given sequence of generators of $\Gamma_{n}$ in relation to the Hausdorff dimensions $\mathfrak{D}_{\Gamma_{n}}$ of $\Gamma_{n}$. These will be used in the selection process. In Section 5, sufficient conditions for a given pair of generators to be classical generators are established which we will use in our generator selection process. In Section 6, we will use tools developed in previous sections to form a generator selection process and prove that Schottky groups with small $\mathfrak{D}_{\Gamma_{n}}$ and bounds on $Z_{\Gamma_{n}}$ are classical Schottky groups. In Section 7, we will prove the theorem that will remove the bound condition on $Z_{\Gamma_{n}}$. Section 8 completes the proof of our main theorem by reducing finitely generated Kleinian groups with small Hausdorff dimensions to Schottky groups via a standard known topological argument. This paper is dedicated to Ying Zhou.

Acknowledgements I wish to express my deepest appreciation to the referee for spending enormous amount of time reading and correcting the paper which made the paper much better. I would like to express my sincere appreciation and gratitude to Benson Farb for providing me guidance and support in the write-up of the paper. I would like to express my gratitude to Jim Anderson, Peter Shalen, Marc Culler and Dick Canary for their interest in the paper. I am indebted to Ying Zhou for all the support and unwavering help provided to me during this work.

\section{Notation}

Let $\Gamma \subset \operatorname{PSL}(2, \mathbb{C})$ denote a Schottky group generated by $\langle\alpha, \beta\rangle$ with $\alpha$ having fixed points $0, \infty$. Assume that $\gamma \in \Gamma$ is a loxodromic element having fixed points $\neq \infty$. Write $\gamma$ in matrix form

$$
\gamma=\left(\begin{array}{ll}
a & b \\
c & d
\end{array}\right)
$$

with $\operatorname{det}(\gamma)=1$. We will set the following notation and definitions throughout the rest of the paper.

Notation 1 - Denote the critical exponent of $\Gamma$ by $D_{\Gamma}$ and the Hausdorff dimension of $\Lambda_{\Gamma}$ by $\mathfrak{D}_{\Gamma}$. 
- $\mathfrak{R}_{\gamma}$ denotes the radius of isometric circles of $\gamma$.

- $\eta_{\gamma}=-d / c, \zeta_{\gamma}=a / c$.

- We will define two different ways to denote the two fixed points of $\gamma:\left\{z_{\gamma, l}, z_{\gamma, u}\right\}$ denotes the two fixed points of $\gamma$ in $\mathbb{C}$ with $\left|z_{\gamma, l}\right| \leq\left|z_{\gamma, u}\right|$ and $\left\{z_{\gamma,-}, z_{\gamma,+}\right\}$ denotes the two fixed points with $z_{\gamma, \pm}$ given by quadratic formula with subscripts \pm corresponding to $\pm \sqrt{\operatorname{tr}^{2}(\gamma)-4}$. Note that we always take the principle branch for the square roots of complex numbers.

- $\mathcal{L}_{\gamma}$ is the axis of $\gamma$.

- $T_{\gamma}$ is the translation length of $\gamma$.

- Set $Z_{\beta}:=\min \left\{\left|z_{\beta,-}-z_{\beta,+}\right|,\left|1 / z_{\beta,-}-1 / z_{\beta,+}\right|\right\}$.

- $Z_{\langle\alpha, \beta\rangle}:=\min \left\{Z_{\beta},\left|z_{\beta,+}\right|,\left|z_{\beta,-}\right|,\left|z_{\beta,+}\right|^{-1},\left|z_{\beta,-}\right|^{-1}\right\}$.

- For $\epsilon>0$, we say that $Z_{\Gamma}>\epsilon$, if there exists a generating set $\langle\alpha, \beta\rangle$ of $\Gamma$ such that $Z_{\langle\alpha, \beta\rangle}>\epsilon$.

- Given any two sequences of real numbers $\left\{p_{n}, q_{n}\right\}$, the notation $p_{n} \asymp q_{n}$ means there exists $\sigma>0$ such that $\sigma^{-1}<\liminf p_{n} / q_{n} \leq \limsup p_{n} / q_{n}<\sigma$.

Notation 2 Let $\left\{\gamma_{n}\right\} \subset \operatorname{PSL}(2, \mathbb{C})$ be a sequence of loxodromic transformations. Let $\left\{p_{n}\right\}$ be a sequence of complex numbers, and $\left\{q_{n}\right\}$ a sequence of positive real numbers. We write

$$
\left|z_{\gamma_{n}, \pm}-p_{n}\right|<q_{n}
$$

if there exists $N$ such that for every $n>N$ we have at least one of the following holds:

(i) $\left|z_{\gamma_{n},+}-p_{n}\right|<q_{n}$.

(ii) $\left|z_{\gamma_{n},-}-p_{n}\right|<q_{n}$.

\section{Free group actions}

Given a finitely generated nonelementary Kleinian group $\Gamma$, the critical exponent of $\Gamma$ is the unique positive number $D_{\Gamma}$ such that the Poincare series of $\Gamma$ given by $\sum_{\gamma \in \Gamma} e^{-s \operatorname{dist}(x, \gamma x)}$ is divergent if $s<D_{\Gamma}$ and convergent if $s>D_{\Gamma}$. If the Poincaré series diverges at $s=D_{\Gamma}$ then $\Gamma$ is said to be divergent. Bishop-Jones showed that $D_{\Gamma} \leq \mathfrak{D}_{\Gamma}$ for all analytically finite nonelementary Kleinian groups $\Gamma$. In fact, if $\Gamma$ is topologically tame $\left(\mathbb{H}^{3} / \Gamma\right.$ homeomorphic to the interior of a compact manifold-withboundary) then $D_{\Gamma}=\mathfrak{D}_{\Gamma}$. Hence it follows Agol's [1] proof of tameness conjecture that $D_{\Gamma}=\mathfrak{D}_{\Gamma}$ for all finitely generated nonelementary Kleinian groups. The critical 
exponent is a geometrically rigid object in the sense that a decrease in $D_{\Gamma}$ corresponds to a decrease in geometric complexity.

Next we state the following theorem from [4], which provides the relation between the group action and the critical exponent.

Theorem 3.1 (Hou [4]) Let $\Gamma$ be a free nonelementary Kleinian group of rank $k$ with free generating set $\mathcal{S}$, and $x \in \mathbb{H}^{3}$ then

$$
\sum_{\gamma \in \mathcal{S}} \frac{1}{1+\exp \left(D_{\Gamma} \operatorname{dist}(x, \gamma x)\right)} \leq \frac{1}{2} .
$$

In particular we have at least $k-1$ distinct elements $\left\{\gamma_{i_{j}}\right\}_{1 \leq j \leq k-1}$ of $\mathcal{S}$ that satisfies $\operatorname{dist}\left(x, \gamma_{i_{j}} x\right) \geq \log (3) / D_{\Gamma}$, and at least one element $\gamma_{i_{j}}$ with $\operatorname{dist}\left(x, \gamma_{i_{j}} x\right) \geq$ $\log (2 k-1) / D_{\Gamma}$.

The following is a useful corollary of Theorem 3.1, stated here for the case of $\Gamma$ is a free group of rank 2 .

Corollary 3.2 Let $\mathcal{S}=\left\{\gamma_{1}, \gamma_{2}\right\}$ be a generating set for a free nonelementary Kleinian group $\Gamma$. Let $x \in \mathbb{H}^{3}$. Then

$$
\operatorname{dist}\left(x, \gamma_{2} x\right) \geq \frac{1}{D_{\Gamma}} \log \left(\frac{e^{D_{\Gamma} \operatorname{dist}\left(x, \gamma_{1} x\right)}+3}{e^{D_{\Gamma} \operatorname{dist}\left(x, \gamma_{1} x\right)}-1}\right)
$$

Corollary 3.3 Let $\mathcal{S}=\left\{\gamma_{1}, \gamma_{2}\right\}$ be a generating set for a free nonelementary Kleinian group $\Gamma$. Let $x \in \mathbb{H}^{3}$. Let $m$ be any integer. Then at least one of the elements $\gamma^{\prime}$ of $\mathcal{S}^{\prime}=\left\{\gamma_{1}^{m} \gamma_{2}, \gamma_{1}^{m+1} \gamma_{2}\right\}$ satisfies $\operatorname{dist}\left(x, \gamma^{\prime} x\right) \geq \log 3 / D_{\Gamma}$.

\section{Trace, fixed points and Hausdorff dimension}

In this section we study the relationships of fixed points of generating sets of a given sequence of Schottky groups $\Gamma_{n}$ and the Hausdorff dimensions of $\Lambda_{\Gamma_{n}}$.

What we like to do is to find a relationship between the distribution of the fixed points of one of the generators in terms of the translation length growth of the other generator and the Hausdorff dimension of its limit set. By having this type of relationship we will be able to construct a new set of generators from the given generating set with prescribed distribution of its fixed points. The new set of generators will be a crucial ingredient in the proof of our theorem. 
Let $\left\{\Gamma_{n}\right\}$ be a sequence of rank 2 Schottky groups with $D_{n} \rightarrow 0$ generated by $\alpha_{n}, \beta_{n} \in$ $\operatorname{PSL}(2, \mathbb{C})$ in the upper space model $\mathbb{H}^{3}$ with

Set

$$
\begin{aligned}
\alpha_{n} & =\left(\begin{array}{cc}
\lambda_{n} & 0 \\
0 & \lambda_{n}^{-1}
\end{array}\right), \quad\left|\lambda_{n}\right|>1 . \\
\beta_{n} & =\left(\begin{array}{ll}
a_{n} & b_{n} \\
c_{n} & d_{n}
\end{array}\right) .
\end{aligned}
$$

We assume throughout this section that there exists $M>0$ such that $T_{\alpha_{n}}<M$ for all $n$. Set $D_{n}=D_{\Gamma_{n}}$. Let $\operatorname{tr}=$ trace.

First we will state Corollary 3.2 in the trace form.

Proposition 4.1 Suppose there exists $\Delta>0$ such that $Z_{\beta_{n}}>\Delta$. There exists $\rho>0$ depending on $\Delta$ such that

$$
\left|\operatorname{tr}\left(\beta_{n}\right)\right|>\rho\left(\frac{\left|\lambda_{n}\right|^{2 D_{n}}+3}{\left|\lambda_{n}\right|^{2 D_{n}}-1}\right)^{1 /\left(2 D_{n}\right)}
$$

for large $n$.

Proof Let $T_{n}$ be the translation length of $\beta_{n}$ and $R_{n}=\operatorname{dist}\left(\mathcal{L}_{\alpha_{n}}, \mathcal{L}_{\beta_{n}}\right)$. Let $x_{n}$ be a point on axis of $\alpha_{n}$ which is the nearest point of $\mathcal{L}_{\alpha_{n}}$ to $\mathcal{L}_{\beta_{n}}$. By triangle inequality, $T_{n} \geq \operatorname{dist}\left(x_{n}, \beta_{n} x_{n}\right)-2 R_{n}$. And for sufficiently large $T_{n}$, we have for some positive constant $c>0,\left|\operatorname{tr}^{2}\left(\beta_{n}\right)\right|>c e^{T_{n}}$. Now for large $n$, from Corollary 3.2,

$$
\left|\operatorname{tr}^{2}\left(\beta_{n}\right)\right|>c\left(\frac{\left|\lambda_{n}\right|^{2 D_{n}}+3}{\left|\lambda_{n}\right|^{2 D_{n}}-1}\right)^{1 / D_{n}}\left(e^{-2 \operatorname{dist}\left(\mathcal{L}_{\alpha_{n}}, \mathcal{L}_{\beta_{n}}\right)}\right) .
$$

Now $Z_{\beta_{n}}>\Delta$ implies that $\operatorname{dist}\left(\mathcal{L}_{\alpha_{n}}, \mathcal{L}_{\beta_{n}}\right)<M$ for some $M>0$. Hence the result follows.

Remark 4.1.A Note that without assuming bounds on $Z_{\beta_{n}}$ we can state above Proposition 4.1 as

$$
\left|\operatorname{tr}^{2}\left(\beta_{n}\right)\right|>c\left(\frac{\left|\lambda_{n}\right|^{2 D_{n}}+3}{\left|\lambda_{n}\right|^{2 D_{n}}-1}\right)^{1 / D_{n}}\left(e^{-2 \operatorname{dist}\left(\mathcal{L}_{\alpha_{n}}, \mathcal{L}_{\beta_{n}}\right)}\right) .
$$

If $\operatorname{dist}\left(\mathcal{L}_{\alpha_{n}}, \mathcal{L}_{\alpha_{n} \beta_{n}}\right)<\epsilon$ then there exists $\delta>0$ such that

$$
\left|\operatorname{tr}\left(\beta_{n}\right)\right|>\rho\left(\frac{\left|\lambda_{n}\right|^{2 D_{n}}+3}{\left|\lambda_{n}\right|^{2 D_{n}}-1}\right)^{1 /\left(2 D_{n}\right)}
$$

for large $n$. 
The next lemma and its corollaries are estimates of convergence rates of fixed points of the generators of $\Gamma_{n}$ in terms of the Hausdorff dimension of $\Lambda_{n}$.

Lemma 4.2 Suppose there exists $\Delta>0, M>0$ such that $Z_{\left\langle\alpha_{n}, \beta_{n}\right\rangle}>\Delta$ and $T_{\alpha_{n}}<M$ for all $n$. Let $k_{n}, l_{n}$ be any integers such that $T_{\alpha_{n} k_{n}}, T_{\alpha_{n} l_{n}}<M$. Then there exists a constant $\rho>0$ such that

$$
\left|z_{\alpha_{n}^{k n} \beta_{n} \alpha_{n}^{l_{n}}, \pm}-\zeta_{\alpha_{n}^{k_{n}} \beta_{n} \alpha_{n}^{l_{n}}}\right|+\left|z_{\alpha_{n}^{k_{n}} \beta_{n} \alpha_{n}^{l_{n}}, \mp}-\eta_{\alpha_{n}^{k_{n}} \beta_{n} \alpha_{n}^{l_{n}}}\right|<\frac{\rho}{\left|\operatorname{tr}\left(\beta_{n}\right)\right|\left|\lambda_{n}^{l_{n}-k_{n}}\right|}
$$

for large $n$.

Remark 4.3 Lemma 4.2 is an estimate of how fast the fixed points converges, it's not important to our applications in this paper which fixed point converges to $\eta_{\gamma}$ and which converges to $\zeta_{\gamma}$ for a given $\gamma \in \operatorname{PSL}(2, \mathbb{C})$.

Given a complex number $z=r e^{i \theta}$ we write $\sqrt{z^{2}}=z$ if $-\pi<2 \theta \leq \pi$, and $\sqrt{z^{2}}=-z$ if $2 \theta>\pi$ or $2 \theta \leq-\pi$. Then a more precise statement of Lemma 4.2 which dichotomizes the above inequality for large $n$ would be:

$$
\begin{aligned}
& \sqrt{\operatorname{tr}^{2}\left(\alpha_{n}^{k_{n}} \beta_{n} \alpha_{n}^{l_{n}}\right)}=\operatorname{tr}\left(\alpha_{n}^{k_{n}} \beta_{n} \alpha_{n}^{l_{n}}\right) \\
& \left|z_{\alpha_{n}^{k_{n}} \beta_{n} \alpha_{n}^{l_{n}},+}-\zeta_{\alpha_{n}^{k n} \beta_{n} \alpha_{n}^{l_{n}}}\right|+\left|z_{\alpha_{n}^{k_{n}} \beta_{n} \alpha_{n}^{l_{n}},-}-\eta_{\alpha_{n}^{k_{n}} \beta_{n} \alpha_{n}^{l_{n}}}\right|<\frac{\rho}{\left|\operatorname{tr}\left(\beta_{n}\right)\right|\left|\lambda_{n}^{l_{n}-k_{n}}\right|}
\end{aligned}
$$

(ii) $-\sqrt{\operatorname{tr}^{2}\left(\alpha_{n}^{k_{n}} \beta_{n} \alpha_{n}^{l_{n}}\right)}=\operatorname{tr}\left(\alpha_{n}^{k_{n}} \beta_{n} \alpha_{n}^{l_{n}}\right)$

$$
\left|z_{\alpha_{n}^{k n} \beta_{n} \alpha_{n}^{l_{n}},-}-\zeta_{\alpha_{n}^{k_{n}} \beta_{n} \alpha_{n}^{l_{n}}}\right|+\left|z_{\alpha_{n}^{k_{n}} \beta_{n} \alpha_{n}^{l_{n}},+}-\eta_{\alpha_{n}^{k_{n}} \beta_{n} \alpha_{n}^{l_{n}}}\right|<\frac{\rho}{\left|\operatorname{tr}\left(\beta_{n}\right)\right|\left|\lambda_{n}^{l_{n}-k_{n}}\right|}
$$

Corollary 4.4 Suppose there exists $\Delta>0, M>0$ such that $Z_{\left\langle\alpha_{n}, \beta_{n}\right\rangle}>\Delta$ and $T_{\alpha_{n}}<M$ for all $n$. Let $k_{n}, l_{n}$ be any integers such that $T_{\alpha_{n} k_{n}}, T_{\alpha_{n} l_{n}}<M$. Then for any $\delta>0$ there exists $\epsilon>0$ such that if $D_{n}<\epsilon$ then,

$$
\left|z_{\alpha_{n}^{k_{n}} \beta_{n} \alpha_{n}^{l_{n}}, \pm}-\zeta_{\alpha_{n}^{k_{n}} \beta_{n} \alpha_{n}^{l_{n}}}\right|+\left|z_{\alpha_{n}^{k_{n}} \beta_{n} \alpha_{n}^{l_{n}}, \mp}-\eta_{\alpha_{n}^{k_{n}} \beta_{n} \alpha_{n}^{l_{n}}}\right|<\delta\left(\left|\lambda_{n}\right|^{2}-1\right) .
$$

The same dichotomy decomposition of the inequality holds as given in Remark 4.3.

Proof Let us assume that $\sqrt{\operatorname{tr}^{2}\left(\alpha_{n}^{k_{n}} \beta_{n} \alpha_{n}^{l_{n}}\right)}=\operatorname{tr}\left(\alpha_{n}^{k_{n}} \beta_{n} \alpha_{n}^{l_{n}}\right)$. Also $Z_{\left\langle\alpha_{n}, \beta_{n}\right\rangle} \geq \Delta$ by Proposition 4.1 as $D_{n} \rightarrow 0$ we have $\left|\operatorname{tr}\left(\beta_{n}\right)\right| \rightarrow \infty$. In addition, by $Z_{\left\langle\alpha_{n}, \beta_{n}\right\rangle} \geq \Delta$, we have $\left|z_{+, \beta_{n}}-z_{-, \beta_{n}}\right| \geq \Delta$, and also $\left|z_{+, \beta_{n}}\right|^{-1},\left|z_{-, \beta_{n}}\right|^{-1} \geq \Delta$ gives $\left|z_{+, \beta_{n}}-z_{-, \beta_{n}}\right| \leq$ $\left|z_{+, \beta_{n}}\right|+\left|z_{-, \beta_{n}}\right|<2 / \Delta$. There exists $c_{1}, c_{2}>0$ with $c_{1}<\left|z_{+, \beta_{n}}-z_{-, \beta_{n}}\right|<c_{2}$. By $\left|z_{+, \beta_{n}}-z_{-, \beta_{n}}\right|=\left|\sqrt{\operatorname{tr}^{2}\left(\beta_{n}\right)-4} /\left(2 c_{n}\right)\right|$, such we have $c_{1}<\left|\sqrt{\operatorname{tr}^{2}\left(\beta_{n}\right)-4} /\left(2 c_{n}\right)\right|<c_{2}$. 
This implies $\left|\operatorname{tr}\left(\beta_{n}\right)\right| \asymp\left|c_{n}\right|$, and since $\operatorname{tr}\left(\beta_{n}\right)=a_{n}+d_{n}$, there exists $c_{3}, c_{4}>0$ such that $c_{3}<\left|\left(a_{n}+d_{n}\right) / c_{n}\right|<c_{4}$. Since $\left|\operatorname{tr}\left(\beta_{n}\right)\right| \rightarrow \infty$, implies $\Re_{\beta_{n}} \rightarrow 0$, and $Z_{\left\langle\alpha_{n}, \beta_{n}\right\rangle} \geq \Delta$ gives us that there exists $c_{5}, c_{6}>0$ such that $c_{5}<\left|a_{n} / c_{n}\right|,\left|d_{n} / c_{n}\right|<c_{6}$, for large $n$.

Therefore the fixed points $z_{ \pm, \beta_{n}}$ of $\beta_{n}$ must $\rightarrow\left\{a_{n} / c_{n},-d_{n} / c_{n}\right\}$. There exists $c, c^{\prime}, N>0$ such that that

$$
\begin{aligned}
\mid z_{\alpha_{n}^{k_{n}} \beta_{n} \alpha_{n}^{l_{n},+}} & -\frac{a_{n}}{c_{n}} \lambda_{n}^{2 k_{n}} \mid \\
& =\left|\frac{\left(a_{n} \lambda_{n}^{k_{n}+l_{n}}-d_{n} \lambda_{n}^{-l_{n}-k_{n}}\right)+\sqrt{\operatorname{tr}^{2}\left(\alpha_{n}^{k_{n}} \beta_{n} \alpha_{n}^{l_{n}}\right)-4}}{2 c_{n} \lambda_{n}^{l_{n}-k_{n}}}-\frac{a_{n} \lambda_{n}^{k_{n}+l_{n}}}{c_{n} \lambda_{n}^{l_{n}-k_{n}}}\right| \\
& =\left|\frac{\sqrt{\operatorname{tr}^{2}\left(\alpha_{n}^{k_{n}} \beta_{n} \alpha_{n}^{l_{n}}\right)-4}-\sqrt{\operatorname{tr}^{2}\left(\alpha_{n}^{k_{n}} \beta_{n} \alpha_{n}^{l_{n}}\right)}}{2 c_{n} \lambda_{n}^{l_{n}-k_{n}}}\right| \\
& \leq \frac{c}{\left|\lambda_{n}^{l_{n}-k_{n}}\right|\left|\operatorname{tr}\left(\beta_{n}\right)\right|}\left|\sqrt{\operatorname{tr}^{2}\left(\alpha_{n}^{k_{n}} \beta_{n} \alpha_{n}^{l_{n}}\right)-4}-\sqrt{\operatorname{tr}^{2}\left(\alpha_{n}^{k_{n}} \beta_{n} \alpha_{n}^{l_{n}}\right)}\right|
\end{aligned}
$$

If $\left|\operatorname{tr}\left(\alpha_{n}^{k_{n}} \beta_{n} \alpha_{n}^{l_{n}}\right)\right|<\kappa$, for some $\kappa>0$, and all $n$ then this is at most

$$
\frac{c \kappa^{\prime}}{\left|\lambda_{n}^{l_{n}-k_{n}}\right|\left|\operatorname{tr}\left(\beta_{n}\right)\right|} \text { for some } \kappa^{\prime}>0
$$

Otherwise it is at most

$$
c \frac{\left|\sqrt{\operatorname{tr}^{2}\left(\alpha_{n}^{k_{n}} \beta_{n} \alpha_{n}^{l_{n}}\right)-4}\right|}{\left|\lambda_{n}^{l_{n}-k_{n}}\right|\left|\operatorname{tr}\left(\beta_{n}\right)\right|}\left|\frac{1}{\sqrt{1-4 / \operatorname{tr}^{2}\left(\alpha_{n}^{k_{n}} \beta_{n} \alpha_{n}^{l_{n}}\right)}}-1\right| .
$$

Using the Binomial series

$$
\left(1-4 / \operatorname{tr}^{2}\left(\alpha_{n}^{k_{n}} \beta_{n} \alpha_{n}^{l_{n}}\right)\right)^{-1 / 2}=1+2 \operatorname{tr}^{-2}\left(\alpha_{n}^{k_{n}} \beta_{n} \alpha_{n}^{l_{n}}\right)+\epsilon_{n},
$$

with $\epsilon_{n} \rightarrow 0$ at order $\left|\operatorname{tr}^{-4}\left(\alpha_{n}^{k_{n}} \beta_{n} \alpha_{n}^{l_{n}}\right)\right|$ gives $\sigma>0$ such that

$$
\leq \frac{c \sigma}{\left|\lambda_{n}^{l_{n}-k_{n}}\right|\left|\operatorname{tr}\left(\beta_{n}\right)\right|\left|\operatorname{tr}\left(\alpha_{n}^{k_{n}} \beta_{n} \alpha_{n}^{l_{n}}\right)\right|} \quad \text { for large } n .
$$

Hence in either case we have for some $c^{\prime}>0$ that

$$
\leq \frac{c^{\prime}}{\left|\lambda_{n}^{l_{n}-k_{n}}\right|\left|\operatorname{tr}\left(\beta_{n}\right)\right|} \quad \text { for } n>N .
$$

This gives (i) of the Lemma. 
Now $T_{\alpha_{n} k_{n}}, T_{\alpha_{n} l_{n}}<M$ implies $\left|\lambda_{n}^{l_{n}-k_{n}}\right|<M^{\prime}$ for $M^{\prime}>0$. Hence,

$$
\left|z_{\alpha_{n}^{k_{n}} \beta_{n} \alpha_{n}^{l_{n}},+}-\frac{a_{n}}{c_{n}} \lambda_{n}^{2 k_{n}}\right| \leq \frac{c^{\prime \prime}}{\left|\operatorname{tr}\left(\beta_{n}\right)\right|}, \quad n>N, c^{\prime \prime}>0 .
$$

By $4 \cosh \left(T_{\beta_{n}}\right)=\left|\operatorname{tr}^{2}\left(\beta_{n}\right)\right|+\left|\operatorname{tr}^{2}\left(\beta_{n}\right)-4\right|$, we have

$$
e^{T_{\beta_{n}}}<\left|\operatorname{tr}^{2}\left(\beta_{n}\right)\right|\left(1+2 /\left|\operatorname{tr}^{2}\left(\beta_{n}\right)\right|\right) .
$$

Since $\left|\operatorname{tr}\left(\beta_{n}\right)\right| \rightarrow \infty$, there exists $a>0$ such that $e^{T_{\beta_{n}}} \leq a\left|\operatorname{tr}\left(\beta_{n}\right)\right|^{2}$ for sufficiently large $n$.

Note that if $\left|\operatorname{tr}\left(\alpha_{n}^{k_{n}} \beta_{n} \alpha_{n}^{l_{n}}\right)\right| \rightarrow \infty$ then we also have the stronger inequality

$$
\left|z_{\alpha_{n}^{k n} \beta_{n} \alpha_{n}^{l_{n}},+}-\frac{a_{n}}{c_{n}} \lambda_{n}^{2 k_{n}}\right| \leq \frac{c^{\prime \prime \prime}}{\left|\operatorname{tr}\left(\beta_{n}\right)\right|\left|\operatorname{tr}\left(\alpha_{n}^{k_{n}} \beta_{n} \alpha_{n}^{l_{n}}\right)\right|}, \quad n>N, c^{\prime \prime \prime}>0 .
$$

Here we can take $c^{\prime \prime \prime}>c \sigma /\left|\lambda_{n}^{l_{n}-k_{n}}\right|$ as given in the above binomial inequality.

And there exists $b>0$ such that $e^{T_{\alpha_{n}}^{k_{n}} \beta_{n} \alpha_{n}^{l_{n}}} \leq b\left|\operatorname{tr}\left(\alpha_{n}^{k_{n}} \beta_{n} \alpha_{n}^{l_{n}}\right)\right|^{2}$. We therefore need

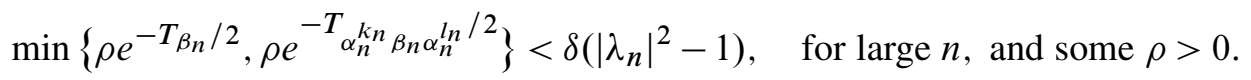
By $\left|\lambda_{n}\right|^{2}=e^{T_{\alpha_{n}}}$, we require

$$
\max \left\{T_{\beta_{n}}, T_{\left.\alpha_{n}^{k_{n}} \beta_{n} \alpha_{n}^{l_{n}}\right\}} \geq 2 \log \left(\frac{\rho / \delta}{e^{T_{\alpha_{n}}-1}}\right) .\right.
$$

Hence it follows there exists $\rho^{\prime}>0$ such that if $D_{n}<\rho^{\prime}$ then at least one of $T_{\beta_{n}}, T_{\alpha_{n}^{k n} \beta_{n} \alpha_{n}^{l n}}$ satisfies the above inequality. Therefore we have

$$
\left|z_{\beta_{n},+}-a_{n} / c_{n}\right|<\delta\left(\left|\lambda_{n}\right|^{2}-1\right)
$$

for $D_{n}<\rho$. The proof for the other part is same.

The case

$$
-\sqrt{\operatorname{tr}^{2}\left(\alpha_{n}^{k_{n}} \beta_{n} \alpha_{n}^{l_{n}}\right)}=\operatorname{tr}\left(\alpha_{n}^{k_{n}} \beta_{n} \alpha_{n}^{l_{n}}\right)
$$

is similar with $z_{\alpha_{n}^{k_{n}} \beta_{n} \alpha_{n}^{l_{n}},+}$ replaced by $z_{\alpha_{n}^{k n} \beta_{n} \alpha_{n}^{l_{n}},-}$ and vice versa.

Remark 4.5 Based on the proof above, we note that the condition $Z_{\left\langle\alpha_{n}, \beta_{n}\right\rangle}>\Delta$ in Lemma 4.2 can be replaced with conditions $\left|\operatorname{tr}\left(\beta_{n}\right)\right| \rightarrow \infty$ and $\left|\operatorname{tr}\left(\beta_{n}\right)\right| \leq\left|c_{n}\right|$. 
Corollary 4.6 Suppose there exists $\Delta>0, M>0$ such that $Z_{\left\langle\alpha_{n}, \beta_{n}\right\rangle}>\Delta$ and $T_{\alpha_{n}}<M$ for all $n$. Let $k_{n}, l_{n}$ to be any integers such that $T_{\alpha_{n} k_{n}}, T_{\alpha_{n} l_{n}}<M$. Then there exist constants $\delta, \rho>0$ such that if $\left|\operatorname{tr}\left(\alpha_{n}^{k_{n}} \beta_{n} \alpha_{n}^{l_{n}}\right)\right| \rightarrow \infty$ then

$$
\left|z_{\alpha_{n}^{k n} \beta_{n} \alpha_{n}^{l_{n}}, \pm}-\zeta_{\alpha_{n}^{k_{n}} \beta_{n} \alpha_{n}^{l_{n}}}\right|+\left|z_{\alpha_{n}^{k_{n}} \beta_{n} \alpha_{n}^{l_{n}}, \mp}-\eta_{\alpha_{n}^{k_{n}} \beta_{n} \alpha_{n}^{l_{n}}}\right|<\frac{\rho}{\left|\operatorname{tr}\left(\alpha_{n}^{k_{n}} \beta_{n} \alpha_{n}^{l_{n}}\right)\right|\left|\operatorname{tr}\left(\beta_{n}\right)\right|}
$$

for all $D_{n}<\delta$. The same dichotomy decomposition of the inequality holds as given in Remark 4.3.

Proof Let us assume that $\sqrt{\operatorname{tr}^{2}\left(\alpha_{n}^{k_{n}} \beta_{n} \alpha_{n}^{l_{n}}\right)}=\operatorname{tr}\left(\alpha_{n}^{k_{n}} \beta_{n} \alpha_{n}^{l_{n}}\right)$. Using the inequality in the proof of Corollary 4.4 and taking $\sigma^{\prime}>c /\left|\lambda_{n}^{l_{n}-k_{n}}\right|$ with $c$ given in the proof of Corollary 4.4 , we have

$$
\left|z_{\alpha_{n}^{k_{n}} \beta_{n} \alpha_{n}^{l_{n}},+}-\frac{a_{n}}{c_{n}} \lambda_{n}^{2 k_{n}}\right| \leq \sigma^{\prime} \frac{\left|\sqrt{\operatorname{tr}^{2}\left(\alpha_{n}^{k_{n}} \beta_{n} \alpha_{n}^{l_{n}}\right)-4}\right|}{\left|\operatorname{tr}\left(\beta_{n}\right)\right|}\left|\frac{1}{\sqrt{1-4 / \operatorname{tr}^{2}\left(\alpha_{n}^{k_{n}} \beta_{n} \alpha_{n}^{l_{n}}\right)}}-1\right| .
$$

By using the binomial series as in the proof of Corollary 4.4 and taking $\sigma^{\prime \prime}>0$ as $\sigma^{\prime \prime}>\sigma \sigma^{\prime} /\left|\lambda_{n}^{l_{n}-k_{n}}\right|$ where $\sigma$ is the constant given in the proof of Corollary 4.4 we have

$$
\left|z_{\alpha_{n}^{k n} \beta_{n} \alpha_{n}^{l_{n},+}}-\frac{a_{n}}{c_{n}} \lambda_{n}^{2 k_{n}}\right| \leq \frac{\sigma^{\prime \prime}}{\left|\operatorname{tr}\left(\alpha_{n}^{k_{n}} \beta_{n} \alpha_{n}^{l_{n}}\right)\right|\left|\operatorname{tr}\left(\beta_{n}\right)\right|} .
$$

The proof for the other part is same.

The case

$$
-\sqrt{\operatorname{tr}^{2}\left(\alpha_{n}^{k_{n}} \beta_{n} \alpha_{n}^{l_{n}}\right)}=\operatorname{tr}\left(\alpha_{n}^{k_{n}} \beta_{n} \alpha_{n}^{l_{n}}\right)
$$

is similar with $z_{\alpha_{n}^{k n} \beta_{n} \alpha_{n}^{l n},+}$ replaced by $z_{\alpha_{n}^{k n} \beta_{n} \alpha_{n}^{l n},-}$ and vice versa.

Lemma 4.7 Suppose there exists $\Delta>0, M>0$ such that $Z_{\left\langle\alpha_{n}, \beta_{n}\right\rangle}>\Delta$ and $T_{\alpha_{n}}<M$ for all $n$. Let $k_{n}, l_{n}$ to be any integers such that $T_{\alpha_{n}} k_{n}, T_{\alpha_{n} l_{n}}<M$. Then there exists constants $\sigma_{1}, \sigma_{2}>0$ such that

$$
\frac{\sigma_{1}\left|\operatorname{tr}\left(\alpha_{n}^{k_{n}} \beta_{n} \alpha_{n}^{l_{n}}\right)\right|}{\left|\operatorname{tr}\left(\beta_{n}\right)\right|} \geq\left|z_{\alpha_{n}^{k_{n}} \beta_{n} \alpha_{n}^{l_{n},+}}-z_{\alpha_{n}^{k_{n}} \beta_{n} \alpha_{n}^{l n},-}\right| \geq \frac{\sigma_{2}\left|\operatorname{tr}\left(\alpha_{n}^{k_{n}} \beta_{n} \alpha_{n}^{l_{n}}\right)\right|}{\left|\operatorname{tr}\left(\beta_{n}\right)\right|},
$$

for all $n$ sufficiently large.

Proof Note that we have

$$
\left|\frac{a_{n}}{c_{n}} \lambda_{n}^{2 k_{n}}-\left(-\frac{d_{n}}{c_{n}} \lambda_{n}^{-2 l_{n}}\right)\right|=\left|\frac{a_{n} \lambda_{n}^{k_{n}+l_{n}}+d_{n} \lambda_{n}^{-k_{n}-l_{n}}}{c_{n} \lambda_{n}^{l_{n}-k_{n}}}\right|=\left|\frac{\operatorname{tr}\left(\alpha_{n}^{k_{n}} \beta_{n} \alpha_{n}^{l_{n}}\right)}{c_{n} \lambda_{n}^{l_{n}-k_{n}}}\right| .
$$


As in the proof of Lemma 4.2, $Z_{\left\langle\alpha_{n}, \beta_{n}\right\rangle}>\Delta$ implies $c<\sqrt{\operatorname{tr}^{2}\left(\beta_{n}\right)-4} /\left(2 c_{n}\right)<c^{\prime}$ for some $c, c^{\prime}>0$ and $\left|\operatorname{tr}\left(\beta_{n}\right)\right| \rightarrow \infty$ implies $\left|\operatorname{tr}\left(\beta_{n}\right)\right| \asymp c_{n}$ (ie $\kappa c_{n}<\left|\operatorname{tr}\left(\beta_{n}\right)\right|<\kappa^{\prime} c_{n}$ for some $\left.\kappa, \kappa^{\prime}>0\right)$. Since $\left|\lambda^{l_{n}-k_{n}}\right|<M^{\prime}$ we have

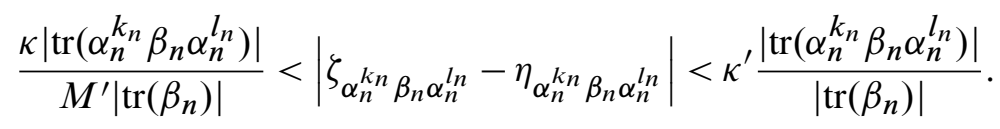

By Lemma 4.2 the result follows.

Although next Lemma is not used in the rest of the paper but we include it here to demonstrate relations between fixed points and Hausdorff dimensions.

Lemma 4.8 Suppose there exists $\Delta>0, M>0$ such that $Z_{\left\langle\alpha_{n}, \beta_{n}\right\rangle}>\Delta$ and $M^{-1}<$ $T_{\alpha_{n}}<M$ for all $n$. Let $k_{n}, l_{n}$ be any integers such that $T_{\alpha_{n} k_{n}}, T_{\alpha_{n} l_{n}}<M$. Then for at least one $i \in\{0,1\}$, and any integers $k_{n}^{\prime}$, lin with $\left|\left(k_{n}-k_{n}^{\prime}\right)\right|+\left|\left(l_{n}-l_{n}^{\prime}\right)\right|=i$, we have

$$
\left|z_{\alpha_{n}^{k_{n}^{\prime}} \beta_{n} \alpha_{n}^{l_{n}^{\prime}},+}-z_{\alpha_{n}^{k_{n}^{\prime}} \beta_{n} \alpha_{n}^{l_{n}^{\prime},-}}\right| \geq \frac{\kappa}{D_{n}\left|\operatorname{tr}\left(\beta_{n}\right)\right|},
$$

for all $n$ sufficiently large.

Proof Since $T_{\alpha_{n}}>M^{-1}$ we have $\lambda_{n} \not \supset 1$. If $\left|\operatorname{tr}\left(\alpha_{n}^{k_{n}} \beta_{n} \alpha_{n}^{l_{n}}\right)\right|<M$ then

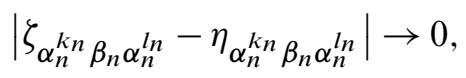

otherwise we have

$$
Z_{\left\langle\alpha_{n}, \alpha_{n}^{k n} \beta_{n} \alpha_{n}^{\left.l_{n}\right\rangle}\right\rangle}>\Delta
$$

for some $\Delta>0$ which implies by Proposition 4.1, $\left|\operatorname{tr}\left(\alpha_{n}^{k_{n}} \beta_{n} \alpha_{n}^{l_{n}}\right)\right| \rightarrow \infty$. Hence we

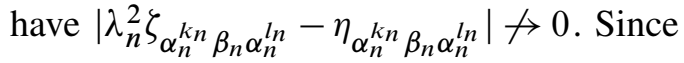

$$
\eta_{\alpha_{n}^{k n+1} \beta_{n} \alpha_{n}^{l_{n}}}=\eta_{\alpha_{n}^{k n} \beta_{n} \alpha_{n}^{l_{n}}} \quad \text { and } \quad \zeta_{\alpha_{n}^{k n+1}} \beta_{n} \alpha_{n}^{l_{n}}=\lambda_{n}^{2} \zeta_{\alpha_{n}^{k n} \beta_{n} \alpha_{n}^{l_{n}}},
$$

by Lemma 4.2 we get

$$
\left|z_{\alpha_{n}^{k_{n}+1} \beta_{n} \alpha_{n}^{l_{n},+}}-z_{\alpha_{n}^{k_{n}+1} \beta_{n} \alpha_{n}^{l_{n},-}}\right|>\kappa .
$$

Then by Lemma 4.7, $\left|\operatorname{tr}\left(\alpha_{n}^{k_{n}+1} \beta_{n} \alpha_{n}^{l_{n}}\right)\right|>\kappa^{\prime}\left|\operatorname{tr}\left(\beta_{n}\right)\right|$. Since $\left|\operatorname{tr}\left(\beta_{n}\right)\right|>\log 3 / D_{n}$ we have $\left|\operatorname{tr}\left(\alpha_{n}^{k_{n}+1} \beta_{n} \alpha_{n}^{l_{n}}\right)\right|>\kappa^{\prime} \log 3 / D_{n}$. Similarly we also have $\left|\operatorname{tr}\left(\alpha_{n}^{k_{n}} \beta_{n} \alpha_{n}^{l_{n}+1}\right)\right|>$ $\kappa^{\prime \prime} \log 3 / D_{n}$. The result follows from Lemma 4.7.

We note that the condition $T_{\alpha_{n}}>M^{-1}$ is of convenience only not necessary, the lemma still holds without this condition. 
Finally in this section we make the following observation based the proof of Lemma 4.2. Note that if we don't care about the precise upper bound of fixed points of $\alpha_{n}^{k_{n}} \beta_{n} \alpha_{n}^{l_{n}}$ to $\zeta_{\alpha_{n}^{k n} \beta_{n} \alpha_{n}^{l n}}, \eta_{\alpha_{n}^{k n} \beta_{n} \alpha_{n}^{l_{n}}}$, then we can relax the conditions in Lemma 4.2 and state as follows:

Remark 4.2.B Suppose that $\left|z_{\beta_{n},+}-z_{\beta_{n},-}\right|<c$ and $\left|c_{n} \lambda_{n}^{l_{n}-k_{n}}\right| \rightarrow \infty$. Then

$$
\left\{z_{\alpha_{n}^{k_{n}} \beta_{n} \alpha_{n}^{l_{n}},+}, z_{\alpha_{n}^{k_{n}} \beta_{n} \alpha_{n}^{l_{n},-}}\right\} \rightarrow\left\{\zeta_{\left.\alpha_{n}^{k_{n}} \beta_{n} \alpha_{n}^{l_{n}}, \eta_{\alpha_{n}^{k_{n}} \beta_{n} \alpha_{n}^{l_{n}}}\right\}}\right.
$$

\section{Sufficient conditions}

In this section we will state and prove a set of conditions for a given sequence of Schottky groups with decreasing Hausdorff dimensions that will be sufficient for the sequence to contain a subsequence of classical Schottky groups.

Let $\left(\mathbb{B}\right.$, dist $\left._{B}\right)$ be the unit ball model of hyperbolic 3 -space. Let $\pi: \mathbb{B} \longrightarrow \mathbb{H}^{3}$ be the stereographic hyperbolic isometry.

Given a loxodromic element $\alpha$, of $\operatorname{PSL}(2, \mathbb{C})$ acting on the unit ball $\mathbb{B}$ model of hyperbolic 3-space, denote by $S_{\alpha, r}$ and $S_{\alpha^{-1}, r}$ the isometric spheres of Euclidean radius $r$ of $\alpha$. We set $\lambda_{\pi_{*}(\alpha)}$ as the multiplier of $\pi_{*}(\alpha)$ in the upper space model $\mathbb{H}^{3}$. For $R>0$, set $C_{R}$ as the circle in $\mathbb{C}$ about origin of radius $R$.

Proposition 5.1 Let $\alpha$ be a loxodromic element of $\operatorname{PSL}(2, \mathbb{C})$ acting on $\mathbb{B}$, with axis passing through the origin and fixed points on north and south poles. Then $\pi\left(S_{\alpha, r} \cap \partial \mathbb{B}\right), \pi\left(S_{\alpha^{-1}, r} \cap \partial \mathbb{B}\right)$ maps to $C_{1 / \lambda_{\pi_{*}(\alpha)}}, C_{\lambda_{\pi_{*}(\alpha)}}$.

Proof Let $T_{\alpha}$ be the translation length of $\alpha$. The Euclidean radius $r$ is given by $r^{-1}=\sinh \left(T_{\alpha} / 2\right)$. In terms of $\lambda_{\pi_{*}(\alpha)}$,

$$
r=\frac{2\left|\lambda_{\pi_{*}(\alpha)}\right|}{\left|\lambda_{\pi_{*}(\alpha)}\right|^{2}-1} .
$$

Set $\mathbf{e}=(0,0,1)$ as the north pole of $\partial \mathbb{B}$. Let $\delta_{\alpha}, \delta_{\alpha^{-1}}>0$ denote the radius of $\pi\left(S_{\alpha, r} \cap \partial \mathbb{B}\right)$ and $\pi\left(S_{\alpha^{-1}, r} \cap \partial \mathbb{B}\right)$ respectively. Then for $x \in S_{\alpha, r} \cap \partial \mathbb{B}, \delta_{\alpha}$ is given by

$$
\delta_{\alpha}^{2}=\frac{4 r^{2}}{\left(1+r^{2}\right)|x-\mathbf{e}|^{4}} \quad \text { where }|x-\mathbf{e}|^{2}=\frac{4}{\left|\lambda_{\pi_{*}(\alpha)}\right|^{2}+1}
$$

which implies

$$
\delta_{\alpha}=\left|\lambda_{\pi_{*}(\alpha)}\right| \text {. }
$$


Similarly for $x \in S_{\alpha^{-1}, r} \cap \partial \mathbb{B}$ we have

$$
\delta_{\alpha^{-1}}^{2}=\frac{4 r^{2}}{\left(1+r^{2}\right)|x-\mathbf{e}|^{4}} \quad \text { where }|x-\mathbf{e}|^{2}=\frac{4\left|\lambda_{\pi_{*}(\alpha)}\right|^{2}}{\left|\lambda_{\pi_{*}(\alpha)}\right|^{2}+1}
$$

which gives

$$
\delta_{\alpha^{-1}}=\frac{1}{\left|\lambda_{\pi_{*}(\alpha)}\right|} .
$$

This completes the proof.

Lemma 5.2 Let $\left\langle\alpha_{n}, \beta_{n}\right\rangle$ be generators for Schottky groups $\Gamma_{n}$ in the upper-half space model $\mathbb{H}^{3}$ with $\left|\operatorname{tr}\left(\beta_{n}\right)\right| \rightarrow \infty$ and

$$
\alpha_{n}=\left(\begin{array}{cc}
\lambda_{n} & 0 \\
0 & \lambda_{n}^{-1}
\end{array}\right), \quad\left|\lambda_{n}\right|>1 .
$$

Suppose one of the following set of conditions holds:

- There exists $\Lambda>1$ such that for large $n$, we have $\left|\lambda_{n}\right|<\Lambda$ and

$$
\left|\lambda_{n}\right|^{-1}<\left|z_{\beta_{n}, l}\right| \leq\left|z_{\beta_{n}, u}\right|<\mid \lambda_{n},
$$

$$
\liminf _{n}\left\{\frac{1}{\left(\left|z_{\beta_{n}, u}\right|-\left|\lambda_{n}\right|\right)\left|\operatorname{tr}\left(\beta_{n}\right)\right|}, \frac{1}{\left(\left|z_{\beta_{n}, l}\right|-\left|\lambda_{n}\right|^{-1}\right)\left|\operatorname{tr}\left(\beta_{n}\right)\right|}\right\}=0 .
$$

- There exists $\kappa>0$ and for large $n$, we have $\left|\lambda_{n}\right|>\kappa$ and

$$
\kappa^{-1}<\left|z_{\beta_{n}, l}\right| \leq\left|z_{\beta_{n}, u}\right|<\kappa,
$$

$$
\liminf _{n}\left\{\frac{1}{\left(\left|z_{\beta_{n}, u}\right|-\kappa\right)\left|\operatorname{tr}\left(\beta_{n}\right)\right|}, \frac{1}{\left(\left|z_{\beta_{n}, l}\right|-\kappa^{-1}\right)\left|\operatorname{tr}\left(\beta_{n}\right)\right|}\right\}=0 \text {. }
$$

Then there exists a subsequence such that for $i$ large, $\pi^{-1}\left\langle\alpha_{n_{i}}, \beta_{n_{i}}\right\rangle \pi$ are classical generators for $\Gamma_{n_{i}}$ in the unit ball model $\mathbb{B}$.

Proof Let us suppose there exists a subsequence $\left\langle\alpha_{n_{i}}, \beta_{n_{i}}\right\rangle$ that satisfies the first set of conditions. First assume that for large $i,\left|z_{\beta_{n_{i}, u}}-z_{\beta_{n_{i}, l}}\right|>\delta>0$.

Let $r_{i}, \rho_{i}$ denote the Euclidean radii of the isometric spheres of $\pi^{-1} \alpha_{n_{i}} \pi, \pi^{-1} \beta_{n_{i}} \pi$ respectively. Note that $4 \cosh \left(T_{\pi^{-1} \beta_{n_{i}} \pi}\right) \geq\left|\operatorname{tr}^{2}\left(\pi^{-1} \beta_{n_{i}} \pi\right)\right|$, which implies there exists $c^{\prime}>0$ such that

$$
e^{T_{\pi-1} \beta_{n_{i}} \pi} \geq c^{\prime}\left|\operatorname{tr}^{2}\left(\pi^{-1} \beta_{n_{i}} \pi\right)\right| .
$$

Since $\rho_{i}^{-1}=\cosh \operatorname{dist}\left(o, \mathcal{L}_{\pi^{-1} \beta_{n_{i}} \pi}\right) \sinh \left(\frac{1}{2} T_{\pi^{-1}} \beta_{n_{i}} \pi\right)$ [2, page 175], we have $\rho_{i}^{-1} \geq$ $\sinh \left(\frac{1}{2} T_{\pi^{-1} \beta_{n_{i}} \pi}\right)$, so for large $i$ there exists $c>0$ such that

$$
\rho_{i}^{-1} \geq c e^{(1 / 2) T_{\pi-1} \beta_{n_{i}} \pi} .
$$


Hence there exists $\delta_{1}>0$ such that $\rho_{i} \leq \delta_{1}\left|\operatorname{tr}\left(\beta_{n_{i}}\right)\right|^{-1}$ for large $i$. Since for $z, w \in \mathbb{C}$,

and

$$
\begin{aligned}
\left|\pi^{-1}(z)^{\prime}\right| & =\frac{2}{|z+\mathbf{e}|^{2}} \\
\left|\pi^{-1}(z)-\pi^{-1}(w)\right| & =\left|\pi^{-1}(z)^{\prime}\right|^{1 / 2}\left|\pi^{-1}(w)^{\prime}\right|^{1 / 2}|z-w| .
\end{aligned}
$$

This implies for large $i$, and $x_{i} \in C_{\left|z_{\beta n_{i}, u}\right|}$ and $y_{i} \in C_{\left|\lambda_{n_{i}}\right|}$,

$$
\frac{\rho_{i}}{\left|\pi^{-1} x_{i}-\pi^{-1} y_{i}\right|} \leq \frac{2 \delta_{1}\left|x_{i}+\mathbf{e}\right|\left|y_{i}+\mathbf{e}\right|}{\left|\operatorname{tr}\left(\beta_{n_{i}}\right)\right|\left|x_{i}-y_{i}\right|} .
$$

Since $\left|\lambda_{n_{i}}\right|<\Lambda$, there exists $\delta_{2}>0$ such that $\left|x_{i}+\mathbf{e}\right|\left|y_{i}+\mathbf{e}\right|<\delta_{2}$, and

$$
\lim _{i} \frac{\rho_{i}}{\left|\pi^{-1} x_{i}-\pi^{-1} y_{i}\right|} \leq \lim _{i} \frac{2 \delta_{1} \delta_{2}}{\left|\operatorname{tr}\left(\beta_{n_{i}}\right)\right|\left|x_{i}-y_{i}\right|}=0 .
$$

Similarly there exists $\delta_{3}>0$ such that for $w_{i} \in C_{\left|z_{\beta n_{i}, l}\right|}$ and $z_{i} \in C_{\left|\lambda_{n_{i}}\right|^{-1}}$,

$$
\lim _{i} \frac{\rho_{i}}{\left|\pi^{-1} w_{i}-\pi^{-1} z_{i}\right|} \leq \lim _{i} \frac{2 \delta_{1} \delta_{3}}{\left|\operatorname{tr}\left(\beta_{n_{i}}\right)\right|\left|w_{i}-z_{i}\right|}=0 .
$$

Hence it follows that for large $i$, and Proposition 5.1, the isometric spheres $S_{\pi^{-1}} \alpha_{n_{i}} \pi, r_{i}$, $S_{\pi^{-1} \alpha_{n_{i}}^{-1} \pi, r_{i}}, S_{\pi^{-1} \beta_{n_{i}} \pi, \rho_{i}}, S_{\pi^{-1} \beta_{n_{i}}^{-1} \pi, \rho_{i}}$ are disjoint.

Remark 5.3 Note that if we don't assume that $\left|\lambda_{n_{i}}\right|<\Lambda$ then we don't have bounds on $\left|x_{i}+\mathbf{e}\right|\left|y_{i}+\mathbf{e}\right|$. However, since $\left|x_{i}+\mathbf{e}\right|\left|y_{i}+\mathbf{e}\right| \leq\left(\left|z_{\beta_{n_{i}}, u}\right|+1\right)\left(\left|\lambda_{n_{i}}\right|+1\right)$ for $x_{i} \in C_{\left|z_{\beta_{n_{i}}, u}\right|}, y_{i} \in C_{\left|\lambda_{n_{i}}\right|}$ and $\left|x_{i}+\mathbf{e}\right|\left|y_{i}+\mathbf{e}\right| \leq\left(\left|z_{\beta_{n_{i}}, l}\right|+1\right)\left(\left|\lambda_{n_{i}}\right|^{-1}+1\right)$ for $x_{i} \in C_{\left|z_{\beta_{i}, l}\right|}, y_{i} \in C_{\left|\lambda_{n_{i}}\right|} \mid-1$. Hence we can state the condition as follows:

$$
\begin{gathered}
\left|\lambda_{n}\right|^{-1}<\left|z_{\beta_{n}, l}\right| \leq\left|z_{\beta_{n}, u}\right|<\left|\lambda_{n}\right|, \\
\liminf _{n}\left\{\frac{\left(\left|z_{\beta_{n}, u}\right|+1\right)\left(\left|\lambda_{n}\right|+1\right)}{\left(\left|z_{\beta_{n}, u}\right|-\left|\lambda_{n}\right|\right)\left|\operatorname{tr}\left(\beta_{n}\right)\right|}, \frac{\left(\left|z_{\beta_{n}, l}\right|+1\right)\left(\left|\lambda_{n}\right|^{-1}+1\right)}{\left(\left|z_{\beta_{n}, l}\right|-\left|\lambda_{n}\right|^{-1}\right)\left|\operatorname{tr}\left(\beta_{n}\right)\right|}\right\}=0 .
\end{gathered}
$$

Next let us assume that $\left|z_{\beta_{n_{i}}, u}-z_{\beta_{n_{i}}, l}\right| \rightarrow 0$. Under this assumption we can do a much stronger estimate of the lower bounds of $\cosh \operatorname{dist}\left(j, \mathcal{L}_{\beta_{n_{i}}}\right)$, distance between the point $j$ on the vertical $j$-axis and the axis of $\beta_{n_{i}}$ in the $\mathbb{H}^{3}$. Note however that a weaker lower bounds is sufficient in our case.

Recall that given any two points $h_{1}=\left(z_{1}, \theta_{1}\right), h_{2}=\left(z_{2}, \theta_{2}\right) \in \mathbb{H}^{3}$ the hyperbolic distance is given by

$$
\cosh \operatorname{dist}\left(h_{1}, h_{2}\right)=\frac{\left|z_{1}-z_{2}\right|^{2}+\left|\theta_{1}-\theta_{2}\right|^{2}}{2 \theta_{1} \theta_{2}}+1 .
$$


Since $\left|z_{\beta_{n_{i}}, u}-z_{\beta_{n_{i}}, l}\right| \rightarrow 0$ and $1 / \Lambda<\left|z_{\beta_{n_{i}, l}}\right| \leq\left|z_{\beta_{n_{i}}, u}\right|<\Lambda$ we can estimate the quantity cosh dist $\left(j, \mathcal{L}_{\beta_{n_{i}}}\right)$ by using the above formula for $\left(z_{1}, \theta_{1}\right) \in \mathcal{L}_{\alpha_{n_{i}}}$ and $\left(z_{2}, \theta_{2}\right) \in$ $\mathcal{L}_{\beta_{n_{i}}}$. Since for large $i$ we have $\left|z_{1}-z_{2}\right| \geq\left|z_{\beta_{n_{i}}, l}\right|$,

$$
\left|\theta_{1}-\theta_{2}\right| \geq|| z_{\beta_{n_{i}}, l}\left|-\frac{1}{2}\left(\left|z_{\beta_{n_{i}}, u}-z_{\beta_{n_{i}}, l}\right|\right)\right|, \quad 2 \theta_{1} \theta_{2} \leq\left|z_{\beta_{n_{i}}, u}\right|\left|z_{\beta_{n_{i}}, u}-z_{\beta_{n_{i}}, l}\right| .
$$

Hence for large $i$ we have

$$
\cosh \operatorname{dist}\left(j, \mathcal{L}_{\beta_{n_{i}}}\right) \geq \frac{\left|z_{\beta_{n_{i}}, l}\right|^{2}+\left(\left|z_{\beta_{n_{i}}, l}\right|-\frac{1}{2}\left(\left|z_{\beta_{n_{i}}, u}-z_{\beta_{n_{i}}, l}\right|\right)\right)^{2}}{\left|z_{\beta_{n_{i}}, u}\right|\left|z_{\beta_{n_{i}}, u}-z_{\beta_{n_{i}}, l}\right|}+1 .
$$

Since $|\Lambda|^{-1}<\left|z_{\beta_{n_{i}}, l}\right| \leq\left|z_{\beta_{n_{i}}, u}\right|<\Lambda$ and $\left|z_{\beta_{n_{i}}, u}-z_{\beta_{n_{i}}, l}\right| \rightarrow 0$, we have for large $i$ there exists $\sigma>0$ such that

$$
\cosh \operatorname{dist}\left(j, \mathcal{L}_{\beta_{n_{i}}}\right) \geq \frac{\sigma}{\left|z_{\beta_{n_{i}}, u}-z_{\beta_{n_{i}}, l}\right|} .
$$

Also by $\Lambda^{-1}<\left|z_{\beta_{n_{i}}, l}\right| \leq\left|z_{\beta_{n_{i}}, u}\right|<\Lambda$ and $\left|\lambda_{n_{i}}\right|<|\Lambda|$, there exists $\sigma^{\prime}>0$ such that $\left|\pi^{-1}\left(z_{\beta_{n_{i}}, u}\right)^{\prime}\right|\left|\pi^{-1}\left(z_{\beta_{n_{i}}, l}\right)^{\prime}\right|>\sigma^{\prime}$. Since

$$
\left|\pi^{-1} z_{\beta_{n_{i}}, u}-\pi^{-1} z_{\beta_{n_{i}}, l}\right|=\left|\pi^{-1}\left(z_{\beta_{n_{i}}, u}\right)^{\prime}\right|\left|\pi^{-1}\left(z_{\beta_{n_{i}}, l}\right)^{\prime}\right|\left|z_{\beta_{n_{i}}, u}-z_{\beta_{n_{i}}, l}\right|,
$$

we have $\left|\pi^{-1} z_{\beta_{n_{i}}, u}-\pi^{-1} z_{\beta_{n_{i}}, l}\right| \geq \sigma^{\prime}\left|z_{\beta_{n_{i}}, u}-z_{\beta_{n_{i}}, l}\right|$,

The equality $\rho_{i}^{-1}=\cosh \operatorname{dist}\left(o, \mathcal{L}_{\pi^{-1}} \beta_{n_{i}} \pi\right) \sinh \left(\frac{1}{2} T_{\pi^{-1}} \beta_{n_{i}} \pi\right)$ and the above estimates imply that for $i$ large, there exists $\delta_{4}>0$ such that

$$
\rho_{i} \leq \delta_{4}\left|\pi^{-1} z_{\beta_{n_{i}}, u}-\pi^{-1} z_{\beta_{n_{i}}, l}\right|\left|\operatorname{tr}\left(\beta_{n_{i}}\right)\right|^{-1} .
$$

Hence there exists $\delta_{5}>0$ such that for $x_{i} \in C_{\left|z_{\beta_{n_{i}}, u}\right|}, y_{i} \in C_{\left|\lambda_{n_{i}}\right|}$,

$$
\lim _{i} \frac{\rho_{i}}{\left|\pi^{-1} x_{i}-\pi^{-1} y_{i}\right|} \leq \lim _{i} \frac{\delta_{5}\left|\pi^{-1} z_{\beta_{n_{i}}, u}-\pi^{-1} z_{\beta_{n_{i}}, l}\right|}{\left|\operatorname{tr}\left(\beta_{n_{i}}\right)\right|\left|x_{i}-y_{i}\right|}=0 .
$$

Similarly there exists $\delta_{6}>0$ such that for $w_{i} \in C_{\left|z_{\beta n_{i}, l}\right|}$ and $z_{i} \in C_{\left|\lambda_{n_{i}}\right|^{-1}}$,

$$
\lim _{i} \frac{\rho_{i}}{\left|\pi^{-1} w_{i}-\pi^{-1} z_{i}\right|} \leq \lim _{i} \frac{\delta_{6}\left|\pi^{-1} z_{\beta_{n_{i}, u}}-\pi^{-1} z_{\beta_{n_{i}, l} \mid}\right|}{\left|\operatorname{tr}\left(\beta_{n_{i}}\right)\right|\left|w_{i}-z_{i}\right|}=0 .
$$

From these estimates and Proposition 5.1 we have for sufficiently large $i, S_{\pi^{-1} \alpha_{n_{i}} \pi, r_{i}}$, $S_{\pi^{-1} \alpha_{n_{i}}^{-1} \pi, r_{i}}$ are disjoint from $S_{\pi^{-1} \beta_{n_{i} \pi, \rho_{i}}, S_{\pi^{-1}} \beta_{n_{i}}^{-1} \pi, \rho_{i}}$. Since $\left|\operatorname{tr}\left(\beta_{n_{i}}\right)\right| \rightarrow \infty$ implies $S_{\pi^{-1} \beta_{n_{i} \pi, \rho_{i}}}$ and $S_{\pi^{-1} \beta_{n_{i}}^{-1} \pi, \rho_{i}}$ are disjoint when $i$ is large, we have the first part of the lemma.

The second part of the lemma can be proved in the same way. 
Remark 5.4 Note that in the course of the proof we see that if $\left|\lambda_{n}\right| \rightarrow 1$ then we can weaken the first set of conditions in the above lemma to

$$
\begin{gathered}
\left|\lambda_{n}\right|^{-1}<\left|z_{\beta_{n}, l}\right| \leq\left|z_{\beta_{n}, u}\right|<\left|\lambda_{n}\right|, \\
\liminf _{n}\left\{\frac{\left|z_{\beta_{n}, l}-z_{\beta_{n}, u}\right|}{\left(\left|z_{\beta_{n}, u}\right|-\left|\lambda_{n}\right|\right)\left|\operatorname{tr}\left(\beta_{n}\right)\right|}, \frac{\left|z_{\beta_{n}, l}-z_{\beta_{n}, u}\right|}{\left(\left|z_{\beta_{n}, l}\right|-\left|\lambda_{n}\right|^{-1}\right)\left|\operatorname{tr}\left(\beta_{n}\right)\right|}\right\}=0,
\end{gathered}
$$

\section{Schottky subspaces $\mathfrak{J}_{k}(\tau)$}

This section is devoted to proving Theorem 6.1 by utilizing results established in the previous sections.

For $\tau>0$, define $\mathfrak{J}_{k}(\tau):=\left\{\Gamma \in \mathfrak{J}_{k} \mid Z_{\Gamma}>\tau\right\}$. Recall that $\mathfrak{J}_{k}$ denotes set of all Schottky groups of rank $k$.

Theorem 6.1 Let $\mathfrak{J}_{2}$ be the set of all 2-generated Schottky groups. For each $\tau>0$ there exists a $v>0$ such that $\left\{\Gamma \in \mathfrak{J}_{2}(\tau) \mid D_{\Gamma} \leq v\right\} \subset \mathfrak{J}_{k, o}$.

Proof We prove by contradiction. Assume there exists a sequence $\left\{\Gamma_{n}\right\} \subset \mathfrak{J}_{2}(\tau)$ of nonclassical Schottky groups with $D_{n} \rightarrow 0$. By passing to subsequence, we may assume $D_{n} \rightarrow 0$ monotonically. Set $\Gamma_{n}=\left\langle\alpha_{n}, \beta_{n}\right\rangle$ with $Z_{\left\langle\alpha_{n}, \beta_{n}\right\rangle}>\tau$. We arrange the generators so that $\left|\operatorname{tr}\left(\alpha_{n}\right)\right| \leq\left|\operatorname{tr}\left(\beta_{n}\right)\right|$. There are two possibilities: (I) There exists a subsequence such that $\left|\operatorname{tr}\left(\alpha_{n_{i}}\right)\right| \rightarrow \infty$, and (II) $\left|\operatorname{tr}\left(\alpha_{n}\right)\right|<M$, for some $M>0$.

Case (I) is trivial. Since both $\left|\operatorname{tr}\left(\alpha_{n_{i}}\right)\right|,\left|\operatorname{tr}\left(\beta_{n_{i}}\right)\right| \rightarrow \infty$ as $n \rightarrow \infty$, it follows from $Z_{\left\langle\alpha_{n}, \beta_{n}\right\rangle}>\tau$, there must exists $N$ such that $\left\langle\alpha_{n}, \beta_{n}\right\rangle$ becomes classical Schottky groups for $n>N$. A contradiction.

Now we consider Case (II). We work in upper space model $\mathbb{H}^{3}$. Conjugate $\left\langle\alpha_{n}, \beta_{n}\right\rangle$ by a Mobius transformation into

Denote

$$
\begin{aligned}
\alpha_{n} & =\left(\begin{array}{cc}
\lambda_{n} & 0 \\
0 & \lambda_{n}^{-1}
\end{array}\right), \quad\left|\lambda_{n}\right|>1 . \\
\beta_{n} & =\left(\begin{array}{ll}
a_{n} & b_{n} \\
c_{n} & d_{n}
\end{array}\right) .
\end{aligned}
$$

Since $\left|\operatorname{tr}\left(\alpha_{n}\right)\right|<M$ implies $\left|\lambda_{n}\right|<M^{\prime}$ for some $M^{\prime}>0$, it follows from Proposition 4.1 and $D_{n} \rightarrow 0$, we have $\left|\operatorname{tr}\left(\beta_{n}\right)\right| \rightarrow \infty$. In addition, by conjugation with Mobius transformations, we can assume $\beta_{n}$ have $\eta_{\beta_{n}}=1$. By replacing $\beta_{n}$ with $\beta_{n}^{-1}$ if necessary, we can assume $\left|\zeta_{\beta_{n}}\right| \leq\left|\eta_{\beta_{n}}\right|$. 
Since $Z_{\left\langle\alpha_{n}, \beta_{n}\right\rangle}>\tau$, there exists $\Delta_{1}, \Delta_{2}, \Delta_{3}, \Delta_{4}>0$ such that $\Delta_{1}<\left|z_{\beta_{n}, l}\right| \leq\left|z_{\beta_{n}, u}\right|<$ $\Delta_{2}$, and $\Delta_{3}<\left|z_{\beta_{n}, l}-z_{\beta_{n}, u}\right|<\Delta_{4}$. It follows from Lemma 4.2, $\Delta_{1}<\left|\zeta_{\beta_{n}}\right| \leq\left|\eta_{\beta_{n}}\right|<$ $\Delta_{2}$, and $\Delta_{3}<\lim _{n}\left|\zeta_{\beta_{n}}-\eta_{\beta_{n}}\right|<\Delta_{4}$.

For each $n$, choose integers $k_{n}$ such that

$$
1 \leq\left|\zeta_{\beta_{n}} \lambda_{n}^{2 k_{n}}\right|<\left|\lambda_{n}^{2}\right|
$$

We consider the generating set $\left\langle\alpha_{n}, \alpha_{n}^{k_{n}} \beta_{n}\right\rangle$.

By passing to subsequence if necessary, there are three cases that need to be considered (to simplify the notation, we denote subsequences by the same index notation):
(A) $\left|\zeta_{\beta_{n}} \lambda_{n}^{2 k_{n}}\right| \rightarrow 1$.
(B) $\left.|| \zeta_{\beta_{n}} \lambda_{n}^{2 k_{n}}|-| \lambda_{n}\right|^{2} \mid \rightarrow 0$.
(C) Cases (A) and (B) do not occur.

\subsection{Case (C)}

We use the same notation index for subsequences. Since cases (A) and (B) do not occur, for large $n$ there exists $1<|\lambda|<\left|\lambda_{n}\right|, \sigma<1$ such that $\left|\zeta_{n} \lambda_{n}^{2 k_{n}}\right| \rightarrow \sigma|\lambda|^{2}$. Let $\psi_{n}$ be the Mobius transformation that fixes $\{0, \infty\}$ defined by $\psi(x)=x /(\sqrt{\sigma}|\lambda|), x \in \mathbb{C}$. Then $\left|\eta_{\psi \alpha_{n}^{k n} \beta_{n} \psi^{-1}}\right| \rightarrow 1 /(\sqrt{\sigma}|\lambda|)$ and $\left|\zeta_{\psi \alpha_{n}^{k_{n}} \beta_{n} \psi^{-1}}\right| \rightarrow \sqrt{\sigma}|\lambda|$. By Lemma 4.2, for $n$ large,

$$
\max \left\{\left|z_{\psi \alpha_{n}^{k n} \beta_{n} \psi^{-1}, \mp}-\eta_{\psi \alpha_{n}^{k n} \beta_{n} \psi^{-1}}\right|,\left|z_{\psi \alpha_{n}^{k n} \beta_{n} \psi^{-1}, \pm}-\zeta_{\psi \alpha_{n}^{k n} \beta_{n} \psi^{-1}}\right|\right\}<\frac{\rho}{\left|\operatorname{tr}\left(\beta_{n}\right)\right|} .
$$

Hence there exists $\rho^{\prime}, \rho^{\prime \prime}, \rho^{\prime \prime \prime}>0$ such that

and

$$
\begin{aligned}
& \left|z_{\psi \alpha_{n}^{k n} \beta_{n} \psi^{-1,+}}-z_{\psi \alpha_{n}^{k n} \beta_{n} \psi^{-1,-}}\right|>|| \sqrt{\sigma}|\lambda|-\frac{1}{\sqrt{\sigma}}|\lambda| \mid-\frac{\rho^{\prime}}{\left|\operatorname{tr}\left(\beta_{n}\right)\right|}, \\
& \left|\frac{1}{z_{\psi \alpha_{n}^{k n} \beta_{n} \psi^{-1},+}}-\frac{1}{z_{\psi \alpha_{n}^{k n} \beta_{n} \psi^{-1,-}}}\right|>\rho^{\prime \prime}|| \sqrt{\sigma}|\lambda|-\frac{1}{\sqrt{\sigma}}|\lambda| \mid-\frac{\rho^{\prime \prime \prime}}{\left|\operatorname{tr}\left(\beta_{n}\right)\right|} .
\end{aligned}
$$

This implies that there exist $\Delta>0$ such that

$$
Z_{\psi \alpha_{n}^{k n} \beta_{n} \psi^{-1}}>\Delta
$$

Hence applying Proposition 4.1 to the generators $\left\langle\psi \alpha_{n} \psi^{-1}, \psi \alpha_{n}^{k_{n}} \beta_{n} \psi^{-1}\right\rangle$ implies $\left|\operatorname{tr}\left(\psi \alpha_{n}^{k_{n}} \beta_{n} \psi^{-1}\right)\right| \rightarrow \infty$. 
Set $\kappa=\sqrt{(\sigma+1) / 2}|\lambda|$. Then for sufficiently large $n$ we have $\kappa<\left|\lambda_{n}\right|, \kappa^{-1}<$ $\left|z_{\psi \alpha_{n}^{k n} \beta_{n} \psi^{-1}, l}\right| \leq\left|z_{\psi \alpha_{n}^{k_{n}} \beta_{n} \psi^{-1}, u}\right|<\kappa$. And obviously,

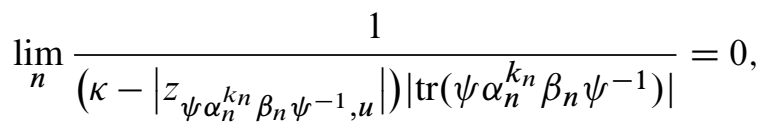

$$
\begin{aligned}
& \lim _{n} \frac{1}{\left(\left|z_{\psi \alpha_{n}^{k n} \beta_{n} \psi^{-1, l}}\right|-\kappa^{-1}\right)\left|\operatorname{tr}\left(\psi \alpha_{n}^{k_{n}} \beta_{n} \psi^{-1}\right)\right|}=0 .
\end{aligned}
$$

Therefore, $\left\langle\psi \alpha_{n} \psi^{-1}, \psi \alpha_{n}^{k_{n}} \beta_{n} \psi^{-1}\right\rangle$ satisfies the second set of conditions of Lemma 5.2, and so by Lemma 5.2, these will be classical generators for large $n$, a contradiction.

\subsection{Case (A)}

By passing to a subsequence if necessary, we have two possibilities:

(A $\left.\mathrm{A}_{1}\right)\left|\lambda_{n}\right|^{2}-1$ is monotonically decreasing to 0 .

$\left(\mathrm{A}_{2}\right)$ There exists $\lambda>1$ such that $\left|\lambda_{n}\right| \geq|\lambda|$ for large $n$.

6.2.1 ( $\left.\mathbf{A}_{1}\right)$ Here we either have

(i) $\left.\lim \sup _{n}|| \zeta_{\alpha_{n}^{k_{n} \beta_{n}}}|-| \lambda_{n}\right|^{2}|| \zeta_{\alpha_{n}^{k_{n}} \beta_{n}}-\left.1\right|^{-1}<\infty$, or

(ii) $\left.\liminf _{n}|| \zeta_{\alpha_{n}^{k n} \beta_{n}}|-| \lambda_{n}\right|^{2}|| \zeta_{\alpha_{n}^{k n} \beta_{n}}-\left.1\right|^{-1} \rightarrow \infty$.

If $\alpha_{n}^{k_{n}} \beta_{n}$ satisfies (ii), we conjugate $\alpha_{n}^{k_{n}} \beta_{n}$ with Mobius transformation $\psi_{n}$ defined by

$$
\psi_{n}(x)=\frac{x}{\zeta_{\alpha_{n}^{k n} \beta_{n}}}
$$

Consider $\left(\psi_{n} \alpha_{n}^{k_{n}} \beta_{n} \alpha_{n}^{l_{n}} \psi_{n}^{-1}\right)^{-1}$ and take $l_{n}=-1$. By factoring out $\zeta_{\alpha_{n}^{k_{n}} \beta_{n}}^{2} \lambda_{n}^{-2}$ in (ii),

$$
\begin{gathered}
\liminf _{n}\left|\zeta_{\alpha_{n}^{k_{n}} \beta_{n}}\right|^{2}\left|\lambda_{n}\right|^{-2}|| 1-\left|\zeta_{\alpha_{n}^{k_{n}} \beta_{n}}\right|^{-1}\left|\lambda_{n}\right|^{2}|| \zeta_{\alpha_{n}^{k n} \beta_{n}}^{-1} \lambda_{n}^{2}-\left.\lambda_{n}^{2}\right|^{-1} \rightarrow \infty \\
\liminf _{n}|| 1-\left|\zeta_{\alpha_{n}^{k_{n}} \beta_{n}}^{-1} \lambda_{n}^{2}\right||| \zeta_{\alpha_{n}^{k_{n}} \beta_{n}}^{-1} \lambda_{n}^{2}-\left.\lambda_{n}^{2}\right|^{-1} \rightarrow \infty
\end{gathered}
$$

Since $\zeta_{\alpha_{n}^{k_{n}} \beta_{n}}^{-1} \lambda_{n}^{2}=\eta_{\alpha_{n}^{k_{n}} \beta_{n} \alpha_{n}^{-1}}$ and $\zeta_{\left(\psi_{n} \alpha_{n}^{k_{n}} \beta_{n} \alpha_{n}^{-1} \psi_{n}^{-1}\right)^{-1}}=\eta_{\psi_{n} \alpha_{n}^{k_{n}} \beta_{n} \alpha_{n}^{-1} \psi_{n}^{-1}}$ we have

$$
\liminf _{n}|| 1-\left|\eta_{\alpha_{n}^{k n} \beta_{n} \alpha_{n}^{-1}}\right|^{2}|| \eta_{\alpha_{n}^{k n} \beta_{n} \alpha_{n}^{-1}}-\left.\lambda_{n}^{2}\right|^{-1} \rightarrow \infty
$$


giving

$\left.\limsup _{n}|| \zeta_{\left(\psi_{n} \alpha_{n}^{k n} \beta_{n} \alpha_{n}^{-1} \psi_{n}^{-1}\right)^{-1}}|-| \lambda_{n}\right|^{2}|| \zeta_{\left(\psi_{n} \alpha_{n}^{k n} \beta_{n} \alpha_{n}^{-1} \psi_{n}^{-1}\right)^{-1}}-\left.1\right|^{-1}<\infty$.

The generator $\left(\psi_{n} \alpha_{n}^{k_{n}} \beta_{n} \alpha_{n}^{-1} \psi_{n}^{-1}\right)^{-1}$ satisfies (i). Hence replacing the generators if necessary we can always assume the generators satisfy (i). And without lost of generality we will assume that $\left\langle\alpha_{n}, \alpha_{n}^{k_{n}} \beta_{n}\right\rangle$ satisfies (i).

Consider (i).

In this case, we have either

(i $\left.\mathrm{i}_{1}\right)\left.\operatorname{lim\operatorname {sup}_{n}}|| \zeta_{\alpha_{n}^{k_{n}} \beta_{n}}|-| \lambda_{n}\right|^{2}|| \zeta_{\alpha_{n}^{k_{n}} \beta_{n}}-\left.1\right|^{-1}>\delta>0$, or

(i $\left.\mathrm{i}_{2}\right)\left.\quad \lim \sup _{n}|| \zeta_{\alpha_{n}^{k_{n}} \beta_{n}}|-| \lambda_{n}\right|^{2}|| \zeta_{\alpha_{n}^{k_{n}} \beta_{n}}-\left.1\right|^{-1}=0$.

Consider ( $\left.i_{1}\right)$.

Lemma 6.2 There exists $c>0$ such that

$$
\operatorname{dist}\left(\mathcal{L}_{\alpha_{n}}, \mathcal{L}_{\alpha_{n}^{k n} \beta_{n}}\right)<\log \left(\frac{c}{\left|\lambda_{n}\right|^{2}-1}\right)
$$

Proof We first show that

$$
\frac{1}{\left|\operatorname{tr}\left(\beta_{n}\right)\right|\left(\left|\lambda_{n}\right|^{2}-1\right)} \rightarrow 0
$$

From Proposition 4.1 we have

$$
\lim \frac{1}{\left|\operatorname{tr}\left(\beta_{n}\right)\right|\left(\left|\lambda_{n}\right|^{2}-1\right)} \leq \lim \rho\left(\frac{\left|\lambda_{n}\right|^{2 D_{n}}-1}{\left(\left|\lambda_{n}\right|^{2 D_{n}}+3\right)\left(\left|\lambda_{n}\right|^{2}-1\right)^{2 D_{n}}}\right)^{1 /\left(2 D_{n}\right)}
$$

and for large $n$, we have $\left|\lambda_{n}\right|^{2 D_{n}}-1<\left|\lambda_{n}\right|^{2}-1$ which implies that for some $\rho^{\prime}>0$,

$$
\lim \frac{1}{\left|\operatorname{tr}\left(\beta_{n}\right)\right|\left(\left|\lambda_{n}\right|^{2}-1\right)} \leq \lim \rho^{\prime}\left(\left|\lambda_{n}\right|^{2}-1\right)^{\left(1-2 D_{n}\right) /\left(2 D_{n}\right)}=0 .
$$

It follows from Lemma 4.2 and $\eta_{\alpha_{n}^{k n} \beta_{n}}=1$ that

$$
\left|\zeta_{\alpha_{n}^{k n} \beta_{n}}-1\right|-\rho^{\prime}\left|\operatorname{tr}\left(\beta_{n}\right)\right|^{-1} \leq\left|z_{\alpha_{n}^{k n} \beta_{n},-}-z_{\alpha_{n}^{k n} \beta_{n},+}\right| \leq\left|\zeta_{\alpha_{n}^{k n} \beta_{n}}-1\right|+\rho^{\prime \prime}\left|\operatorname{tr}\left(\beta_{n}\right)\right|^{-1}
$$

Since $1 \leq\left|\zeta_{\alpha_{n}^{k n} \beta_{n}}\right|<\left|\lambda_{n}\right|^{2}$, we have

$$
\frac{\left|z_{\alpha_{n}^{k n} \beta_{n},-}-z_{\alpha_{n}^{k_{n}} \beta_{n},+}\right|}{\left|\lambda_{n}\right|^{2}-1} \geq \frac{\left|\zeta_{\alpha_{n}^{k_{n}} \beta_{n}}-1\right|}{\left|\lambda_{n}\right|^{2}-1}-\frac{\rho^{\prime}}{\left|\operatorname{tr}\left(\beta_{n}\right)\right|\left(\left|\lambda_{n}\right|^{2}-1\right)} .
$$


By the condition $\left(i_{1}\right)$ we have

$$
\frac{\left|\zeta_{\alpha_{n}^{k_{n}} \beta_{n}}-1\right|}{\left|\lambda_{n}\right|^{2}-1}=\frac{\left|\zeta_{\alpha_{n}^{k_{n}} \beta_{n}}-1\right|}{\left|\lambda_{n}\right|^{2}-\left|\zeta_{\alpha_{n}^{k_{n}} \beta_{n}}\right|+\left|\zeta_{\alpha_{n}^{k_{n}} \beta_{n}}\right|-1}>\frac{1}{M+1}, \quad \text { for some } M>0 \text {. }
$$

Hence for large $n$ there exists $\kappa>0$ such that

$$
\frac{\left|z_{\alpha_{n}^{k_{n}} \beta_{n},-}-z_{\alpha_{n}^{k_{n}} \beta_{n},+}\right|}{\left|\lambda_{n}\right|^{2}-1}>\frac{1}{M+1}-\frac{\rho^{\prime}}{\left|\operatorname{tr}\left(\beta_{n}\right)\right|\left(\left|\lambda_{n}\right|^{2}-1\right)}>\kappa .
$$

For the upper bounds we have

$$
\left|z_{\alpha_{n}^{k n} \beta_{n},-}-z_{\alpha_{n}^{k n} \beta_{n},+}\right|<2\left|\lambda_{n}\right|+\rho^{\prime \prime}\left|\operatorname{tr}\left(\beta_{n}\right)\right|^{-1} .
$$

Note that $\operatorname{dist}\left(\mathcal{L}_{\alpha_{n}}, \mathcal{L}_{\alpha_{n}^{k n} \beta_{n}}\right)=\inf \left\{\operatorname{dist}\left(h_{1}, h_{2}\right) \mid h_{1} \in \mathcal{L}_{\alpha_{n}}, h_{2} \in \mathcal{L}_{\alpha_{n}{ }_{n} \beta_{n}}\right\}$. Set $h_{j}=$ $\left(z_{j}, \theta_{j}\right), j=1,2$. Then for an upper bound we can take

$$
\begin{aligned}
& \left(z_{1}, \theta_{1}\right)=\left(0,\left|z_{\alpha_{n}^{k n} \beta_{n}, l}\right|+\frac{1}{2}\left|z_{\alpha_{n}^{k_{n}} \beta_{n}, u}-z_{\alpha_{n}^{k_{n}} \beta_{n}, l}\right|\right), \\
& \left(z_{2}, \theta_{2}\right)=\left(\frac{1}{2}\left(z_{\alpha_{n}^{k n} \beta_{n}, u}+z_{\alpha_{n}^{k_{n}} \beta_{n}, l}\right), \frac{1}{2}\left|z_{\alpha_{n}^{k n} \beta_{n}, u}-z_{\alpha_{n}^{k_{n}} \beta_{n}, l}\right|\right) .
\end{aligned}
$$

By Lemma 4.2, $1-\sigma_{1}\left|\operatorname{tr}\left(\beta_{n}\right)\right|^{-1}<\left|z_{\alpha_{n}^{k n} \beta_{n}, l}\right| \leq\left|z_{\alpha_{n}^{k n} \beta_{n}, u}\right| \leq\left|\lambda_{n}\right|^{2}+\sigma_{2}\left|\operatorname{tr}\left(\beta_{n}\right)\right|^{-1}$ and above estimates for $\left|z_{\alpha_{n}^{k n} \beta_{n}, u}-z_{\alpha_{n}^{k n} \beta_{n}, l}\right|$ we have

$$
\begin{aligned}
& \cosh \operatorname{dist}\left(\mathcal{L}_{\alpha_{n}}, \mathcal{L}_{\alpha_{n}^{k n} \beta_{n}}\right) \\
& \leq \frac{\frac{1}{4}\left|z_{\alpha_{n}^{k n} \beta_{n}, u}+z_{\alpha_{n}^{k n} \beta_{n}, l}\right|^{2}+\left|z_{\alpha_{n}^{k n} \beta_{n}, l}\right|^{2}}{\left|z_{\alpha_{n}^{k n} \beta_{n}, u}-z_{\alpha_{n}^{k_{n}} \beta_{n}, l}\right|\left(\left|z_{\alpha_{n}^{k n} \beta_{n}, l}\right|+\frac{1}{2}\left|z_{\alpha_{n}^{k n} \beta_{n}, u}-z_{\alpha_{n}^{k n} \beta_{n}, l}\right|\right)}+1 \\
& <\frac{\left|\lambda_{n}\right|^{2}+\rho^{\prime \prime \prime}\left|\operatorname{tr}\left(\beta_{n}\right)\right|^{-1}+\kappa\left(\left|\lambda_{n}\right|^{2}-1\right)+1}{\kappa\left(\left|\lambda_{n}\right|^{2}-1\right)}, \quad \rho^{\prime \prime \prime}>0 .
\end{aligned}
$$

This last inequality implies the Lemma.

\section{Lemma 6.3}

$$
\lim _{n} \frac{1}{\left|\operatorname{tr}\left(\alpha_{n}^{k_{n}} \beta_{n}\right)\right|\left(\left|\lambda_{n}\right|^{2}-1\right)}=0
$$

Proof It follows from Proposition 4.1 and Lemma 6.2 that there exists $\rho>0$ such that

$$
\left|\operatorname{tr}\left(\alpha_{n}^{k_{n}} \beta_{n}\right)\right| \geq \rho\left(\frac{\left(\left|\lambda_{n}\right|^{2 D_{n}}+3\right)\left(\left|\lambda_{n}\right|^{2}-1\right)^{2 D_{n}}}{\left|\lambda_{n}\right|^{2 D_{n}}-1}\right)^{1 /\left(2 D_{n}\right)},
$$


hence we have

$$
\lim \frac{1}{\left|\operatorname{tr}\left(\alpha_{n}^{k_{n}} \beta_{n}\right)\right|\left(\left|\lambda_{n}\right|^{2}-1\right)} \leq \lim \rho^{\prime}\left(\frac{\left|\lambda_{n}\right|^{2 D_{n}}-1}{\left(\left|\lambda_{n}\right|^{2 D_{n}}+3\right)\left(\left|\lambda_{n}\right|^{2}-1\right)^{4 D_{n}}}\right)^{1 /\left(2 D_{n}\right)}
$$

Since $\left|\lambda_{n}\right|^{2 D_{n}}-1<\left|\lambda_{n}\right|^{2}-1$ for large $n$ we have

$$
\left.\lim \frac{1}{\left|\operatorname{tr}\left(\alpha_{n}^{k_{n}} \beta_{n}\right)\right|\left(\left|\lambda_{n}\right|^{2}-1\right)} \leq \lim \rho^{\prime \prime}\left(\left|\lambda_{n}\right|^{2}-1\right)^{(} 1-4 D_{n}\right) /\left(2 D_{n}\right)=0 .
$$

For large $n$ by condition (i $i_{1}$, we have $\delta\left(\left|\lambda_{n}\right|^{2}-1\right)<\left|\lambda_{n}\right|^{2}-\left|\zeta_{\alpha_{n}^{k_{n}} \beta_{n}}\right|$. Then by Lemma 4.2 ,

$$
|| z_{\alpha_{n}^{k n} \beta_{n}, u}|-| \zeta_{\alpha_{n}^{k n} \beta_{n}}|| \leq\left|z_{\alpha_{n}^{k n} \beta_{n}, u}-\zeta_{\alpha_{n}^{k_{n}} \beta_{n}}\right|<\frac{\chi}{\left|\operatorname{tr}\left(\beta_{n}\right)\right|}
$$

for some $\chi>0$ we have

$$
\left|\lambda_{n}\right|^{2}-\left|z_{\alpha_{n}^{k n} \beta_{n}, u}\right|>\left|\lambda_{n}\right|^{2}-\left|\zeta_{\alpha_{n}^{k n} \beta_{n}}\right|-\frac{\chi}{\left|\operatorname{tr}\left(\beta_{n}\right)\right|}>\delta\left(\left|\lambda_{n}\right|^{2}-1\right)-\frac{\chi}{\left|\operatorname{tr}\left(\beta_{n}\right)\right|}
$$

Set $\epsilon_{n}=\left(\delta\left(\left|\lambda_{n}\right|^{2}-1\right)-\chi /\left|\operatorname{tr}\left(\beta_{n}\right)\right|\right)$. Define Mobius transformations by

$$
\psi_{n}(x)=\left(1+\frac{\epsilon_{n}}{2\left|z_{\alpha_{n}^{k n} \beta_{n}, u}\right|}\right) \lambda_{n}^{-1}(x)
$$

Then

$$
\begin{aligned}
\left|\lambda_{n}\right|-\left|z_{\psi_{n}} \alpha_{n}^{k_{n}} \beta_{n} \psi_{n}^{-1, u}\right| & =\left|\lambda_{n}\right|-\left(1+\frac{\epsilon_{n}}{2\left|z_{\alpha_{n}^{k_{n}} \beta_{n}, u}\right|}\right)\left|\lambda_{n}\right|^{-1}\left|z_{\alpha_{n}^{k_{n}} \beta_{n}, u}\right| \\
& =\left(\left|\lambda_{n}\right|^{2}-\left|z_{\alpha_{n}^{k_{n}} \beta_{n}, u}\right|-\frac{\epsilon_{n}}{2}\right)\left|\lambda_{n}\right|^{-1} \\
& >\left(\epsilon_{n}-\frac{\epsilon_{n}}{2}\right)\left|\lambda_{n}\right|^{-1}=\frac{\epsilon_{n}}{2}\left|\lambda_{n}\right|^{-1} .
\end{aligned}
$$

Also by Lemma 4.2 and $\eta_{\alpha_{n}^{k n} \beta_{n}}=1$ we have

$$
|| z_{\alpha_{n}^{k n} \beta_{n}, l}|-1|<\frac{\chi^{\prime}}{\left|\operatorname{tr}\left(\beta_{n}\right)\right|}
$$


This gives

$$
\begin{aligned}
\left|z_{\psi_{n} \alpha_{n}^{k_{n}} \beta_{n} \psi_{n}^{-1}, l}\right| & \left|\lambda_{n}\right|^{-1} \\
& =\left(1+\frac{\epsilon_{n}}{2\left|z_{\alpha_{n}^{k_{n}} \beta_{n}, u}\right|}\right)\left|z_{\alpha_{n}^{k_{n}} \beta_{n}, l}\right|\left|\lambda_{n}\right|^{-1}-\left|\lambda_{n}\right|^{-1} \\
& =\left|\lambda_{n}\right|^{-1}\left(\left|z_{\alpha_{n}^{k n} \beta_{n}, l}\right|-1+\frac{\epsilon_{n}\left|z_{\alpha_{n}^{k n} \beta_{n}, l}\right|}{2\left|z_{\alpha_{n}^{k n} \beta_{n}, u}\right|}\right) \\
& >\left|\lambda_{n}\right|^{-1}\left(\frac{\epsilon_{n}\left|z_{\alpha_{n}^{k n} \beta_{n}, l}\right|}{2\left|z_{\alpha_{n}^{k n} \beta_{n}, u}\right|}-\frac{\chi^{\prime}}{\left|\operatorname{tr}\left(\beta_{n}\right)\right|}\right) \\
& >\frac{1}{\left|\lambda_{n}\right|\left|\operatorname{tr}\left(\beta_{n}\right)\right|}\left(\frac{\left|\operatorname{tr}\left(\beta_{n}\right)\right| \epsilon_{n}\left|z_{\alpha_{n}^{k n} \beta_{n}, l}\right|}{2\left|z_{\alpha_{n}^{k n} \beta_{n}, u}\right|}-\chi^{\prime}\right) \\
& >\frac{1}{\left|\lambda_{n}\right|\left|\operatorname{tr}\left(\beta_{n}\right)\right|}\left(\frac{\delta\left|\operatorname{tr}\left(\beta_{n}\right)\right|\left(\left|\lambda_{n}\right|^{2}-1\right)\left|z_{\alpha_{n}^{k n} \beta_{n}, l}\right|-\chi\left|z_{\alpha_{n}^{k_{n}} \beta_{n}, l}\right|}{2\left|z_{\alpha_{n}^{k n} \beta_{n}, u}\right|}-\chi^{\prime}\right) .
\end{aligned}
$$

By Lemma 6.3 and the above inequality we have

$$
\begin{aligned}
& \left|z_{\psi_{n} \alpha_{n}^{k n} \beta_{n} \psi_{n}^{-1, l}}\right|-\left|\lambda_{n}\right|^{-1}>0, \\
& \left|\operatorname{tr}\left(\alpha_{n}^{k_{n}} \beta_{n}\right)\right|\left(\left|z_{\psi_{n} \alpha_{n}^{k_{n}} \beta_{n} \psi_{n}^{-1}, l}\right|-\left|\lambda_{n}\right|^{-1}\right) \rightarrow \infty, \\
& \left|\operatorname{tr}\left(\alpha_{n}^{k_{n}} \beta_{n}\right)\right|\left(\left|\lambda_{n}\right|-\left|z_{\psi_{n}} \alpha_{n}^{k_{n}} \beta_{n} \psi_{n}^{-1}, u\right|\right) \rightarrow \infty .
\end{aligned}
$$

Hence,

$$
\begin{gathered}
\left|\lambda_{n}\right|^{-1}<\left|z_{\psi_{n} \alpha_{n}^{k_{n}} \beta_{n} \psi_{n}^{-1}, l}\right| \leq\left|z_{\psi_{n} \alpha_{n}^{k_{n}} \beta_{n} \psi_{n}^{-1}, u}\right|<\left|\lambda_{n}\right|, \\
\lim _{n}\left\{\frac{1}{\left(\left|z_{\psi_{n} \alpha_{n}^{k_{n}} \beta_{n} \psi_{n}^{-1}, u}\right|-\left|\lambda_{n}\right|\right)\left|\operatorname{tr}\left(\alpha_{n}^{k_{n}} \beta_{n}\right)\right|}, \frac{1}{\left(\left|z_{\alpha_{n}^{k_{n}} \beta_{n}, l}\right|-\left|\lambda_{n}\right|^{-1}\right)\left|\operatorname{tr}\left(\alpha_{n}^{k_{n}} \beta_{n}\right)\right|}\right\}=0 .
\end{gathered}
$$

The generators $\left\langle\psi_{n} \alpha_{n} \psi_{n}^{-1}, \psi_{n} \alpha_{n}^{k_{n}} \beta_{n} \psi_{n}^{-1}\right\rangle$ satisfy the conditions of Lemma 5.2 for large $n$.

Consider $\left(\mathrm{i}_{2}\right)$.

There exists $\left|\rho_{\alpha_{n}^{k n-1} \beta_{n}}\right| \rightarrow 1$ such that $\zeta_{\alpha_{n}^{k_{n}-1} \beta_{n}} \rho_{\alpha^{k_{n}-1} \beta_{n}}=\eta_{\alpha_{n}^{k_{n}-1} \beta_{n}}=1$. If

$$
\limsup _{n}\left|\rho_{\alpha_{n}^{k n-1} \beta_{n}}-1\right|>0,
$$


then (with the same index notation for subsequence there exists a subsequence) such that

$$
\liminf _{n}\left|\zeta_{\alpha_{n}^{k n-1} \beta_{n}}-\eta_{\alpha_{n}^{k_{n}-1} \beta_{n}}\right|>0 .
$$

This implies by Lemma 4.2,

$$
\liminf _{n}\left|z_{\alpha_{n}^{k n-1} \beta_{n},+}-z_{\alpha_{n}^{k_{n}-1} \beta_{n},-}\right|>0 .
$$

Hence by Proposition 4.1, there exists $\rho>0$ such that for large $n$,

$$
\left|\operatorname{tr}\left(\alpha_{n}^{k_{n}-1} \beta_{n}\right)\right| \geq \rho\left(\frac{\left|\lambda_{n}\right|^{2 D_{n}}+3}{\left|\lambda_{n}\right|^{2 D_{n}}-1}\right)^{1 /\left(2 D_{n}\right)} .
$$

In particular, we have $\left|\operatorname{tr}\left(\alpha_{n}^{k_{n}-1} \beta_{n}\right)\right| \rightarrow \infty$. Note

$$
\lim _{n} \frac{\left|\rho_{\alpha_{n}^{k n-1} \beta_{n}}\right|-1}{\left|\lambda_{n}\right|^{2}-1}=0 .
$$

This can be seen as follows: since $\left|\lambda_{n}\right|^{2}-\left|\zeta_{\alpha_{n}^{k n} \beta_{n}}\right| \leq\left|\lambda_{n}\right|^{2}-1$ we have either

$$
\frac{\left|\lambda_{n}\right|^{2}-\left|\zeta_{\alpha_{n}^{k_{n}} \beta_{n}}\right|}{\left|\lambda_{n}\right|^{2}-1} \rightarrow 0 \quad \text { or } \quad 1 \geq \frac{\left|\lambda_{n}\right|^{2}-\left|\zeta_{\alpha_{n}^{k n} \beta_{n}}\right|}{\left|\lambda_{n}\right|^{2}-1}>\epsilon>0
$$

Assume that the latter inequality holds. This is equivalent to $\left(i_{1}\right)$ and we follow the same idea used in $\left(\mathrm{i}_{1}\right)$. Set Mobius transformations $\psi_{n}(x)=\lambda_{n}^{-1}\left(1-\epsilon_{n}\right)^{-1} x$, with $\epsilon_{n}=\epsilon\left(\left|\lambda_{n}\right|^{2}-1\right) /\left(2\left|\lambda_{n}\right|^{2}\right)$. Then,

$$
\begin{aligned}
\left|\lambda_{n}\right|-\left|\zeta_{\psi_{n} \alpha_{n}^{k_{n}} \beta_{n} \psi_{n}^{-1}}\right| & =\left|\lambda_{n}\right|-\left|\lambda_{n}^{-1}\left(1-\epsilon_{n}\right)^{-1} \zeta_{\alpha_{n}^{k_{n}} \beta_{n}}\right| \\
& =\left|\lambda_{n}\right|^{-1}\left(1-\epsilon_{n}\right)^{-1}\left(\left|\lambda_{n}\right|^{2}\left(1-\epsilon_{n}\right)-\left|\zeta_{\alpha_{n}^{k_{n}} \beta_{n}}\right|\right) .
\end{aligned}
$$

Since $\left|\lambda_{n}\right|^{2}-\left|\zeta_{\alpha_{n}^{k n} \beta_{n}}\right|>\epsilon\left(\left|\lambda_{n}\right|^{2}-1\right)$ we have

$$
\left|\lambda_{n}\right|-\left|\zeta_{\psi_{n} \alpha_{n}^{k n} \beta_{n} \psi_{n}^{-1}}\right|>\left|\lambda_{n}\right|^{-1}\left(1-\epsilon_{n}\right)^{-1}\left(\epsilon\left(\left|\lambda_{n}\right|^{2}-1\right)-\left|\lambda_{n}\right|^{2} \epsilon_{n}\right)=\frac{\epsilon\left(\left|\lambda_{n}\right|^{2}-1\right)}{2\left|\lambda_{n}\right|\left(1-\epsilon_{n}\right)} .
$$

Since $\epsilon_{n} \rightarrow 0$, it follows from last inequality that for large $n$ we have

$$
\frac{\left|\lambda_{n}\right|-\left|\zeta_{\psi_{n}} \alpha_{n}^{k_{n}} \beta_{n} \psi_{n}^{-1}\right|}{\left|\lambda_{n}\right|^{2}-1}>\epsilon^{\prime}>0
$$

And $\eta_{\psi_{n} \alpha_{n}^{k n} \beta_{n} \psi_{n}^{-1}}=\lambda_{n}^{-1}\left(1-\epsilon_{n}\right)^{-1}$ we have

$$
\frac{\left|\eta_{\psi_{n}} \alpha_{n}^{k_{n}} \beta_{n} \psi_{n}^{-1}\right|-\left|\lambda_{n}\right|^{-1}}{|\lambda|^{2}-1}=\frac{\epsilon}{2\left|\lambda_{n}\right|^{3}\left(1-\epsilon_{n}\right)}>\epsilon^{\prime \prime}>0 .
$$


Since $\left|\operatorname{tr}\left(\alpha_{n}^{k_{n}} \beta_{n}\right)\left(\left|\lambda_{n}\right|^{2}-1\right)\right| \rightarrow \infty$, it follows that for large $n$,

$$
\left|\lambda_{n}\right|^{-1}<\left|\eta_{\psi_{n} \alpha_{n}^{k_{n}} \beta_{n} \psi_{n}^{-1}}\right| \leq\left|\zeta_{\psi_{n} \alpha_{n}^{k_{n}} \beta_{n} \psi_{n}^{-1}}\right|<\left|\lambda_{n}\right|
$$

$\lim _{n}\left\{\frac{1}{\left|\operatorname{tr}\left(\alpha_{n}^{k_{n}} \beta_{n}\right)\right|\left(\mid \eta_{\left.\left.\psi_{n} \alpha^{k_{n}} \beta_{n} \psi_{n}^{-1}|-| \lambda_{n}\right|^{-1}\right)}\right.}, \frac{1}{\left|\operatorname{tr}\left(\alpha_{n}^{k_{n}} \beta_{n}\right)\right|\left(\left|\zeta_{\psi_{n} \alpha_{n}^{k_{n}} \beta_{n} \psi_{n}^{-1}}\right|-\left|\lambda_{n}\right|\right)}\right\}=0$.

Hence by Lemma 4.2, $\left\langle\psi_{n} \alpha_{n} \psi_{n}^{-1}, \psi_{n} \alpha_{n}^{k_{n}} \beta_{n} \psi_{n}^{-1}\right\rangle$ satisfies Lemma 5.2.

If the former holds then

$$
\begin{aligned}
\left|\lambda_{n}\right|^{2}\left(1-\left|\zeta_{\alpha_{n}^{k n} \beta_{n}} \lambda_{n}^{-2}\right|\right) & =\left|\lambda_{n}\right|^{2}\left(1-\left|\zeta_{\alpha_{n}^{k n-1} \beta_{n}}\right|\right) \\
& =\left|\lambda_{n}\right|^{2}\left(\left|\zeta_{\alpha_{n}^{k_{n}-1} \beta_{n}} \rho_{\alpha^{k_{n}-1} \beta_{n}}\right|-\left|\zeta_{\alpha_{n}^{k n-1} \beta_{n}}\right|\right) \\
& =\left|\lambda_{n}\right|^{2}\left|\zeta_{\alpha_{n}^{k_{n}-1} \beta_{n}}\right|\left(\left|\rho_{\alpha^{k_{n}-1} \beta_{n}}\right|-1\right)
\end{aligned}
$$

The last equation implies that

$$
\lim _{n} \frac{\left|\rho_{\alpha_{n}^{k n-1} \beta_{n}}\right|-1}{\left|\lambda_{n}\right|^{2}-1}=0
$$

and $\left|\operatorname{tr}\left(\alpha_{n}^{k_{n}-1} \beta_{n}\right)\left(\left|\lambda_{n}\right|^{2}-1\right)\right| \rightarrow \infty$. It follows that for large $n$,

$$
\left|\lambda_{n}\right|^{-1}<\left|\zeta_{\alpha_{n}^{k n-1} \beta_{n}}\right| \leq\left|\eta_{\alpha_{n}^{k n-1} \beta_{n}}\right|<\left|\lambda_{n}\right|
$$

$$
\lim _{n}\left\{\frac{1}{\left|\operatorname{tr}\left(\alpha_{n}^{k_{n}-1} \beta_{n}\right)\right|\left(\left|\eta_{\alpha^{k_{n}-1} \beta_{n}}\right|-\left|\lambda_{n}\right|\right)}, \frac{1}{\left|\operatorname{tr}\left(\alpha_{n}^{k_{n}-1} \beta_{n}\right)\right|\left(\left|\zeta_{\alpha_{n}^{k n-1} \beta_{n}}\right|-\left|\lambda_{n}\right|^{-1}\right)}\right\}=0 .
$$

Hence by Lemma 4.2, $\left\langle\alpha_{n}, \alpha_{n}^{k_{n}-1} \beta_{n}\right\rangle$ satisfies Lemma 5.2.

Consider the case where $\rho_{\alpha_{n}^{k n-1} \beta_{n}} \rightarrow 1$.

Let $\psi_{n}$ be the Mobius transformations given by

where

$$
\psi_{n}(x)=\frac{1}{\Delta_{n}} \frac{{ }_{\alpha_{n}^{k_{n}-1} \beta_{n},-} x-z_{\alpha_{n}^{k n-1} \beta_{n},-}{ }^{k_{\alpha_{n}}^{k_{n}-1} \beta_{n},+}}{x-z_{\alpha_{n}^{k n-1} \beta_{n},-}}, \quad x \in \mathbb{C} .
$$

$$
\Delta_{n}=\frac{1}{z_{\alpha_{n}^{k n-1} \beta_{n},-} z_{\alpha_{n}^{k n-1} \beta_{n},+}-z_{\alpha_{n}^{k n-1} \beta_{n},-}^{2}} .
$$

Let $\phi_{n}$ be the Mobius transformations defined by

$$
\phi_{n}(x)=\eta_{\psi_{n} \alpha_{n}^{k_{n}} \beta_{n} \psi_{n}^{-1}}^{-1} x
$$


Then $\zeta_{\phi_{n} \psi_{n} \alpha_{n}^{k_{n}} \beta_{n} \psi_{n}^{-1} \phi_{n}^{-1}}$ is given by

$$
\zeta_{\phi_{n} \psi_{n} \alpha_{n}^{k n} \beta_{n} \psi_{n}^{-1} \phi_{n}^{-1}}=\left(\frac{1-z_{\alpha_{n}^{k n-1}}^{\beta_{n},-}}{1-z_{\alpha_{n}^{k n-1} \beta_{n},+}}\right)\left(\frac{\alpha_{n}^{k_{n}} \beta_{n}\left(z_{\alpha_{n}^{k n-1} \beta_{n},-}\right)-z_{\alpha_{n}^{k n-1} \beta_{n},+}}{\alpha_{n}^{k_{n}} \beta_{n}\left(z_{\alpha_{n}^{k_{n}-1} \beta_{n},+}\right)-z_{\alpha_{n}^{k_{n-1}} \beta_{n},-}}\right)
$$

To see this we do a simple computation. Set

$$
\tilde{\alpha}_{n}=\phi_{n} \psi_{n} \alpha_{n}^{k_{n}-1} \beta_{n} \psi_{n}^{-1} \phi_{n}^{-1} \quad \text { and } \quad \tilde{\beta}_{n}=\phi_{n} \psi_{n} \alpha_{n}^{k_{n}} \beta_{n} \psi_{n}^{-1} \phi_{n}^{-1} \text {. }
$$

Write the matrix

$$
\tilde{\beta}_{n}=\left(\begin{array}{ll}
\tilde{a}_{n} & \tilde{b}_{n} \\
\tilde{c}_{n} & \tilde{d}_{n}
\end{array}\right)
$$

Note that by our choice of $\phi_{n}$, we have $\eta_{\tilde{\beta}_{n}}=1$, so $\tilde{c}_{n}=-\tilde{d}_{n}$ and $\zeta_{\tilde{\beta}_{n}}=\tilde{a}_{n} /-\tilde{d}_{n}$. By straightforward matrix multiplications we have

$$
\begin{aligned}
& \tilde{a}_{n}=-z_{\alpha_{n}^{k n-1} \beta_{n},-}^{2} \lambda_{n}^{k_{n}} a_{n}-z_{\alpha_{n}^{k_{n}-1} \beta_{n},-} \lambda_{n}^{k_{n}} b_{n} \\
& +z_{\alpha_{n}^{k_{n}-1} \beta_{n},-} z_{\alpha_{n}^{k_{n}-1} \beta_{n},+}\left(z_{\alpha_{n}^{k n-1} \beta_{n},-} \lambda_{n}^{-k_{n}} c_{n}+\lambda_{n}^{-k_{n}} d_{n}\right) \text {, } \\
& \tilde{a}_{n}=\frac{\left(\frac{-z_{\alpha_{n}^{k n-1} \beta_{n,-}}\left(\lambda_{n}^{k n} a_{n} z_{\alpha_{n}^{k n-1} \beta_{n,-}}+\lambda_{n}^{k n} b_{n}\right)}{z_{\alpha_{n}^{k n-1} \beta_{n,-}} \lambda_{n}^{-k_{n}} c_{n}+\lambda_{n}^{-k_{n}} d_{n}}+z_{\alpha_{n}^{k n-1} \beta_{n},-} z_{\alpha_{n}^{k n-1} \beta_{n},+}\right)}{\left(z_{\alpha_{n}^{k}{ }^{k n-1} \beta_{n},-} \lambda_{n}^{-k_{n}} c_{n}+\lambda_{n}^{-k_{n}} d_{n}\right)^{-1}} \\
& =\frac{-z_{\alpha_{n}^{k n-1} \beta_{n},-}\left(\alpha_{n}^{k_{n}} \beta_{n}\left(z_{\alpha_{n}^{k n-1} \beta_{n},-}\right)-z_{\alpha_{n}^{k n-1} \beta_{n},+}\right)}{\left(z_{\alpha_{n}^{k n-1} \beta_{n},-} \lambda_{n}^{-k_{n}} c_{n}+\lambda_{n}^{-k_{n}} d_{n}\right)^{-1}} \text {, } \\
& \tilde{d}_{n}=z_{\alpha_{n}^{k_{n}-1} \beta_{n},-}{ }^{z} \alpha_{n}^{k_{n-1}} \beta_{n},+\lambda_{n}^{k_{n}} a_{n}+z_{\alpha_{n}^{k_{n}-1} \beta_{n},-} \lambda_{n}^{k_{n}} b_{n} \\
& -z_{\alpha_{n}^{k_{n}-1} \beta_{n},-}\left(z_{\alpha_{n}^{k_{n}-1} \beta_{n},+} \lambda_{n}^{k_{n}} c_{n} \lambda_{n}^{-k_{n}} d_{n}\right),
\end{aligned}
$$

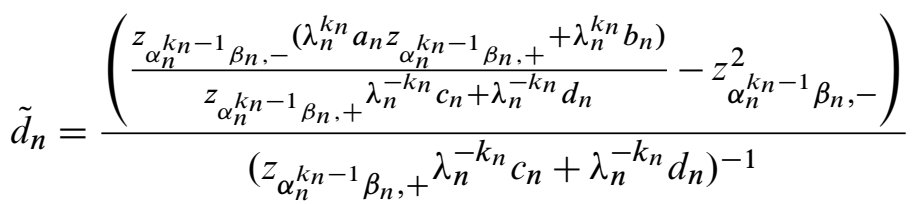

$$
\begin{aligned}
& =\frac{z_{\alpha_{n}^{k_{n-1}} \beta_{n,-}}\left(\alpha_{n}^{k_{n}} \beta_{n}\left(z_{\alpha_{n}^{k_{n-1}} \beta_{n},+}\right)-z_{\alpha_{n}^{k_{n-1}} \beta_{n},-}\right)}{\left(z_{\alpha_{n}^{k n-1} \beta_{n},+} \lambda_{n}^{-k_{n}} c_{n}+\lambda_{n}^{-k_{n}} d_{n}\right)^{-1}} \text {. }
\end{aligned}
$$


Now by $\eta_{n}=1$ we have

$$
\frac{z_{\alpha_{n}^{k n-1} \beta_{n},+} \lambda_{n}^{-k_{n}} c_{n}+\lambda_{n}^{-k_{n}} d_{n}}{z_{\alpha_{n}^{k n-1} \beta_{n},-} \lambda_{n}^{-k_{n}} c_{n}+\lambda_{n}^{-k_{n}} d_{n}}=\frac{1-z_{\alpha_{n}^{k n-1}} \beta_{n},+}{1-z_{\alpha_{n}^{k n-1}} \beta_{n},-} .
$$

Since $\zeta_{\tilde{\beta}_{n}}=\tilde{a}_{n} /-\tilde{d}_{n}$, the formula for $\zeta_{\tilde{\beta}_{n}}$ follows from the equations for $\tilde{a}_{n}$ and $\tilde{d}_{n}$. Let $\tilde{\lambda}_{n}$ denote the multiplier of $\tilde{\alpha}_{n}$.

First, we need to get a estimate of the growth of $\left|\operatorname{tr}\left(\tilde{\beta}_{n}\right)\right|$ in terms of $\left|\operatorname{tr}\left(\beta_{n}\right)\right|$. Note that $\left|\operatorname{tr}\left(\tilde{\beta}_{n}\right)\right|=\left|\operatorname{tr}\left(\alpha_{n}^{k_{n}} \beta_{n}\right)\right|$.

Remark 6.A There exists $\sigma, \sigma^{\prime}>0$ such that

$$
\sigma^{\prime}\left|\operatorname{tr}\left(\beta_{n}\right)\right|>\left|\operatorname{tr}\left(\tilde{\beta}_{n}\right)\right|>\sigma\left|\operatorname{tr}\left(\beta_{n}\right)\right|\left(\left|\lambda_{n}\right|^{2}-1\right) \quad \text { for } n \text { large. }
$$

In fact we only need the lower bound for $\left|\operatorname{tr}\left(\tilde{\beta}_{n}\right)\right|$.

Proof We have

$$
\frac{\left|\lambda_{n}\right|^{2}-1}{\left|\zeta_{\alpha_{n}^{k n} \beta_{n}}-1\right|}+\frac{\left|\zeta_{\alpha_{n}^{k n} \beta_{n}}\right|-\left|\lambda_{n}\right|^{2}}{\left|\zeta_{\alpha_{n}^{k_{n}} \beta_{n}}-1\right|}=\frac{\left|\zeta_{\alpha_{n}^{k_{n}} \beta_{n}}\right|-1}{\left|\zeta_{\alpha_{n}^{k_{n}} \beta_{n}}-1\right|} \leq 1
$$

and by condition $\left(i_{2}\right)$ we have

$$
\frac{\left|\zeta_{\alpha_{n}^{k n} \beta_{n}}\right|-\left|\lambda_{n}\right|^{2}}{\left|\zeta_{\alpha_{n}^{k n} \beta_{n}}-1\right|} \rightarrow 0
$$

thus

$$
\frac{\left|\zeta_{\alpha_{n}^{k n} \beta_{n}}-1\right|}{\left|\lambda_{n}\right|^{2}-1}>\epsilon>0, \quad \text { for large } n
$$

Recall $\eta_{\alpha_{n}^{k n} \beta_{n}}=1$, and by Lemma 4.7, more precisely by the second equation in the proof of Lemma 4.7 and $\left|\operatorname{tr}\left(\beta_{n}\right)\right| \asymp\left|c_{n}\right|$,

$$
\frac{\left|\operatorname{tr}\left(\alpha_{n}^{k_{n}} \beta_{n}\right)\right|}{\left|\operatorname{tr}\left(\beta_{n}\right)\right|}>\sigma^{\prime}\left|\zeta_{\alpha_{n}^{k_{n}} \beta_{n}}-\eta_{\alpha_{n}^{k_{n}} \beta_{n}}\right|>\sigma^{\prime} \epsilon\left(\left|\lambda_{n}\right|^{2}-1\right), \quad \text { for large } n .
$$

The upper bound is trivial.

Lemma 6.4 Assume that there exists a subsequence such that

$$
\lim _{j} \frac{\left|\zeta_{\tilde{\beta}_{n_{j}}}-1\right|}{\left|\tilde{\lambda}_{n_{j}}\right|^{2}-1}=0
$$

Then $\left\langle\tilde{\alpha}_{n_{j}}, \tilde{\beta}_{n_{j}}\right\rangle$ are classical Schottky generators for large $j$. 
Proof We will show that $\left\langle\tilde{\alpha}_{n_{j}}, \tilde{\beta}_{n_{j}}\right\rangle$ satisfies the conditions of Lemma 5.2 with Remark 5.4 .

Since by Remark 6.A, $\sigma^{\prime}\left|\operatorname{tr}\left(\beta_{n}\right)\right|>\left|\operatorname{tr}\left(\tilde{\beta}_{n}\right)\right|>\sigma\left|\operatorname{tr}\left(\beta_{n}\right)\right|\left(\left|\lambda_{n}\right|^{2}-1\right)$. In particular $\left|\operatorname{tr}\left(\tilde{\beta}_{n}\right)\right| \rightarrow \infty$, we have

$$
\lim _{j} \frac{\left|z_{\tilde{\beta}_{n_{j}},+}-z_{\tilde{\beta}_{n_{j}},-}\right|}{\left|\zeta_{\tilde{\beta}_{n_{j}}}-1\right|}=\lim _{j}\left|\frac{\sqrt{\left|\operatorname{tr}\left(\tilde{\beta}_{n_{j}}\right)\right|^{2}-4}}{\operatorname{tr}\left(\tilde{\beta}_{n_{j}}\right)}\right|=1 .
$$

Since $\left|\operatorname{tr}\left(\tilde{\beta}_{n_{j}}\right)\right| \rightarrow \infty$ the isometric circles of $\tilde{\beta}_{n_{j}}$ are disjoint for large $j$. Now

$$
\zeta_{\tilde{\beta}_{n_{j}}} \text { and } \eta_{\tilde{\beta}_{n_{j}}}
$$

are centers of these isometric circles and so by disjointness, the radius of these isometric circles must be less than

$$
\frac{\left|\zeta_{\tilde{\beta}_{n_{j}}}-\eta_{\tilde{\beta}_{n_{j}}}\right|}{2} \text { for large } j
$$

In addition, each isometric circle contains one of the fixed points

$$
z_{\tilde{\beta}_{n_{j}}, l} \text { or } z_{\tilde{\beta}_{n_{j}}, u} \text {. }
$$

By our convention $z_{\tilde{\beta}_{n_{j}}, l}, z_{\tilde{\beta}_{n_{j}}, u}$ are contained within the isometric circles with centers $\zeta_{\tilde{\beta}_{n_{j}}}, \eta_{\tilde{\beta}_{n_{j}}}$ respectively. Note that $\eta_{\tilde{\beta}_{n_{j}}}=1$. Hence for large $j$ we have

$$
\frac{\left|z_{\tilde{\beta}_{n_{j}}, l}-\zeta_{\tilde{\beta}_{n_{j}}}\right|}{\left|\zeta_{\tilde{\beta}_{n_{j}}}-1\right|}, \frac{\left|z_{\tilde{\beta}_{n_{j}}, u}-1\right|}{\left|\zeta_{\tilde{\beta}_{n_{j}}}-1\right|}<\frac{1}{2} .
$$

From these bounds we have

$$
\begin{aligned}
\lim _{j} \frac{\left|z_{\tilde{\beta}_{n_{j}}, l}-z_{\tilde{\beta}_{n_{j}}, u}\right|}{\left(\left|z_{\tilde{\beta}_{n_{j}}, u}\right|-\left|\tilde{\lambda}_{n_{j}}\right|\right)\left|\operatorname{tr}\left(\tilde{\beta}_{n_{j}}\right)\right|} & =\lim _{j} \frac{\left|\zeta_{\tilde{\beta}_{n_{j}}}-1\right|}{\left|\left(\left|z_{\tilde{\beta}_{n_{j}}, u}\right|-1\right)+\left(1-\left|\tilde{\lambda}_{n_{j}}\right|\right)\right|\left|\operatorname{tr}\left(\tilde{\beta}_{n_{j}}\right)\right|} \\
& \leq \lim _{j} \frac{1}{\left(\left|\frac{\tilde{\lambda}_{n_{j}} \mid-1}{\bar{\zeta}_{\tilde{\beta}_{n_{j}}}-1}\right|-\frac{1}{2}\right)\left|\operatorname{tr}\left(\tilde{\beta}_{n_{j}}\right)\right|}=0 .
\end{aligned}
$$


Similarly we have

$$
\begin{aligned}
& \lim _{j} \frac{\left|z_{\tilde{\beta}_{n_{j}}, l}-z_{\tilde{\beta}_{n_{j}}, u}\right|}{\left(\left|z_{\tilde{\beta}_{n_{j}}, l}\right|-\left|\tilde{\lambda}_{n_{j}}\right|^{-1}\right)\left|\operatorname{tr}\left(\tilde{\beta}_{n_{j}}\right)\right|} \\
& \quad=\lim _{j} \frac{\left|\zeta_{\tilde{\beta}_{n_{j}}}-1\right|}{\left|\left(\left|z_{\tilde{\beta}_{n_{j}}, u}\right|-\left|\zeta_{\tilde{\beta}_{n_{j}}}\right|\right)+\left(\left|\zeta_{\tilde{\beta}_{n_{j}}}\right|-1\right)+\left(1-\left|\tilde{\lambda}_{n_{j}}\right|-1\right)\right|\left|\operatorname{tr}\left(\tilde{\beta}_{n_{j}}\right)\right|} \\
& \quad \leq \lim _{j} \frac{1}{\left(\frac{1}{\left|\tilde{\lambda}_{n_{j}}\right|}\left|\frac{\tilde{\lambda}_{n_{j}} \mid-1}{\zeta_{\tilde{\beta}_{n_{j}}}-1}\right|+\left|\frac{\zeta_{\tilde{\beta}_{n_{j}}} \mid-1}{\zeta_{\tilde{\beta}_{n_{j}}}-1}\right|-\frac{1}{2}\right)\left|\operatorname{tr}\left(\tilde{\beta}_{n_{j}}\right)\right|}=0 .
\end{aligned}
$$

Hence by Remark 5.4, $\left\langle\tilde{\alpha}_{n_{j}}, \tilde{\beta}_{n_{j}}\right\rangle$ are classical Schottky generators for sufficiently large $j$.

Lemma 6.5 Assume that there exists $M>0$ such that

$$
M<\frac{\left|\zeta_{\tilde{\beta}_{n}}-1\right|}{\left|\tilde{\lambda}_{n}\right|^{2}-1}
$$

Then there exists $\mathcal{N}, \sigma>0$ such that

$$
\left|\tilde{\lambda}_{n}\right|^{2 D_{n}}-1>\sigma\left|\operatorname{tr}\left(\tilde{\beta}_{n}\right)\right|^{2 D_{n} /\left(2 D_{n}-1\right)} \quad \text { for } n>\mathcal{N} .
$$

Proof Use matrix representations we can write

$$
\tilde{\beta}_{n}=\left(\begin{array}{ll}
\tilde{a}_{n} & \tilde{b}_{n} \\
\tilde{c}_{n} & \tilde{d}_{n}
\end{array}\right) .
$$

Note that $\eta_{\tilde{\beta}_{n}}=1$. So $\tilde{c}_{n}=-\tilde{d}_{n}$ and we have

$$
\tilde{\beta}_{n}=\left(\begin{array}{cc}
\tilde{a}_{n} & \tilde{b}_{n} \\
-\tilde{d}_{n} & \tilde{d}_{n}
\end{array}\right)
$$

By $\left|\zeta_{\tilde{\beta}_{n}}\right| \leq 1$ and our assumption on $M$, we have

$$
M\left(\left|\tilde{\lambda}_{n}^{2}\right|-1\right)<\left|\zeta_{\tilde{\beta}_{n}}-1\right|<M^{\prime} .
$$

By $\left|\operatorname{tr}\left(\tilde{\beta}_{n}\right)\right| \rightarrow \infty$ and as in the proof of Lemma 6.4 we have

$$
\lim _{n} \frac{\left|z_{\tilde{\beta}_{n},+}-z_{\tilde{\beta}_{n},-}\right|}{\left|\zeta_{\tilde{\beta}_{n}}-1\right|}=\lim _{n}\left|\frac{\sqrt{\left|\operatorname{tr}\left(\tilde{\beta}_{n}\right)\right|^{2}-4}}{\operatorname{tr}\left(\tilde{\beta}_{n}\right)}\right|=1 .
$$


Since $\left|z_{\tilde{\beta}_{n},+}-z_{\tilde{\beta}_{n},-}\right|=\left|\tilde{d}_{n}\right|^{-1}\left|\sqrt{\operatorname{tr}^{2} \tilde{\beta}_{n}-4}\right|$ it follows that for some $\sigma, \sigma^{\prime}>0$ and large $n$,

$$
\sigma\left|\operatorname{tr}\left(\tilde{\beta}_{n}\right)\right|<\left|\tilde{d}_{n}\right|<\sigma^{\prime}\left|\operatorname{tr}\left(\tilde{\beta}_{n}\right)\right|\left(\left|\tilde{\lambda}_{n}\right|^{2}-1\right)^{-1}
$$

Let $e$ be the Euler number. If there exists a subsequence such that $\lim _{i}\left|\zeta_{\tilde{\beta}_{n_{i}}}\right|=e^{-1}$, then $1-e^{-1}<\left|z_{\tilde{\beta}_{n_{i}},+}-z_{\tilde{\beta}_{n_{i}},-}\right|<1+e^{-1}$ for large $n$. Otherwise we let $m_{n}>0$ be integers defined as

$$
1+\frac{1}{m_{n}+1} \leq\left|\tilde{\lambda}_{n}\right| \leq 1+\frac{1}{m_{n}} .
$$

From this definition we have $\lim _{n}\left|\tilde{\lambda}_{n}\right|^{m_{n}}=\lim _{n}\left(1+m_{n}^{-1}\right)^{m_{n}}=e$. Then there exists $N, \delta>0$ such that for $n>N$ we have

$$
\begin{aligned}
\left|\zeta_{\tilde{\alpha}_{n}^{m n} \tilde{\beta}_{n}}-\eta_{\tilde{\alpha}_{n}^{m n} \tilde{\beta}_{n}}\right| & \leq\left.|| \zeta_{\tilde{\beta}_{n}}|| \tilde{\lambda}_{n}\right|^{m_{n}}+1 \mid \\
& \leq \delta(e+1), \\
\left|\zeta_{\tilde{\alpha}_{n}^{m n} \tilde{\beta}_{n}}-\eta_{\tilde{\alpha}_{n}^{m n} \tilde{\beta}_{n}}\right| & \geq\left.|| \zeta_{\tilde{\beta}_{n}}|| \tilde{\lambda}_{n}\right|^{m_{n}}-1 \mid \\
& \geq \delta(e-1) .
\end{aligned}
$$

Hence it follows from Lemma 4.2 that there exists $\kappa>0$ such that

$$
\kappa^{-1}<\left|z_{\tilde{\alpha}_{n}^{m_{n}} \tilde{\beta}_{n},+}-z_{\tilde{\alpha}_{n}^{m_{n}} \tilde{\beta}_{n},-}\right|<\kappa .
$$

Therefore by setting $m_{n}=0$ for the subsequence with $\lim _{i}\left|\zeta_{\tilde{\beta}_{n_{i}}}\right|=e^{-1}$, we can always assume that for large $n$,

$$
\kappa^{-1}<\left|z_{\tilde{\alpha}_{n}^{m n}} \tilde{\beta}_{n},+-z_{\tilde{\alpha}_{n}^{m n} \tilde{\beta}_{n},-}\right|<\kappa .
$$

Since

$$
\left|z_{\tilde{\alpha}_{n}^{m} \tilde{\beta}_{n},+}-z_{\tilde{\alpha}_{n}^{m} \tilde{\beta}_{n},-}\right|=\frac{\left|\sqrt{\operatorname{tr}^{2}\left(\tilde{\alpha}_{n}^{m_{n}} \tilde{\beta}_{n}\right)-4}\right|}{\left|\tilde{d}_{n} \tilde{\lambda}_{n}^{m_{n}}\right|},
$$

we have

$$
\sigma^{\prime \prime}\left|\operatorname{tr}\left(\tilde{\beta}_{n}\right)\right|<\left|\operatorname{tr}\left(\tilde{\alpha}_{n}^{m_{n}} \tilde{\beta}_{n}\right)\right|<\frac{\sigma^{\prime \prime \prime}\left|\operatorname{tr}\left(\tilde{\beta}_{n}\right)\right|}{\left(\left|\tilde{\lambda}_{n}\right|^{2}-1\right)} .
$$

Since

$$
\kappa^{-1}<\mid z_{\tilde{\alpha}_{n}^{m_{n}} \tilde{\beta}_{n},+}-z_{\tilde{\alpha}_{n}^{m n}} \tilde{\beta}_{n,-\mid<\kappa},
$$

we have $\operatorname{dist}\left(\mathcal{L}_{\tilde{\alpha}_{n}}, \mathcal{L}_{\tilde{\alpha}_{n}^{m n}} \tilde{\beta}_{n}\right)<\delta$ for some $\delta>0$. By Remark 4.1.A applied to $\left\langle\tilde{\alpha}_{n}, \tilde{\alpha}_{n}^{m_{n}} \tilde{\beta}_{n}\right\rangle$ we have

$$
\left|\operatorname{tr}\left(\tilde{\alpha}_{n}^{m_{n}} \tilde{\beta}_{n}\right)\right|>\rho\left(\frac{\left|\tilde{\lambda}_{n}\right|^{2 D_{n}+3}}{\left|\tilde{\lambda}_{n}\right|^{2 D_{n}-1}}\right)^{1 /\left(2 D_{n}\right)}
$$


and as an upper bound for $\left|\operatorname{tr}\left(\tilde{\alpha}_{n}^{m_{n}} \tilde{\beta}_{n}\right)\right|$ we have

$$
\begin{aligned}
\left|\operatorname{tr}\left(\tilde{\beta}_{n}\right)\right| & >\rho \sigma^{\prime \prime \prime-1}\left(\left|\tilde{\lambda}_{n}\right|^{2}-1\right)\left(\frac{\left|\tilde{\lambda}_{n}\right|^{2 D_{n}}+3}{\left|\tilde{\lambda}_{n}\right|^{2 D_{n}}-1}\right)^{1 /\left(2 D_{n}\right)}>\rho^{\prime} \frac{\left|\tilde{\lambda}_{n}\right|^{2}-1}{\left(\left|\tilde{\lambda}_{n}\right|^{2 D_{n}}-1\right)^{1 /\left(2 D_{n}\right)}} \\
& >\rho^{\prime \prime}\left(\left|\tilde{\lambda}_{n}\right|^{2 D_{n}}-1\right)^{1-1 /\left(2 D_{n}\right)} .
\end{aligned}
$$

The last inequality implies that

$$
\left|\tilde{\lambda}_{n}\right|^{2}-1>\left|\tilde{\lambda}_{n}\right|^{2 D_{n}}-1>\rho^{\prime \prime \prime}\left|\operatorname{tr}\left(\tilde{\beta}_{n}\right)\right|^{2 D_{n} /\left(2 D_{n}-1\right)} .
$$

Proposition 6.6 Assume ( $\left.\mathrm{i}_{2}\right)$. Suppose that there exists a $M>0$ such that

$$
M<\frac{\left|\zeta_{\tilde{\beta}_{n}}-1\right|}{\left|\tilde{\lambda}_{n}\right|^{2}-1}
$$

Then $\left\langle\alpha_{n}, \alpha^{k_{n}-1} \beta_{n}\right\rangle$ are classical generators for large $n$.

To prove Proposition 6.6 when $\lim \sup _{n}\left|\operatorname{tr}\left(\alpha_{n}^{k_{n}-1} \beta_{n}\right)\right|<\infty$ we use disjoint nonisometric circles for $\alpha_{n}^{k_{n}-1} \beta_{n}$ based on the following.

Proposition 6.7 Given any loxodromic transformation $\gamma$ with fixed points $\neq 0, \infty$ and multiplier $\lambda_{\gamma}^{2}$, there exists disjoint circles $\mathcal{S}_{o, r}, \mathcal{S}_{o^{\prime}, r^{\prime}}$ of center $o$ radius $r$ and center $o^{\prime}$ radius $r^{\prime}$ respectively such that

$$
\gamma\left(\operatorname{interior}\left(\mathcal{S}_{o, r}\right)\right) \cap \operatorname{interior}\left(\mathcal{S}_{o^{\prime}, r^{\prime}}\right)=\varnothing \quad \text { and } \quad r+r^{\prime}=\left|z_{\gamma,+}-z_{\gamma,-}\right| \frac{2\left|\lambda_{\gamma}\right|}{\left|\lambda_{\gamma}\right|^{2}-1} .
$$

Note that since $\left|\lambda_{\gamma}\right|>1$, so by this equality for $r+r^{\prime}$ we have an upper bound

$$
r+r^{\prime}<\left|z_{\gamma,-}-z_{\gamma,+}\right| \frac{\left|\lambda_{\gamma}\right|+1}{\left|\lambda_{\gamma}\right|-1}
$$

Proof We conjugate $\gamma$ into Mobius transformation $\gamma^{\prime}$ with fixed points $\{0, \infty\}$. Consider circles $\mathcal{S}_{0,\left|\lambda_{\gamma}\right|^{-1}}, \mathcal{S}_{0,\left|\lambda_{\gamma}\right|}$. The Mobius transformation $\phi(x)=(x-1) /(x+1)$ maps the fixed points of $\gamma^{\prime}$ which are $\{0, \infty\}$ to fixed points $\{-1,1\}$ respectively. In addition it maps $\mathcal{S}_{0,\left|\lambda_{\gamma}\right|^{-1}}, \mathcal{S}_{0,\left|\lambda_{\gamma}\right|}$ to $S_{z_{1}, r_{1}}, S_{z_{1}^{\prime}, r_{1}^{\prime}}$ respectively. Here we can use basic 
formulas to determine $z_{1}, z_{1}^{\prime}, r_{1}, r_{1}^{\prime}$ (see page 91 of [6]). Explicitly by [6] we have

$$
\begin{aligned}
r_{1} & =\left|\frac{-\left|\lambda_{\gamma}\right|^{-2}-1}{-\left|\lambda_{\gamma}\right|^{-2}+1}-\frac{\left|\lambda_{\gamma}\right|^{-1}-1}{\left|\lambda_{\gamma}\right|^{-1}+1}\right| \\
& =\frac{2\left|\lambda_{\gamma}\right|}{\left|\lambda_{\gamma}\right|^{2}-1}, \\
r_{1}^{\prime} & =\left|\frac{-\left|\lambda_{\gamma}\right|^{2}-1}{-\left|\lambda_{\gamma}\right|^{2}+1}-\frac{\left|\lambda_{\gamma}\right|-1}{\left|\lambda_{\gamma}\right|+1}\right| \\
& =\frac{2\left|\lambda_{\gamma}\right|}{\left|\lambda_{\gamma}\right|^{2}-1} .
\end{aligned}
$$

This gives

$$
r_{1}+r_{1}^{\prime}=\frac{4\left|\lambda_{\gamma}\right|}{\left|\lambda_{\gamma}\right|^{2}-1} \text {. }
$$

The distance between the centers is

$$
\left|z_{1}-z_{1}^{\prime}\right|=\left|\frac{-\left|\lambda_{\gamma}\right|^{-2}-1}{-\left|\lambda_{\gamma}\right|^{-2}+1}-\frac{-\left|\lambda_{\gamma}\right|^{2}-1}{-\left|\lambda_{\gamma}\right|^{2}+1}\right|=2 \frac{\left|\lambda_{\gamma}\right|^{2}+1}{\left|\lambda_{\gamma}\right|^{2}-1} .
$$

The equality $\left(\left|\lambda_{\gamma}\right|^{2}+1\right)-2\left|\lambda_{\gamma}\right|=\left(\left|\lambda_{\gamma}\right|-1\right)^{2}>0$ implies $\mathcal{S}_{z_{1}, r_{1}}, \mathcal{S}_{z_{1}^{\prime}}, r_{1}^{\prime}$ are disjoint. By conjugating $\phi \gamma^{\prime} \phi^{-1}$ with

$$
\psi(x)=x+\frac{z_{\gamma,+}+z_{\gamma,-}}{z_{\gamma,+}-z_{\gamma,-}}
$$

we map the fixed points $\{-1,1\}$ to

$$
\left\{\frac{2 z_{\gamma,-}}{z_{\gamma,+}-z_{\gamma,-}}, \frac{2 z_{\gamma,+}}{z_{\gamma,+}-z_{\gamma,+}}\right\} \text {. }
$$

Because $\psi(x)$ is a translation (ie Euclidean isometry), the circles are mapped to $\mathcal{S}_{z_{2}, r_{2}}, \mathcal{S}_{z_{2}^{\prime}, r_{2}^{\prime}}$ with same radius and preserves the disjointness. Finally conjugating $\psi \phi \gamma \phi^{-1} \psi^{-1}$ by $\theta(x)=\left(z_{\gamma,+}-z_{\gamma,-}\right) / 2$ maps

$$
\left\{\frac{2 z_{\gamma,-}}{z_{\gamma,+}-z_{\gamma,-}}, \frac{2 z_{\gamma,+}}{z_{\gamma,+}-z_{\gamma,+}}\right\}
$$

to $\left\{z_{\gamma,-}, z_{\gamma,+}\right\}$, and maps circles to $\mathcal{S}_{z_{3}, r_{3}}, \mathcal{S}_{z_{3}^{\prime}, r_{3}^{\prime}}$. Note that

$$
r_{3}, r_{3}^{\prime}=\left|z_{\gamma,+}-z_{\gamma,-}\right| \frac{\left|\lambda_{\gamma}\right|}{\left|\lambda_{\gamma}\right|^{2}-1}
$$


and preserves the disjointness. Note that $\gamma=\theta \psi \phi \gamma^{\prime} \phi^{-1} \psi^{-1} \theta^{-1}$. Hence the sum of the radius of the resulting disjointed circles is

$$
r_{3}+r_{3}^{\prime}=\left|z_{\gamma,-}-z_{\gamma,+}\right| \frac{2\left|\lambda_{\gamma}\right|}{\left|\lambda_{\gamma}\right|^{2}-1} .
$$

Proof of Proposition 6.6 First assume that $\lim \sup _{n}\left|\operatorname{tr}\left(\alpha_{n}^{k_{n}-1} \beta_{n}\right)\right|<\infty$. Let $\mathcal{S}_{o_{n}, r_{n}}$, $\mathcal{S}_{o_{n}^{\prime}, r_{n}^{\prime}}$ be the disjoint circles for $\alpha_{n}^{k_{n}-1} \beta_{n}$ given by Proposition 6.7. We will show that

$$
\lim _{n} \frac{r_{n}+r_{n}^{\prime}}{\left|\lambda_{n}\right|^{2}-1}=0
$$

Note that

$$
\begin{aligned}
\lim _{n} \frac{r_{n}+r_{n}^{\prime}}{\left|\lambda_{n}\right|^{2}-1} & \leq \lim _{n}\left|z_{\alpha_{n}^{k_{n-1}} \beta_{n},+}-z_{\alpha_{n}^{k_{n}-1} \beta_{n},-}\right| \frac{2\left|\tilde{\lambda}_{n}\right|}{\left(\left|\tilde{\lambda}_{n}\right|^{2}-1\right)\left(\left|\lambda_{n}\right|^{2}-1\right)} \\
& \leq \lim _{n}\left|z_{\alpha_{n}^{k_{n-1}} \beta_{n},+}-z_{\alpha_{n}^{k_{n}-1} \beta_{n},-}\right| \frac{2 C}{\left(\left|\tilde{\lambda}_{n}\right|^{2}-1\right)\left(\left|\lambda_{n}\right|^{2}-1\right)},
\end{aligned}
$$

where the second inequality holds since $\left|\operatorname{tr}\left(\alpha_{n}^{k_{n}-1} \beta_{n}\right)\right|<C$ for some $C>0$.

Since $\lim \sup _{n}\left|\operatorname{tr}\left(\alpha^{k_{n}-1} \beta_{n}\right)\right|<\infty$ and $\lim \sup _{n}\left|\lambda_{n}\right|^{k_{n}-1}<\infty$, we have by Lemma 4.7 ,

$$
\left|z_{\alpha_{n}^{k_{n}-1} \beta_{n},+}-z_{\alpha_{n}^{k_{n}-1} \beta_{n},-}\right| \asymp \frac{1}{\left|\operatorname{tr}\left(\beta_{n}\right)\right|} .
$$

By Proposition 4.1,

$$
\left|\operatorname{tr}\left(\beta_{n}\right)\right|^{2 D_{n}}>\rho^{D_{n}} \frac{\left|\lambda_{n}\right|^{2 D_{n}}+3}{\left|\lambda_{n}\right|^{2 D_{n}}-1}>\frac{4 \rho^{D_{n}}}{\left|\lambda_{n}\right|^{2}-1} .
$$

The last inequality follows from $\left|\lambda_{n}\right|^{2 D_{n}}-1<\left|\lambda_{n}\right|^{2}-1$ for large $n$.

By our assumption that

$$
M<\frac{\left|\zeta_{\tilde{\beta}_{n}}-1\right|}{\left|\tilde{\lambda}_{n}\right|^{2}-1}
$$

and Lemma 6.5 we have

$$
\begin{aligned}
\lim _{n} \frac{r_{n}+r_{n}^{\prime}}{\left|\lambda_{n}\right|^{2}-1} & \leq \lim _{n}\left(4 \rho^{D_{n}}\right)^{-1} M^{\prime}\left|\operatorname{tr}\left(\beta_{n}\right)\right|^{-1}\left|\operatorname{tr}\left(\beta_{n}\right)\right|^{2 D_{n}}\left|\operatorname{tr}\left(\tilde{\beta}_{n}\right)\right|^{2 D_{n} /\left(1-2 D_{n}\right)} \\
& <\lim _{n}\left(4 \rho^{D_{n}}\right)^{-1} \sigma^{2 D_{n} /\left(1-2 D_{n}\right)} M^{\prime}\left|\operatorname{tr}\left(\beta_{n}\right)\right|^{\left(D_{n}\left(6-4 D_{n}\right)-1\right) /\left(1-2 D_{n}\right)}=0,
\end{aligned}
$$

where the second inequality holds since $\left|\operatorname{tr}\left(\tilde{\beta}_{n}\right)\right|<\sigma\left|\operatorname{tr}\left(\beta_{n}\right)\right|$. 
Now the circles $\mathcal{S}_{o_{n}, r_{n}}$ contains one of $z_{\alpha_{n}^{k_{n}-1} \beta_{n},-}, z_{\alpha^{k_{n}-1} \beta_{n},+}$ and $\mathcal{S}_{o_{n}^{\prime}, r_{n}^{\prime}}$ contains the other fixed point, and since $\eta_{\alpha_{n}^{k_{n}-1} \beta_{n}}=1$ and $\left|\zeta_{\alpha_{n}}^{k_{n-1} \beta_{\eta}}\right| \rightarrow 1$, it follows from Lemma 4.2, we must have $\mathcal{S}_{o_{n}, r_{n}}, \mathcal{S}_{o_{n}^{\prime}, r_{n}^{\prime}}$ contained in the region between $1 /\left|\lambda_{n}\right|$ and $\left|\lambda_{n}\right|$ for large $n$. Hence we have classical generators for large $n$.

Now if there is a subsequence such that $\left|\operatorname{tr}\left(\alpha_{n_{i}}^{k_{n_{i}}-1} \beta_{n_{i}}\right)\right| \rightarrow \infty$, then $\left\langle\alpha_{n_{i}}, \alpha^{k_{n_{i}}-1} \beta_{n_{i}}\right\rangle$ satisfies conditions of Lemma 5.2.

6.2.2 $\left(\mathbf{A}_{2}\right)$ To prove $\left(A_{2}\right)$ we can follow the steps given in the proof of $\left(A_{1}\right)$, and do the appropriate modifications. Some of the estimates will be simpler because $\left|\lambda_{n}\right|>\lambda$ and so estimates involving $\left(\left|\lambda_{n}\right|^{2}-1\right)^{-1}$ will hold trivially. However to avoid too much reproduction of the previous proof of $\left(\mathrm{A}_{1}\right)$, we give here a alternative short cut proof of $\left(\mathrm{A}_{2}\right)$ instead.

Proof of $\left(\mathbf{A}_{2}\right)$ Suppose that there is a subsequence (use same index for subsequence) such that $\left|\operatorname{tr}\left(\alpha_{n}^{k_{n}} \beta_{n}\right)\right| \rightarrow \infty$. Then by our assumption of $\left(\mathrm{A}_{2}\right),\left|\zeta_{\alpha_{n}^{k_{n}} \beta_{n}}\right| \rightarrow 1$ and $\left|\lambda_{n}\right|>\lambda>1$ we have for large $n$,

$$
\begin{gathered}
\lim _{n}\left\{\frac{1}{\left|\operatorname{tr}\left(\alpha_{n}^{k_{n}} \beta_{n}\right)\right|\left(\left|\eta_{\alpha^{k_{n}-1} \beta_{n}}\right|-\lambda^{-1}\right)}, \frac{1}{\left|\operatorname{tr}\left(\alpha_{n}^{k_{n}} \beta_{n}\right)\right|\left(\left|\zeta_{\alpha_{n}^{k n} \beta_{n}}\right|-\lambda_{n}\right)}\right\}=\left|\zeta_{\alpha_{n}^{k_{n}} \beta_{n}}\right|<\lambda \\
\ln _{n}
\end{gathered}
$$

By Lemma 4.2, $\left\langle\alpha_{n}, \alpha_{n}^{k_{n}} \beta_{n}\right\rangle$ satisfies the second set of conditions of Lemma 5.2, hence classical.

Otherwise we have $\left|\operatorname{tr}\left(\alpha_{n}^{k_{n}} \beta_{n}\right)\right|<C$ for some $C>0$. Since $\left|\zeta_{\alpha_{n} k_{n} \beta_{n}}\right| \rightarrow 1$ and $\eta_{\alpha_{n}^{k_{n}} \beta_{n}}=$ 1, by Lemma 4.2 we have $\left|z_{\alpha_{n}^{k n} \beta_{n}, \pm}\right| \rightarrow 1$. Now by Remark 4.1.A and $\left|\operatorname{tr}\left(\alpha_{n}^{k_{n}} \beta_{n}^{\alpha_{n}}\right)\right|<C$ we must have $\operatorname{dist}\left(\mathcal{L}_{\alpha_{n}}, \mathcal{L}_{\alpha_{n}^{k_{n}} \beta_{n}}\right) \rightarrow \infty$. This implies that $\left|z_{\alpha_{n}^{k_{n}} \beta_{n},+}-z_{\alpha_{n}^{k_{n}} \beta_{n},-}\right| \rightarrow 0$. More precisely we have:

Lemma 6.8 Suppose $\left|z_{\alpha_{n}^{k_{n}} \beta_{n},+}-z_{\alpha_{n}^{k_{n}} \beta_{n},-}\right| \rightarrow 0$ and $\left|z_{\alpha_{n}^{k_{n}} \beta_{n}, \pm}\right| \rightarrow 1$. Then there exists $\delta>0$ such that

$$
\operatorname{dist}\left(\mathcal{L}_{\alpha_{n}}, \mathcal{L}_{\alpha_{n}^{k n} \beta_{n}}\right)<\log \left(\frac{\delta}{\left|z_{\alpha_{n}^{k n} \beta_{n},+}-z_{\alpha_{n}^{k n} \beta_{n},-}\right|}\right) .
$$


Proof By using hyperbolic distance formula, and since $\left|z_{\alpha_{n}^{k_{n}} \beta_{n},+}-z_{\alpha_{n}^{k_{n}} \beta_{n},-}\right| \rightarrow 0$ and $\left|z_{\alpha_{n}^{k n} \beta_{n}, \pm}\right| \rightarrow 1$, we have for large $n$,

$$
\begin{aligned}
\cosh & \operatorname{dist}\left(\mathcal{L}_{\alpha_{n}}, \mathcal{L}_{\alpha_{n}^{k_{n}} \beta_{n}}\right) \\
& <\frac{\left|z_{\alpha_{n}^{k n} \beta_{n}, u}\right|^{2}+\frac{1}{4}\left(\left|z_{\alpha_{n}^{k_{n}} \beta_{n}, u}+z_{\alpha_{n}^{k_{n}} \beta_{n}, l}\right|\right)^{2}}{\frac{1}{2}\left(\left|z_{\alpha_{n}^{k_{n}} \beta_{n}, l}\right|+\frac{1}{2}\left|z_{\alpha_{n}^{k n} \beta_{n}, u}-z_{\alpha_{n}^{k_{n}} \beta_{n}, l}\right|\right)\left(\left|z_{\alpha_{n}^{k_{n}} \beta_{n}, u}-z_{\alpha_{n}^{k_{n}} \beta_{n}, l}\right|\right)}+1 \\
& <\frac{\rho}{\left|z_{\alpha_{n}^{k n} \beta_{n}, u}-z_{\alpha_{n}^{k n} \beta_{n}, l}\right|} \text { for some } \rho>0 .
\end{aligned}
$$

Let $\mathcal{S}_{o, r_{n}}, \mathcal{S}_{o^{\prime}, r_{n}^{\prime}}$ be the circles given by Proposition 6.7.

Proposition 6.9 If $\left|\operatorname{tr}\left(\alpha_{n}^{k_{n}} \beta_{n}\right)\right|<C$ for some $C>0$, then we must have $\left(r_{n}+r_{n}^{\prime}\right) \rightarrow 0$.

Proof First note that we have showed $\left|\operatorname{tr}\left(\alpha_{n}^{k_{n}} \beta_{n}\right)\right|<C$ implies

$$
\begin{gathered}
\left|z_{\alpha_{n}^{k n} \beta_{n},+}-z_{\alpha_{n}^{k_{n}} \beta_{n},-}\right| \rightarrow 0 \text { and }\left|z_{\alpha_{n}^{k_{n}} \beta_{n}, \pm}\right| \rightarrow 1 \\
\psi_{n}(x)=\frac{x-z_{\alpha_{n}^{k n} \beta_{n},+}}{x-z_{\alpha_{n}^{k n} \beta_{n},-}}
\end{gathered}
$$

Set

Let $\lambda_{\alpha_{n}^{k_{n}} \beta_{n}}$ be the multiplier of $\psi_{n} \alpha_{n}^{k_{n}} \beta_{n} \psi_{n}^{-1}$. We have

$$
\left|\lambda_{\alpha_{n}}\right|^{2}>\left(\frac{\left|\lambda_{\alpha_{n}^{k n} \beta_{n}}\right|^{2 D_{n}}+3}{\left|\lambda_{\alpha_{n}^{k n} \beta_{n}}\right|^{2 D_{n}}-1}\right)^{1 / D_{n}}\left(e^{-2 \operatorname{dist}\left(\mathcal{L}_{\psi_{n} \alpha_{n} \psi_{n}^{-1}}, \mathcal{L}_{\psi_{n} \alpha_{n}^{k n} \beta_{n} \psi_{n}^{-1}}\right)}\right) .
$$

by Remark 4.1.A applied to $\psi_{n}\left\langle\alpha_{n}, \alpha_{n}^{k_{n}} \beta_{n}\right\rangle \psi_{n}^{-1}$. Since $\left\{z_{\psi_{n} \alpha_{n} \psi^{-1},+}, z_{\psi_{n} \alpha_{n}} \psi_{n}^{-1,-}\right\}=$ $\left\{1, z_{\alpha_{n}^{k n} \beta_{n},+} / z_{\alpha_{n}^{k n} \beta_{n},-}\right\}$ we have

$$
\begin{aligned}
& \left|z_{\psi_{n} \alpha_{n} \psi_{n}^{-1, \pm}}\right| \rightarrow 1, \\
& \left|z_{\psi_{n} \alpha_{n} \psi_{n}^{-1},+}-z_{\psi_{n} \alpha_{n} \psi_{n}^{-1},-}\right|=\left|1-\frac{z_{\alpha_{n}^{k_{n}} \beta_{n},+}}{z_{\alpha_{n}^{k_{n}} \beta_{n},-}}\right| \rightarrow 0 .
\end{aligned}
$$

By Lemma 6.8 we have for large $n$,

$$
\operatorname{dist}\left(\mathcal{L}_{\psi_{n} \alpha_{n} \psi_{n}^{-1}}, \mathcal{L}_{\psi_{n} \alpha_{n}^{k_{n}} \beta_{n} \psi_{n}^{-1}}\right)<\log \left(\frac{\delta}{\left|z_{\psi_{n} \alpha_{n} \psi_{n}^{-1},+}-z_{\psi_{n} \alpha_{n} \psi_{n}^{-1},-}\right|}\right)
$$


This implies that for large $n$,

$$
\begin{aligned}
& \left|\lambda_{\alpha_{n}}\right|>\delta^{-1}\left(\frac{\left|\lambda_{\alpha_{n}^{k_{n}} \beta_{n}}\right|^{2 D_{n}}+3}{\left|\lambda_{\alpha_{n}^{k_{n}} \beta_{n}}\right|^{2 D_{n}-1}}\right)^{1 /\left(2 D_{n}\right)}\left|z_{\psi_{n} \alpha_{n} \psi_{n}^{-1},+}-z_{\psi_{n} \alpha_{n} \psi_{n}^{-1},-}\right| \\
& >\delta^{-1}\left(\left|\lambda_{\alpha_{n}^{k_{n}} \beta_{n}}\right|^{2 D_{n}}+3\right)^{1 /\left(2 D_{n}\right)}\left(\frac{\left|z_{\psi_{n} \alpha_{n} \psi_{n}^{-1},+}-z_{\psi_{n} \alpha_{n} \psi_{n}^{-1},-}\right|}{\left|\lambda_{\alpha_{n}^{k n} \beta_{n}}\right|^{2}-1}\right) \\
& >\delta^{-1}\left(\left|\lambda_{\alpha_{n}^{k_{n} \beta_{n}}}\right|^{2 D_{n}}+3\right)^{1 /\left(2 D_{n}\right)} \frac{\left(r_{n}+r_{n}^{\prime}\right)}{2 C^{\prime}\left|z_{\alpha_{n}^{k_{n}} \beta_{n},-}\right|}
\end{aligned}
$$

The last inequality follows from Proposition 6.7 and $\left|\lambda_{\alpha_{n}^{k n} \beta_{n}}\right|<C^{\prime}$. The second inequality in the above calculations follows since $\left|\lambda_{\alpha_{n}^{k n} \beta_{n}}\right|^{n}<C^{\prime}$ and for large $n$ we have

$$
\left(\left|\lambda_{\alpha_{n}^{k_{n} \beta_{n}}}\right|^{2 D_{n}}-1\right)^{1 /\left(2 D_{n}\right)} \leq\left|\lambda_{\alpha_{n}^{k_{n}} \beta_{n}}\right|^{2 D_{n}}-1 \leq\left|\lambda_{\alpha_{n}^{k_{n}} \beta_{n}}\right|^{2}-1 .
$$

Since $\left|\lambda_{\alpha_{n}}\right|<M$ for some $M$,

$$
r_{n}+r_{n}^{\prime}<\frac{2 C^{\prime} M \delta\left|z_{\alpha_{n}^{k_{n}} \beta_{n},-}\right|}{\left(\left|\lambda_{\alpha_{n}^{k_{n}} \beta_{n}}\right|^{2 D_{n}}+3\right)^{1 /\left(2 D_{n}\right)}}<\frac{2 C^{\prime} M \delta^{\prime}}{4^{1 /\left(2 D_{n}\right)}} \rightarrow 0 .
$$

Now we can continue and finish the proof for $\left|\operatorname{tr}\left(\alpha_{n}^{k_{n}} \beta_{n}\right)\right|<C$. By Proposition 6.9 and $\left|\lambda_{n}\right|>\lambda>1$ (this is the condition of $\left(\mathrm{A}_{2}\right)$ ) we have

$$
\frac{r_{n}+r_{n}^{\prime}}{\left|\lambda_{n}\right|^{2}-1} \rightarrow 0
$$

Since the circles $\mathcal{S}_{o_{n}, r_{n}}$ contains one of $z_{\alpha_{n}^{k n} \beta_{n},-}, z_{\alpha^{k n} \beta_{n},+}$ and $\mathcal{S}_{o_{n}^{\prime}, r_{n}^{\prime}}$ contains the other fixed point, and $\eta_{\alpha_{n}^{k_{n}} \beta_{n}}=1$ and $\left|\zeta_{\alpha_{n}}^{k_{n} \beta_{n}}\right| \rightarrow 1$ (condition of $\left(\mathrm{A}_{2}\right)$ ), it follows from Lemma 4.2, we must have $\mathcal{S}_{o_{n}, r_{n}}, \mathcal{S}_{o_{n}^{\prime}}, r_{n}^{\prime}$ contained in the region between $1 /\left|\lambda_{n}\right|$ and $\left|\lambda_{n}\right|$ for large $n$. Hence we have classical generators for large $n$. This completes the proof for $\left(\mathrm{A}_{2}\right)$.

\subsection{Case (B)}

Set Mobius transformations

$$
\psi_{n}(x)=\zeta_{n}^{-1} \lambda_{n}^{2-2 k_{n}} x
$$

and consider the generators $\psi_{n}\left\langle\alpha_{n}, \alpha^{k_{n}-1} \beta_{n}\right\rangle \psi_{n}^{-1}$. Then

$$
\zeta_{\psi_{n} \alpha_{n}^{k n-1} \beta_{n} \psi_{n}^{-1}}=1 \quad \text { and } \quad \eta_{\psi_{n} \alpha_{n}^{k_{n}} \beta_{n} \psi_{n}^{-1}}=\zeta_{n}^{-1} \lambda_{n}^{2-2 k_{n}}
$$


Since $1 \leq \zeta_{n} \lambda_{n}^{2 k_{n}}<\lambda_{n}^{2}$ and $\left|\zeta_{n} \lambda_{n}^{2 k_{n}}\right|-\left|\lambda_{n}^{2}\right| \rightarrow 0$ (the condition of (B)) we have

$$
\left|\zeta_{n} \lambda_{n}^{2 k_{n}}\right|\left(1-\left|\zeta_{n}^{-1} \lambda_{n}^{2-2 k_{n}}\right|\right) \rightarrow 0 \text {. }
$$

Since $\left|\lambda_{n}\right|<M$ for some $M>0$, we have $\left|\eta_{\psi_{n}} \alpha_{n}^{k_{n}-1} \beta_{n} \psi_{n}^{-1}\right| \rightarrow 1$. Hence by considering $\left\langle\psi_{n} \alpha_{n} \psi_{n}^{-1}, \psi_{n} \beta_{n}^{-1} \alpha_{n}^{1-k_{n}} \psi_{n}^{-1}\right\rangle$ we have

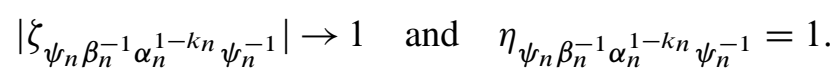

We have reduced $(\mathrm{B})$ to $(\mathrm{A})$.

Hence we have completed our proof of Theorem 6.1.

\section{$7 \Gamma$ with small $D_{\Gamma}$ is either classical or there exists a univer- sal lower bound on $Z_{\Gamma}$}

This section is devoted to proving Theorem 7.1 which will enable us to remove the constraint on $Z_{\Gamma_{n}}$ that was placed in the previous section.

Theorem 7.1 There exists $c>0$ such that if $\Gamma_{n}$ is a sequence of Schottky groups with $D_{n} \rightarrow 0$, Then for sufficiently large $n$, either there exists a subsequence $\Gamma_{n_{i}}$ that are classical Schottky groups, or there exists a subsequence $\Gamma_{n_{j}}$ with generating set $\left\langle\alpha_{n_{j}}, \beta_{n_{j}}\right\rangle$ such that $Z_{\left\langle\alpha_{n_{j}}, \beta_{n_{j}}\right\rangle}>c$.

Proof We prove by contradiction. Suppose there exists $\Gamma_{n}$ a sequence of Schottky groups such that for every generating set $\left\langle\alpha_{n}, \beta_{n}\right\rangle$ of $\Gamma_{n}$ we have $Z_{\left\langle\alpha_{n}, \beta_{n}\right\rangle} \rightarrow 0$.

For each $n$, by replacing $\left\langle\alpha_{n}, \beta_{n}\right\rangle$ with $\left\langle\alpha_{n}, \alpha_{n}^{m_{n}} \beta_{n}\right\rangle$ for sufficiently large $m_{n}$ if necessary, we can always assume that every $\Gamma_{n}$ is generated by generators with $\left|\operatorname{tr}\left(\alpha_{n}\right)\right| \leq\left|\operatorname{tr}\left(\beta_{n}\right)\right|$ and $\left|\operatorname{tr}\left(\beta_{n}\right)\right| \geq \log 3 / D_{n}$.

We take the upper space model $\mathbb{H}^{3}$. By conjugating with Mobius transformations, we can assume that $\alpha_{n}$ have fixed points $0, \infty$ with multiplier $\lambda_{n}$, and $\beta_{n}$ with $z_{\beta_{n}, u}=1$. Recall that, as before we denote the two fixed points of $\beta_{n}$ by $z_{\beta_{n}, l}, z_{\beta_{n}, u}$, with $\left|z_{\beta_{n}, l}\right| \leq\left|z_{\beta_{n}, u}\right|$. When we write $\beta_{n}$ in matrix form, we assume that $\left|a_{n}\right| \leq\left|d_{n}\right|$, otherwise we replace $\beta_{n}$ with $\beta_{n}^{-1}$.

By assumption, we have two cases: either (A) $z_{\beta_{n}, l} \rightarrow 1$ or (B) $z_{\beta_{n}, l} \rightarrow 0$. First we consider Case (A).

\subsection{Case (A)}

There are two possibilities, $\left(\mathrm{A}_{1}\right) \liminf _{n}\left|\lambda_{n}\right|=1$ or $\left(\mathrm{A}_{2}\right)$ there exists $\lambda>1$ such that $\left|\lambda_{n}\right|>\lambda$. 
7.1.1 ( $\left.\mathbf{A}_{2}\right)$ Since $\left|\operatorname{tr}\left(\beta_{n}\right)\right| \rightarrow \infty$ and $z_{\beta_{n}, l} \rightarrow z_{\beta_{n}, u}=1$ and $\left|\lambda_{n}\right|>\lambda>1$, we have $1 / \lambda<\left|z_{\beta_{n}, l}\right| \leq\left|z_{\beta_{n}, u}\right|<\lambda$ for large $n$. Hence $\left\langle\alpha_{n}, \beta_{n}\right\rangle$ satisfies Lemma 5.2 for large $n$.

7.1.2 ( $\left.\mathbf{A}_{1}\right)$ Taking a subsequence if necessary, we may assume that $\left|\lambda_{n}\right|$ is strictly decreasing to 1 . For large enough $n$, we choose a sequence of positive integers $m_{n}$ depends on $n$ such that $1+1 /\left(m_{n}+1\right) \leq\left|\lambda_{n}\right| \leq 1+1 / m_{n}$. Let us set $\zeta_{n}=a_{n} / c_{n}$, $\eta_{n}=-d_{n} / c_{n}$. Since $\left|\operatorname{tr}\left(\beta_{n}\right)\right| \rightarrow \infty$ and $\left|\sqrt{\operatorname{tr}^{2}\left(\beta_{n}\right)-4} /\left(2 c_{n}\right)\right|=\left|z_{\beta_{n},+}-z_{\beta_{n},-}\right| \rightarrow 0$ implies $\left|\operatorname{tr}\left(\beta_{n}\right)\right|<c_{n}$, it follows from Lemma 4.2 and Remark 4.5 that

$$
\left|z_{\alpha_{n}^{m_{n}} \beta_{n}, \pm}-\zeta_{n} \lambda_{n}^{2 m_{n}}\right| \leq \rho \frac{\left|\lambda_{n}^{m_{n}}\right|}{\left|\operatorname{tr}\left(\beta_{n}\right)\right|} \leq \rho \frac{e}{\left|\operatorname{tr}\left(\beta_{n}\right)\right|}
$$

for large $n$. Also by Lemma 4.2 and Remark 4.5, and the assumption that $z_{\beta_{n}, l} \rightarrow$ $z_{\beta_{n}, u}=1$, we have both $\zeta_{n}, \eta_{n} \rightarrow 1$. Hence

$$
\left|z_{\alpha_{n}^{m_{n}} \beta_{n}, \pm}-\lambda_{n}^{2 m_{n}}\right|+\left|z_{\alpha_{n}^{m_{n}} \beta_{n}, \mp}-1\right| \rightarrow 0 .
$$

Since by our choice of $m_{n}$, we have $\left|\lambda_{n}^{2 m_{n}}\right| \rightarrow e^{2}$. It follows that $\left|z_{\alpha_{n}^{m}}^{m_{n}} \beta_{n}, \pm\right| \rightarrow e^{2}$ and $\left|z_{\alpha_{n}^{m_{n}} \beta_{n}, \mp}\right| \rightarrow 1$. Therefore, there exists $c>0$ such that $Z_{\left\langle\alpha_{n}, \alpha_{n}^{m_{n}} \beta_{n}\right\rangle}>c$ for sufficiently large $n$.

\subsection{Case (B)}

Here we have either $\left(\mathrm{B}_{1}\right) \liminf _{n}\left|\lambda_{n}\right|<\Lambda$ for some $\Lambda>1$, or $\left(\mathrm{B}_{2}\right) \liminf _{n}\left|\lambda_{n}\right| \rightarrow \infty$. We also assume that $\left|\zeta_{n}\right| \leq\left|\eta_{n}\right|$ as before.

7.2.1 $\left(\mathbf{B}_{1}\right)$ We will show that there exists integers $k_{n}$ such that $Z_{\left\langle\alpha_{n}, \alpha_{n}^{k n} \beta_{n}\right\rangle}>c$ for some $c>0$.

Take subsequence if necessary, we may assume that $\left|\lambda_{n}\right| \leq \Lambda$ for large $n$. Choose positive integers $k_{n}$ to be the smallest such that $e^{2} \leq\left|\zeta_{n} \lambda_{n}^{2 k_{n}}\right|$. Since $\left|\lambda_{n}\right| \leq \Lambda$, we must have some $\sigma>0$ such that $e^{2}<\left|\zeta_{n} \lambda_{n}^{2 k_{n}}\right|<\sigma$. We claim that there exists $0<\epsilon<e^{2}$ and $n>N_{\epsilon}$ such that $e^{2}-\epsilon<\left|z_{\alpha_{n}^{k n} \beta_{n},+}\right|,\left|z_{\alpha_{n}^{k_{n}} \beta_{n},-}\right|<\sigma+\epsilon$. To see this, we use Remark 4.2.B.

To prove the claim, note that since $\left|z_{\beta_{n},-}-z_{\beta_{n},+}\right|<1+\epsilon$ for some $\epsilon>0$ and large $n$, and also $\left|\operatorname{tr}\left(\beta_{n}\right)\right| \rightarrow \infty$, we have $\left|c_{n}\right| \rightarrow \infty$. By Remark 4.2.B we have $\left|z_{\beta_{n}, u}-\eta_{n}\right| \rightarrow 0$, hence $\eta_{n} \rightarrow 1$.

First we show that $\left|z_{\alpha_{n}^{k n} \beta_{n},+}-z_{\alpha^{k n} \beta_{n},-}\right| \not \rightarrow \infty$. Assume otherwise. Let $\rho_{n}$ be the center of the circle having $z_{\alpha_{n}^{k n} \beta_{n},+}$ and $z_{\alpha_{n}^{k_{n}} \beta_{n},-}$ as antipodal points. Since $\left|\zeta_{\alpha_{n}^{k n} \beta_{n}}+\eta_{\alpha_{n}^{k n} \beta_{n}}\right|<\sigma+1+\epsilon^{\prime}$ for some $\epsilon^{\prime}>0$ and large $n$, and

$$
\rho_{n}=\frac{z_{\alpha_{n}^{k n} \beta_{n},+}+z_{\alpha^{k n} \beta_{n},-}}{2}
$$


and

$$
z_{\alpha_{n}^{k n} \beta_{n},+}+z_{\alpha^{k n} \beta_{n},-}=\zeta_{\alpha_{n}^{k n} \beta_{n}}+\eta_{\alpha_{n}^{k n} \beta_{n}}
$$

we have $\left|\rho_{n}-\sigma^{\prime}\right|<\kappa$ for some $\kappa>0$ and $\sigma^{\prime}=\sigma+1+\epsilon^{\prime}$. Note that since $\left|\rho_{n}-\sigma^{\prime}\right| \leq$ $\left|\rho_{n}\right|+\left|\sigma^{\prime}\right|<\left(\sigma+1+\epsilon^{\prime}\right) / 2+\sigma+1+\epsilon^{\prime}$ we can take $\kappa=\frac{3}{2}\left(\sigma+1+\epsilon^{\prime}\right)$. This implies that $\operatorname{dist}\left(\mathcal{L}_{\alpha_{n}}, \mathcal{L}_{\alpha_{n}^{k_{n}} \beta_{n}}\right)<\delta$ for some $\delta>0$. By Remark 4.1.A we have $\left|\operatorname{tr}\left(\alpha_{n}^{k_{n}} \beta_{n}\right)\right| \rightarrow \infty$, and

$$
\left|\zeta_{\alpha_{n}^{k n} \beta_{n}}-\eta_{\alpha_{n}^{k n} \beta_{n}}\right|=\frac{\left|\operatorname{tr}\left(\alpha_{n}^{k_{n}} \beta_{n}\right)\right|}{\left|c_{n} \lambda_{n}^{-k_{n}}\right|},
$$

we get $\left|\operatorname{tr}\left(\alpha_{n}^{k_{n}} \beta_{n}\right)\right| \asymp\left|c_{n} \lambda_{n}^{-k_{n}}\right|$. But this implies that $\left|z_{\alpha_{n}^{k_{n}} \beta_{n},+}-z_{\alpha^{k_{n}} \beta_{n},-}\right|<C$ for some $C>0$, hence a contradiction.

Note that if $\left|z_{\alpha^{k_{n}} \beta_{n},+}-z_{\alpha^{k_{n}} \beta_{n},-}\right| \rightarrow 0$ then

$$
\left|z_{\alpha^{k n} \beta_{n}, \pm}\right| \rightarrow \frac{1}{2}\left|\zeta_{\alpha_{n}^{k_{n}} \beta_{n}}+\eta_{\alpha_{n}^{k_{n}} \beta_{n}}\right|=\frac{1}{2}\left|\zeta_{\alpha_{n}^{k_{n}} \beta_{n}}+1\right| .
$$

Since $\frac{1}{2}\left(e^{2}-1\right)<\frac{1}{2}\left|\zeta_{\alpha_{n}^{k n} \beta_{n}}+1\right|<\frac{1}{2} \sigma^{\prime}$ for large $n$, this implies that $\frac{1}{2}\left(e^{2}-1\right)<$ $\left|z_{\alpha^{k n} \beta_{n}, \pm}\right|<\frac{1}{2} \sigma^{\prime}$ for large $n$.

Finally if $c<\left|z_{\alpha_{n}^{k_{n}} \beta_{n},+}-z_{\alpha^{k_{n}} \beta_{n},-}\right|<c^{\prime}$ for some $c, c^{\prime}>0$, then by Remark 4.1.A we have $\left|\operatorname{tr}\left(\alpha_{n}^{k_{n}} \beta_{n}\right)\right| \rightarrow \infty$ which implies $\left|c_{n} \lambda_{n}^{-k_{n}}\right| \rightarrow \infty$. Hence by Remark 4.2.B we have $\left\{z_{\alpha_{n}^{k n} \beta_{n},+}, z_{\alpha^{k n} \beta_{n},-}\right\} \rightarrow\left\{\zeta_{\alpha_{n}^{k_{n}} \beta_{n}}, \eta_{\alpha_{n}^{k_{n}} \beta_{n}}\right\}$ which implies the claim.

With the claim true, there are two possibilities: $\left(\mathrm{B}_{1}^{\prime}\right) \liminf \left|z_{\alpha_{n}^{k n} \beta_{n},+}-z_{\alpha_{n}^{k n} \beta_{n},-}\right| \rightarrow 0$, or $\left(\mathrm{B}_{1}^{\prime \prime}\right) \liminf \inf _{n}\left|z_{\alpha_{n}^{k_{n}} \beta_{n},+}-z_{\alpha_{n}^{k_{n}} \beta_{n},-}\right|>0$. For $\left(\mathrm{B}_{1}^{\prime \prime}\right)$, we have $Z_{\left\langle\alpha_{n}, \alpha_{n}^{k_{n}} \beta_{n}\right\rangle}>c$, for some $c>0$ and large $n$.

Suppose $\left(\mathrm{B}_{1}^{\prime}\right)$ holds. By passing to subsequence if necessary, we take

$$
\left|z_{\alpha_{n}^{k n} \beta_{n},+}-z_{\alpha_{n}^{k n} \beta_{n},-}\right| \rightarrow 0 .
$$

If $\left|\lambda_{n}\right| \rightarrow 1$ then we choose positive integers $m_{n}$ as defined in Case $\left(\mathrm{A}_{1}\right)$. Then

$$
e^{2} \leq \zeta_{n} \lambda_{n}^{2 k_{n}+2 m_{n}}<e^{4} \quad \text { and } \quad \kappa_{1}<\left|z_{\alpha_{n}^{k_{n}+m_{n}} \beta_{n},+}-z_{\alpha_{n}^{k_{n}+m_{n}} \beta_{n},-}\right|<\kappa_{2}
$$

for some $0<\kappa_{1}, \kappa_{2}$. By Remark 4.1.A we have $\left|\operatorname{tr}\left(\alpha_{n}^{k_{n}+m_{n}} \beta_{n}\right)\right| \rightarrow \infty$. Hence by Remark 4.2.B we have

$$
\left\{z_{\alpha_{n}^{k n+m_{n}} \beta_{n},+}, z_{\alpha_{n}^{k_{n}+m_{n}} \beta_{n},-}\right\} \rightarrow\left\{\zeta_{\alpha_{n}^{k_{n}+m_{n}} \beta_{n}}, \eta_{\alpha_{n}^{k_{n}+m_{n}} \beta_{n}}\right\} .
$$

This implies that $e^{2} \leq\left|z_{\alpha_{n}^{k_{n}+m_{n}} \beta_{n}, \pm}\right|<e^{4}$ and $\left|z_{\alpha_{n}^{k n+m_{n}} \beta_{n}, \mp}\right| \rightarrow 1$. Hence there exists $c>0$ such that $Z_{\left\langle\alpha_{n}, \alpha_{n}^{k_{n}+m_{n}} \beta_{n}\right\rangle}>c$ for large $n$. 
If $\left|\lambda_{n}\right|>c>0$ then we take $m_{n}=1$. Then

$$
\delta_{1}<\left|z_{\alpha_{n}^{k_{n}+1} \beta_{n},+}-z_{\alpha_{n}^{k_{n}+1} \beta_{n},-}\right|<\delta_{2}
$$

for some $0<\delta_{1}, \delta_{2}$. And it follows from Remark 4.1.A and Remark 4.2.B we have that $Z_{\left\langle\alpha_{n}, \alpha_{n}^{k_{n}+1} \beta_{n}\right\rangle}>c$ for large $n$.

7.2.2 $\left(\mathbf{B}_{2}\right)$ By taking a subsequence of $\alpha_{n}$, we may assume that $\left|\lambda_{n}\right|$ is strictly increasing. Choose a sequence of largest integers $k_{n} \geq 0$ such that $\left|\zeta_{n} \lambda_{n}^{2 k_{n}}\right| \leq 1$. If $\lim \sup \left|\zeta_{n} \lambda_{n}^{2 k_{n}}\right|=1$ but $\lim \sup \left|\zeta_{n} \lambda_{n}^{2 k_{n}}-1\right| \neq 0$, then there is a subsequence $\left\langle\alpha_{n_{j}}, \alpha_{n_{j}}^{k_{n_{j}}} \beta_{n_{j}}\right\rangle$ of $\left\langle\alpha_{n}, \alpha_{n}^{k_{n}} \beta_{n}\right\rangle$ such that $\left.\liminf _{j} Z_{\left\langle\alpha_{n_{j}}, \alpha_{n_{j}} k_{n_{j}}\right.} \beta_{n_{j}}\right\rangle>0$.

If $\lim \sup \zeta_{n} \lambda_{n}^{2 k_{n}}=1$, then let $\left\langle\alpha_{n_{i}}, \alpha_{n_{i}}^{k_{n_{i}}} \beta_{n_{i}}\right\rangle$ be the subsequence of $\left\langle\alpha_{n}, \alpha_{n}^{k_{n}} \beta_{n}\right\rangle$ with $\lim _{i} \zeta_{n_{i}} \lambda_{n_{i}}^{2 k_{n_{i}}}=1$.

If lim sup $\left|\operatorname{tr}\left(\alpha_{n_{i}}^{k_{n_{i}}} \beta_{n_{i}}\right)\right|=\infty$, then by passing to a subsequence if necessary, for large $i$, $\alpha_{n_{i}}^{k_{n_{i}} \beta_{n_{i}}}$ will have disjoint isometric circles. Note $\left|\operatorname{tr}\left(\beta_{n_{i}}\right)\right| \rightarrow \infty, z_{\beta_{n_{i}}, u}=1$ and $z_{\beta_{n_{i}}, l} \rightarrow 0$, so $\left|z_{\beta_{n_{i}},+}-z_{\beta_{n_{i}},-}\right| \leq 1+\epsilon_{n}$ for $\epsilon_{n} \rightarrow 0$ (ie $\left|z_{\beta_{n_{i}},+}-z_{\beta_{n_{i}},-}\right|<c$, for $c>0$ ), we have $\left|c_{n_{i}}\right| \rightarrow \infty$. By Remark 4.2.B, $\lim _{i} \min \left\{\left|\eta_{n_{i}}-z_{\beta_{n_{i}},-}\right|,\left|\eta_{n_{i}}-z_{\beta_{n_{i}},+}\right|\right\} \rightarrow 0$. Since $\zeta_{\alpha_{n_{i}}^{k n} \beta_{n_{i}}} \rightarrow 1=\eta_{\alpha_{n_{i}}^{k n_{i}} \beta_{n_{i}}}$ and $\left|\operatorname{tr}\left(\alpha_{n_{i}}^{k_{n_{i}}} \beta_{n_{i}}\right)\right| \rightarrow \infty$ we have

$$
\left|\zeta_{\alpha_{n_{i}}^{k n_{i} \beta_{n_{i}}}}-\eta_{\alpha_{n_{i}}^{k n_{i} \beta_{n_{i}}}}\right|=\frac{\left|\operatorname{tr}\left(\alpha_{n_{i}}^{k_{n_{i}}} \beta_{n_{i}}\right)\right|}{\left|\lambda_{n_{i}}^{-k_{n_{i}}} c_{n_{i}}\right|} \rightarrow 0 \quad \text { and } \quad\left|\lambda_{n_{i}}^{-k_{n_{i}}} c_{n_{i}}\right| \rightarrow \infty
$$

Also

$$
\left|z_{\alpha_{n_{i}}^{k n_{i}} \beta_{n_{i}},+}-z_{\alpha_{n_{i}}^{k n_{i}} \beta_{n_{i}},-}\right|=\frac{\left|\sqrt{\operatorname{tr}^{2}\left(\alpha_{n_{i}}^{k_{n_{i}}} \beta_{n_{i}}\right)-4}\right|}{2\left|\lambda_{n_{i}}^{-k_{n_{i}}} c_{n_{i}}\right|} \rightarrow 0 .
$$

Hence by Remark 4.2.B, there exists a $\kappa>0$ such that for large $i$ we have

$$
\kappa^{-1}<\left|z_{\alpha_{n_{i}}^{k n_{i}} \beta_{n_{i}}, l}\right| \leq\left|z_{\alpha_{n_{i}}^{k n_{i}} \beta_{n_{i}}, u}\right|<\kappa .
$$

And since $\left|\lambda_{n_{i}}\right| \rightarrow \infty$, we are able to choose Mobius transformations $\psi_{i}$ such that

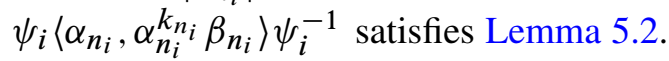

If $\lim \sup \left|\operatorname{tr}\left(\alpha_{n_{i}}^{k_{n_{i}}} \beta_{n_{i}}\right)\right|<\infty$, then let $\phi_{i}$ be Mobius transformations such that the maps $\phi_{i} \alpha_{n_{i}}^{k_{n_{i}}} \beta_{n_{i}} \phi_{i}^{-1}$ have fixed points $0, \infty$, and the fixed point $z_{\phi_{i} \alpha_{n_{i}} \phi_{i}^{-1}, u}$ of $\alpha_{n_{i}}$ is 1 . It follows that $z_{\phi_{i} \alpha_{n_{i}} \phi_{i}^{-1}, l} \rightarrow 1$. Since $\left|\operatorname{tr}\left(\phi_{i} \alpha_{n_{i}} \phi_{i}^{-1}\right)\right| \rightarrow \infty$, hence we have reduced this case to Case (A), which we already considered.

If $\lim \sup \left|\zeta_{n} \lambda_{n}^{2 k_{n}}\right|<1$, then we have two possibilities: (B' $\left.{ }_{2}^{\prime}\right) \lim \inf \left|\zeta_{n} \lambda_{n}^{2 k_{n}+2}\right|=1$, or $\left(\mathrm{B}_{2}^{\prime \prime}\right) \liminf \left|\zeta_{n} \lambda_{n}^{2 k_{n}+2}\right|>1$. 
Consider Case $\left(\mathrm{B}_{2}^{\prime}\right)$. Let $\left\langle\alpha_{n_{i}}, \alpha_{n_{i}}^{k_{n_{i}}} \beta_{n_{i}}\right\rangle$ be a subsequence of $\left\langle\alpha_{n}, \alpha_{n}^{k_{n}} \beta_{n}\right\rangle$ such that $\lim _{i}\left|\zeta_{n_{i}} \lambda_{n_{i}}^{2 k_{n_{i}}+2}\right| \rightarrow 1$. If $\sup _{i}\left|\operatorname{tr}\left(\alpha_{n_{i}}^{k_{n_{i}+1}} \beta_{n_{i}}\right)\right|<\infty$, then we conjugate $\alpha_{n_{i}}, \alpha_{n_{i}}^{k_{n_{i}}} \beta_{n_{i}}$ to $\hat{\beta}_{i}=\phi_{i} \alpha_{n_{i}} \phi_{i}^{-1}, \hat{\alpha}_{i}=\phi_{i} \alpha_{n_{i}}^{k_{n_{i}}+1} \beta_{n_{i}} \phi_{i}^{-1}$ with $\hat{\alpha}_{i}$ have fixed points $0, \infty$ and $\hat{\beta}_{i}$ have $z_{\hat{\beta}_{i}, u}=1$. Since $\sup _{i}\left|\hat{\lambda}_{i}\right|<\infty$, it follows that, if $z_{\hat{\beta}_{i}, l} \rightarrow 1$ then $\left\langle\hat{\alpha}_{i}, \hat{\beta}_{i}\right\rangle$, falls under Case (A), and if $z_{\hat{\beta}_{i}, l} \rightarrow 0$ then $\left\langle\hat{\alpha}_{i}, \hat{\beta}_{i}\right\rangle$ falls under Case $\left(\mathrm{B}_{1}\right)$. Otherwise there exists $\epsilon>0$ such that $\epsilon \stackrel{\hat{\beta}_{i}, l}{<} \hat{\beta}_{i}, l \mid<1-\epsilon$, hence $Z_{\left\langle\hat{\alpha}_{i}, \hat{\beta}_{i}\right\rangle}>c$ for some $c>0$.

On the other hand, if $\sup _{i}\left|\operatorname{tr}\left(\alpha_{n_{i}}^{k_{n_{i}}+1} \beta_{n_{i}}\right)\right|=\infty$, then for large $i$ since the radius of isometric circles is

$$
\Re_{\alpha_{n_{i}}^{k n_{i}+1} \beta_{n_{i}}}=\frac{\left|z_{\alpha_{n_{i}}^{k_{n_{i}}+1} \beta_{n_{i}}, u}-z_{\alpha_{n_{i}} k_{n_{i}+1}} \beta_{n_{i}, l}\right|}{\left|\operatorname{tr}\left(\alpha_{n_{i}}^{k_{n_{i}}+1} \beta_{n_{i}}\right)\right|}
$$

and the distance between the centers of these isometric circles is

$$
\begin{aligned}
& \left|\zeta_{\alpha_{n_{i}}^{k n_{i}+1} \beta_{n_{i}}}-\eta_{\alpha_{n_{i}}^{k n_{i}+1} \beta_{n_{i}}}\right|=\frac{\left|\operatorname{tr}\left(\alpha_{n_{i}}^{k_{n_{i}}+1} \beta_{n_{i}}\right)\right|}{c_{n} \lambda_{n_{i}}^{-k_{n_{i}}-1}}, \\
& \left|z_{\alpha_{n_{i}}^{k_{n_{i}+1}+1} \beta_{n_{i}}, u}-z_{\alpha_{n_{i}}^{k n_{n_{i}}+1} \beta_{n_{i}}, l}\right|=\frac{\sqrt{\operatorname{tr}^{2}\left(\alpha_{n_{i}}^{k_{n_{i}}+1} \beta_{n_{i}}\right)-4}}{2 c_{n_{i}} \lambda_{n_{i}}^{-k_{n_{i}}-1}}
\end{aligned}
$$

we have

$$
\lim _{i} \frac{\left|\zeta{ }_{\alpha_{n_{i}} k_{n_{i}+1} \beta_{n_{i}}}-\eta_{\alpha_{n_{i}} k_{n_{i}+1}} \beta_{n_{i}}\right|}{\Re_{\alpha_{n_{i}} k_{n_{i}+1}} \beta_{n_{i}}}=\lim _{i} \frac{2\left|\operatorname{tr}\left(\alpha_{n_{i}}^{k_{n_{i}}+1} \beta_{n_{i}}\right)\right|^{2}}{\sqrt{\operatorname{tr}^{2}\left(\alpha_{n_{i}}^{k_{n_{i}}+1} \beta_{n_{i}}\right)-4}}>\delta\left|\operatorname{tr}\left(\alpha_{n_{i}}^{k_{n_{i}}+1} \beta_{n_{i}}\right)\right|
$$

for some $\delta>0$. Hence

$$
\left|\zeta_{\alpha_{n_{i}}^{k n_{i}+1} \beta_{n_{i}}}-\eta_{\alpha_{n_{i}}^{k n_{i}+1} \beta_{n_{i}}}^{k}\right|>2 \mathfrak{R}_{\alpha_{n_{i}}^{k n_{i}+1}}^{k \beta_{n_{i}}}
$$

for large $i$. This implies $\alpha_{n_{i}}^{k_{n_{i}}+1} \beta_{n_{i}}$ have disjointed isometric circles for large $i$. By Lemma 4.2 and $z_{\beta_{n_{i}}, u}=1$ we have $\eta_{\beta_{n_{i}}} \rightarrow 1$. And since

$$
\eta_{\alpha_{n_{i}}^{k n_{i}+1}{ }_{\beta_{n_{i}}}}=\eta_{\beta_{n_{i}}}
$$

we have that

Note that if

$$
\eta_{\alpha_{n_{i}}^{k n_{i}+1} \beta_{n_{i}}} \rightarrow 1
$$

$$
\inf _{i}\left|\zeta_{\alpha_{n_{i}}^{k n_{i}+1} \beta_{n_{i}}}-1\right|>0 \text { and }\left|\zeta_{\alpha_{n_{i}} k_{n_{i}}+1}^{k \beta_{n_{i}}}\right| \rightarrow 1
$$


then by $\left|\operatorname{tr}\left(\alpha_{n_{i}}^{k_{n_{i}}+1} \beta_{n_{i}}\right)\right| \rightarrow \infty$ we have

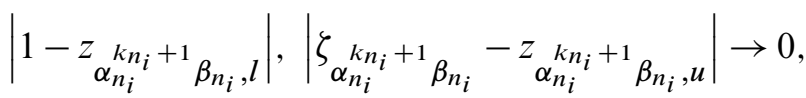

$$
\begin{aligned}
& \inf _{i}\left|z_{\alpha_{n_{i}}^{k n_{i}+1} \beta_{n_{i}}, u}^{k n_{n_{n_{i}}}^{k} n_{n_{i}+1} \beta_{n_{i}, l}, l}\right|>0 .
\end{aligned}
$$

Hence for large $i$ there exits $\epsilon>0$ such that

$$
1-\epsilon<\left|z_{\alpha_{n_{i}}^{k n_{i}+1} \beta_{n_{i}, l}, l}\right| \leq\left|z_{\alpha_{n_{i}}^{k n_{i}+1} \beta_{n_{i}}, u}\right|<1+\epsilon,
$$

therefore we must have

$$
\inf _{i} Z_{\left\langle\alpha_{n_{i}}, \alpha_{n_{i}}^{k n_{i}+1} \beta_{n_{i}}\right\rangle}>0
$$

It follows we can assume that

$$
\zeta_{\alpha_{n_{i}}^{k n_{i}}+\beta_{n_{i}}}^{k} \rightarrow 1
$$

Then we have

Hence

$$
\left|\zeta_{\alpha_{n_{i}}^{k n_{i}+1} \beta_{n_{i}}}^{k_{n}}-\eta_{\alpha_{n_{i}} k_{n_{i}}+1} \beta_{n_{i}}\right| \rightarrow 0
$$

$$
\frac{\left|\operatorname{tr}\left(\alpha_{n_{i}}^{k_{n_{i}}+1} \beta_{n_{i}}\right)\right|}{c_{n} \lambda_{n_{i}}^{-k_{n_{i}}-1}} \rightarrow 0
$$

Since $\left|\operatorname{tr}\left(\alpha_{n_{i}}^{k_{n_{i}}+1} \beta_{n_{i}}\right)\right| \rightarrow \infty$

$$
\left|z_{\alpha_{n_{i}}^{k_{n_{i}+1}} \beta_{n_{i}}, u}-z_{\alpha_{n_{i}}^{k_{n_{i}+1}+1} \beta_{n_{i}}, l}\right|=\frac{\sqrt{\operatorname{tr}^{2}\left(\alpha_{n_{i}}^{k_{n_{i}}+1} \beta_{n_{i}}\right)-4}}{2 c_{n_{i}} \lambda_{n_{i}}^{-k_{n_{i}}-1}} \rightarrow 0 .
$$

Therefore the distance between the centers of these isometric circles decreases to 0 and the radius

$$
\mathfrak{R}_{\alpha_{n_{i}} k_{n_{i}}+1}{ }_{\beta_{n_{i}}} \rightarrow 0 .
$$

Since $\inf _{i}\left|\lambda_{n_{i}}\right|^{2}>c>1$, we have for large $i$ that the isometric circles of $\alpha_{n_{i}}^{k_{n_{i}}+1} \beta_{n_{i}}$ are disjoint and lies between $c^{-1}$ and $c$. In particular,

$$
c^{-1}<\left|z_{\alpha_{n_{i}}^{k n_{i}+1} \beta_{n_{i}, l}, l}\right| \leq\left|z_{\alpha_{n_{i}}^{k n_{i}+1} \beta_{n_{i}, u}}\right|<c,
$$

and $\left\langle\alpha_{n_{i}}, \alpha_{n_{i}}^{k_{n_{i}}+1} \beta_{n_{i}}\right\rangle$ satisfies Lemma 5.2.

Now consider Case $\left(\mathrm{B}_{2}^{\prime \prime}\right)$. First we define a new sequence of $\left\langle\tilde{\alpha}_{n}, \tilde{\beta}_{n}\right\rangle$ as follows: Consider $\left\langle\alpha_{n}, \alpha_{n}^{k_{n}} \beta_{n}\right\rangle$. If $\left|\operatorname{tr}\left(\alpha_{n}^{k_{n}} \beta_{n}\right)\right| \geq\left|\operatorname{tr}\left(\alpha_{n}\right)\right|$, then set $\tilde{\alpha}_{n}=\alpha_{n}, \tilde{\beta}_{n}=\beta_{n}$. Otherwise, 
let $\phi_{n}$ be the Mobius map so that $\phi_{n} \alpha_{n}^{k_{n}} \beta_{n} \phi_{n}^{-1}$ have fixed points $0, \infty$, and $\phi_{n} \alpha_{n} \phi_{n}^{-1}$ have $z_{\phi_{n} \alpha_{n} \phi_{n}^{-1}, u}=1$. Set $\alpha_{n, 1}=\phi_{n} \alpha_{n}^{k_{n}} \beta_{n} \phi_{n}^{-1}, \beta_{n, 1}=\phi_{n} \alpha_{n} \phi_{n}^{-1}$. We define integer $k_{n, 1}$ with respect to $\left\langle\alpha_{n, 1}, \beta_{n, 1}\right\rangle$ the same way as we defined $k_{n}$ before.

Now if $\left|\operatorname{tr}\left(\alpha_{n, 1}^{k_{n, 1}} \beta_{n, 1}\right)\right| \geq\left|\operatorname{tr}\left(\alpha_{n, 1}\right)\right|$ then we set $\tilde{\beta}_{n}=\beta_{n, 1}$ and $\tilde{\alpha}_{n}=\alpha_{n, 1}$. Otherwise, we repeat this construction to get a sequence $\left\langle\alpha_{n, m}, \beta_{n, m}\right\rangle$. By construction for a each $n$, either there exists a $m$ such that $\left|\operatorname{tr}\left(\alpha_{n, m}^{k_{n, m}} \beta_{n, m}\right)\right| \geq\left|\operatorname{tr}\left(\alpha_{n, m}\right)\right|$ or we have $\left|\operatorname{tr}\left(\alpha_{n, \eta_{n, m}}^{k_{n, m}} \beta_{n, m}\right)\right|<\left|\operatorname{tr}\left(\alpha_{n, m}\right)\right|$ for all $m$. Assume the latter holds, since $\alpha_{n, m+1}=$ $\phi_{n, m} \alpha_{n, m}^{k_{n, m}} \beta_{n, m} \phi_{n, m}^{-1}$ we have $\left|\operatorname{tr}\left(\alpha_{n, m+1}\right)\right|<\left|\operatorname{tr}\left(\alpha_{n, m}\right)\right|$ for all $m$. If $\lim _{m}\left|\operatorname{tr}\left(\alpha_{n, m}\right)\right|=$ 0 then take $m_{n}$ to be the first integer $m$ with $\left|\operatorname{tr}\left(\alpha_{n, m}\right)\right|<1 / n$. If $\lim _{m}\left|\operatorname{tr}\left(\alpha_{n, m}\right)\right|>0$ then take $m_{n}$ to be the first integer $m$ with $\left|\operatorname{tr}\left(\alpha_{n, m+1}\right)\right|>\left|\operatorname{tr}\left(\alpha_{n, m}\right)\right|-1 / n$. If the former holds, we set $m_{n}$ to be the first integer $m$ with $\left|\operatorname{tr}\left(\alpha_{n, m}^{k_{n, m}} \beta_{n, m}\right)\right| \geq\left|\operatorname{tr}\left(\alpha_{n, m}\right)\right|$. Hence there exists a $m_{n}$ such that either $\left|\operatorname{tr}\left(\alpha_{n, m_{n}}^{k_{n, m_{n}}} \beta_{n, m_{n}}\right)\right|>\left|\operatorname{tr}\left(\alpha_{n, m_{n}}\right)\right|-1 / n$, or $\left|\operatorname{tr}\left(\alpha_{n, m_{n}}\right)\right|<1 / n$. We define $\tilde{\alpha}_{n}=\alpha_{n, m_{n}}, \tilde{\beta}_{n}=\beta_{n, m_{n}}$.

Now consider $\left\langle\tilde{\alpha}_{n}, \tilde{\beta}_{n}\right\rangle$. If $\liminf _{n}\left|\operatorname{tr}\left(\tilde{\alpha}_{n}\right)\right|<\infty$, we choose a subsequence with $\left|\operatorname{tr}\left(\tilde{\alpha}_{n_{i}}\right)\right|<c$ for all large $i$ and some $c>0$. Let $p_{i}$ be a sequence of least positive integers such that $\left|\operatorname{tr}\left(\tilde{\alpha}_{n_{i}}^{p_{i}} \tilde{\beta}_{n_{i}}\right)\right|>1 / D_{n_{i}}$. We conjugate $\tilde{\alpha}_{n_{i}}^{p_{i}} \tilde{\beta}_{n_{i}}$ by $\psi_{i}$ that fixes $0, \infty$ and $z_{\psi_{i}} \tilde{\alpha}_{n_{i}}^{p_{i}} \tilde{\beta}_{n_{i}} \psi_{i}^{-1}, u=1$. Set $\bar{\alpha}_{i}=\psi_{i} \tilde{\alpha}_{n_{i}} \psi_{i}^{-1}, \bar{\beta}_{i}=\psi_{i} \tilde{\alpha}_{n_{i}}^{p_{i}} \tilde{\beta}_{n_{i}} \psi_{i}^{-1}$. By construction, if $z_{\bar{\alpha}_{i}, l} \psi_{i} \rightarrow 0$ then $\left\langle\bar{\alpha}_{i}, \bar{\beta}_{i}\right\rangle$ satisfies $\left(\mathrm{B}_{1}\right)$, and if $z_{\bar{\alpha}_{i}, l} \rightarrow 1$ then $\left\langle\bar{\alpha}_{i}, \bar{\beta}_{i}\right\rangle$ satisfies (A). Otherwise there exists $\epsilon>0$ such that $\epsilon<\left|z_{\bar{\alpha}_{i}, l}\right|<1-\epsilon$ which implies that $Z_{\left\langle\bar{\alpha}_{i}, \bar{\beta}_{i}\right\rangle}>c$ for some $c>0$. Hence in either case, we are done.

On the other hand, suppose $\liminf _{n}\left|\operatorname{tr}\left(\tilde{\alpha}_{n}\right)\right|=\infty$. Since $\left|\operatorname{tr}\left(\tilde{\beta}_{n}\right)\right| \geq\left|\operatorname{tr}\left(\tilde{\alpha}_{n}\right)\right|$ then it's sufficient to assume that $\left\langle\tilde{\alpha}_{n}, \tilde{\beta}_{n}\right\rangle$ satisfies Case $\left(\mathrm{B}_{2}^{\prime \prime}\right)$, otherwise we are done. We define $\tilde{k}_{n}=k_{n, m_{n}}$.

Set $v_{n}=\tilde{\alpha}_{n}^{\tilde{k}_{n}} \tilde{\beta}_{n}, \mu_{n}=\tilde{\alpha}_{n}$. Since $\left|\operatorname{tr}\left(\tilde{\beta}_{n}\right)\right| \geq\left|\operatorname{tr}\left(\tilde{\alpha}_{n}\right)\right|$ and $\left|z_{\tilde{\beta}_{n},-}-z_{\tilde{\beta}_{n},+}\right| \leq 1+\delta_{n}$ with $\delta_{n} \rightarrow 0$ (this follows from $z_{\tilde{\beta}_{n}, u}=1, z_{\tilde{\beta}_{n}, l} \rightarrow 0$ ), it follows from Lemma 4.2 with Remark 4.5 and $\left|\operatorname{tr}\left(\alpha_{n, m_{n}}^{k_{n, m_{n}}} \beta_{n, m_{n}}\right)\right|>\left|\tilde{\beta}_{\tilde{\lambda}}\left(\alpha_{n, m_{n}}\right)\right|-1 / n$ which implies $\lim _{n}\left|\operatorname{tr}\left(v_{n}\right)\right| /\left|\tilde{\lambda}_{n}\right|>$ $\epsilon$ for $\epsilon>0$, we have $\left|\eta_{v_{n}}-1\right|<\delta\left|\tilde{\lambda}_{n}\right|^{-2}$, for some $\delta>0$. Since $\eta_{\nu_{n} \mu_{n}}=\eta_{v_{n}} \tilde{\lambda}_{n}^{-2}$ and $\eta_{\mu_{n} v_{n}}=\eta_{v_{n}}$, we have $\left|\eta_{v_{n} \mu_{n}}-\tilde{\lambda}_{n}^{-2}\right|=\left|\eta_{v_{n}} \tilde{\lambda}_{n}^{-2}-\tilde{\lambda}_{n}^{-2}\right|<\delta\left|\tilde{\lambda}_{n_{n}}\right|^{-4}$ and $\left|\eta_{\mu_{n} v_{n}}-1\right|=$ $\left|\eta_{v_{n}}-1\right|<\delta\left|\tilde{\lambda}_{n_{n}}\right|^{-2}$, for large $n$

We have two cases to consider:

$$
\begin{aligned}
& \lim _{n} \frac{\left|\tilde{\zeta}_{n} \tilde{\lambda}_{n}^{2 \tilde{k}_{n}}\right|-\left|\tilde{\lambda}_{n}\right|^{-2}}{\left|\tilde{\lambda}_{n}\right|^{-2}}=\infty \\
& \lim _{i} \frac{\left|\tilde{\zeta}_{n_{i}} \tilde{\lambda}_{n_{i}}^{2 \tilde{k}_{n_{i}}}\right|-\left|\tilde{\lambda}_{n_{i}}^{-2}\right|}{\left|\tilde{\lambda}_{n_{i}}\right|^{-2}}<\sigma \quad \text { for some subsequence } n_{i} .
\end{aligned}
$$


Proof assuming (2) Since $\left|\tilde{\zeta}_{n_{i}} \tilde{\lambda}_{n_{i}}^{2 \tilde{k}_{n_{i}}}\right|>\left|\tilde{\lambda}_{n_{i}}\right|^{-2}$ and (2) holds, and $\zeta_{\mu_{i} \nu_{i}}=\tilde{\lambda}_{n_{i}}^{2} \zeta_{v_{i}}$, we have for large $i$,

$$
1<\left|\zeta_{\mu_{i} \nu_{i}}\right|<(\sigma+1)
$$

Since we also have

$$
1-\delta\left|\tilde{\lambda}_{n_{i}}^{-2}\right|<\left|\eta_{\mu_{i} \nu_{i}}\right|<1+\delta\left|\tilde{\lambda}_{n_{i}}^{-2}\right|
$$

and $\left|\tilde{\lambda}_{n_{i}}\right| \rightarrow \infty$ it follows that there exists $\kappa>1$ such that $1-\kappa^{-1}<\left|\eta_{\mu_{i} \nu_{i}}\right|,\left|\zeta_{\mu_{i} \nu_{i}}\right|<$ $1+\kappa$ for large $i$. By Lemma 4.2 with Remark 4.5 and $\left|\operatorname{tr}\left(v_{i}\right)\right|>\epsilon\left|\tilde{\lambda}_{n_{i}}\right|$ for large $i$ we have for some $\rho_{1}, \rho_{2}>0$ that

$$
1-\kappa^{-1}-\rho_{1}\left|\tilde{\lambda}_{n_{i}}\right|^{-1}<\left|z_{\mu_{i} \nu_{i}, l}\right|,\left|z_{\mu_{i} \nu_{i}, u}\right|<1+\kappa+\rho_{2}\left|\tilde{\lambda}_{n_{i}}\right|^{-1} .
$$

Hence there exists $\kappa^{\prime}>1$ such that $\kappa^{\prime-1}<\left|z_{\mu_{i} \nu_{i}, l}\right| \leq\left|z_{\mu_{i} \nu_{i}, u}\right|<\kappa^{\prime}$.

If $\left|\operatorname{tr}\left(\mu_{i} v_{i}\right)\right| \rightarrow \infty$, then $\left\langle\mu_{i}, \mu_{i} \nu_{i}\right\rangle$ satisfies the second set of conditions of Lemma 5.2 , hence is classical.

If $\lim \sup \left|\operatorname{tr}\left(\mu_{i} v_{i}\right)\right|<\infty$, then define Mobius transformations $\psi_{i}$ such that $\psi_{i} \mu_{i} \nu_{i} \psi^{-1}$ have fixed points $0, \infty$ and $z_{\psi_{i} \mu_{i} \psi_{i}^{-1}, u}=1$. If $z_{\psi_{i} \mu_{i} \psi_{i}^{-1, l}} \rightarrow 0$ or $z_{\psi_{i} \mu_{i} \psi_{i}^{-1, l}} \rightarrow 1$ then $\psi_{i}\left\langle\mu_{i}, \mu_{i} \nu_{i}\right\rangle \psi_{i}^{-1}$ satisfies (A) or $\left(\mathrm{B}_{1}\right)$. Otherwise we have for some $\epsilon>0$ such that $\epsilon<\left|z_{\psi_{i} \mu_{i} \psi^{-1}, l}\right|<1-\epsilon$, which implies that $Z_{\psi_{i}\left\langle\mu_{i}, \mu_{i} \nu_{i}\right\rangle \psi_{i}^{-1}}>c$ for some $c>0$. This completes our proof of $\left(\mathrm{B}_{2}^{\prime \prime}\right)$ with (2).

Proof assuming (1) By (1), there exists $0<\rho_{n} \rightarrow \infty$ with $\rho_{n}<\left|\tilde{\lambda}_{n}\right|$ such that $\left|\tilde{\zeta}_{n} \tilde{\lambda}_{n}^{2 \tilde{k}_{n}}\right|-\left|\tilde{\lambda}_{n}\right|^{-2}>\rho_{n}\left|\tilde{\lambda}_{n}\right|^{-2}$. Let $\chi_{n}$ be Mobius transformations defined by $\chi_{n}(x)=$ $\left(\tilde{\lambda}_{n} / \sqrt{\rho_{n}}\right) x$. We will show that $\chi_{n}\left\langle\mu_{n}, v_{n}\right\rangle \chi_{n}^{-1}$ satisfies Remark 5.3 of Lemma 5.2.

Since $\left|\eta_{v_{n}}-1\right|<\delta /\left|\tilde{\lambda}_{n}\right|^{2}$ we have

$$
\frac{\left|\tilde{\lambda}_{n}\right|}{\sqrt{\rho_{n}}}-\frac{\delta}{\left|\tilde{\lambda}_{n}\right| \sqrt{\rho_{n}}}<\left|\eta_{\chi_{n} v_{n} \chi_{n}^{-1}}\right|<\frac{\left|\tilde{\lambda}_{n}\right|}{\sqrt{\rho_{n}}}+\frac{\delta}{\left|\tilde{\lambda}_{n}\right| \sqrt{\rho_{n}}} .
$$

By the condition of $\left(\mathrm{B}_{2}\right)$ we have $\left|\tilde{\zeta}_{n} \tilde{\lambda}_{n}^{2 \tilde{k}_{n}}\right|<1$. This gives,

$$
\frac{\sqrt{\rho_{n}}}{\left|\tilde{\lambda}_{n}\right|}+\frac{1}{\left|\tilde{\lambda}_{n}\right| \sqrt{\rho_{n}}}<\left|\zeta_{\chi_{n} v_{n} \chi_{n}^{-1}}\right|<\frac{\left|\tilde{\lambda}_{n}\right|}{\sqrt{\rho_{n}}}
$$

By Lemma 4.2 with Remark 4.5, $\left|z_{v_{i}, \pm}-\zeta_{v_{n}}\right|<\sigma\left|\operatorname{tr}\left(v_{n}\right)\right|^{-2}$ and $\left|z_{v_{i}}, \mp-\eta_{v_{n}}\right|<$ $\sigma\left|\operatorname{tr}\left(v_{n}\right)\right|^{-2}$ for some $\sigma>0$. Since $\left|\operatorname{tr}\left(v_{n}\right)\right|>\epsilon\left|\tilde{\lambda}_{n}\right|$ for large $n$ we have

$$
\left|z_{\chi_{n} v_{n} \chi_{n}^{-1}, \pm}-\eta_{\chi_{n} v_{n} \chi_{n}^{-1}}\right|<\frac{\sigma}{\epsilon\left|\tilde{\lambda}_{n}\right| \sqrt{\rho_{n}}}, \quad\left|z_{\chi_{n} v_{n} \chi_{n}^{-1}, \mp}-\zeta_{\chi_{n} v_{n} \chi_{n}^{-1}}\right|<\frac{\sigma}{\epsilon\left|\tilde{\lambda}_{n}\right| \sqrt{\rho_{n}}} .
$$


Hence

$$
\begin{aligned}
\left|z_{\chi_{n} v_{n} \chi_{n}{ }^{-1}, u}\right| & <\frac{\left|\tilde{\lambda}_{n}\right|}{\sqrt{\rho_{n}}}+\frac{\delta}{\left|\tilde{\lambda}_{n}\right| \sqrt{\rho_{n}}}+\frac{\sigma}{\epsilon\left|\tilde{\lambda}_{n}\right| \sqrt{\rho_{n}}}, \\
\left|z_{\chi_{n} v_{n} \chi_{n}^{-1}, l}\right| & >\frac{\sqrt{\rho_{n}}}{\left|\tilde{\lambda}_{n}\right|}-\frac{\delta}{\left|\tilde{\lambda}_{n}\right| \sqrt{\rho_{n}}}-\frac{\sigma}{\epsilon\left|\tilde{\lambda}_{n}\right| \sqrt{\rho_{n}}} .
\end{aligned}
$$

We have $\left|\tilde{\lambda}_{n}\right|^{-1}<\left|z_{\chi_{n} v_{n} \chi_{n}^{-1}, l}\right| \leq\left|z_{\chi_{n} v_{n} \chi_{n}^{-1}, u}\right|<\left|\tilde{\lambda}_{n}\right|$ for large $n$.

By above estimates for fixed points of $\chi_{n} v_{n} \chi_{n}^{-1}$, and $\left|\operatorname{tr}\left(v_{n}\right)\right|>\epsilon\left|\tilde{\lambda}_{n}\right|$ we have

$$
\begin{aligned}
\frac{\left(\left|z_{\chi_{n} v_{n} \chi_{n}^{-1}, u}\right|+1\right)\left(\left|\tilde{\lambda}_{n}\right|+1\right)}{\left|\operatorname{tr}\left(v_{n}\right)\right|\left(\left|\tilde{\lambda}_{n}\right|-\left|z_{\chi_{n} v_{n} \chi_{n}{ }^{-1}, u}\right|\right)} & <\frac{\left|\tilde{\lambda}_{n}\right|^{2} / \sqrt{\rho_{n}}+\left|\tilde{\lambda}_{n}\right| / \sqrt{\rho_{n}}+\left|\tilde{\lambda}_{n}\right|+\delta^{\prime}}{\epsilon\left|\tilde{\lambda}_{n}\right|^{2}\left(1-1 / \rho_{n}-\delta /\left(\left|\tilde{\lambda}_{n}\right|^{2} \sqrt{\rho_{n}}\right)-\sigma /\left(\epsilon\left|\tilde{\lambda}_{n}\right|^{2} \sqrt{\rho_{n}}\right)\right)} \\
& <\frac{1+1 /\left|\tilde{\lambda}_{n}\right|+\sqrt{\rho_{n}} /\left|\tilde{\lambda}_{n}\right|+\delta^{\prime} \sqrt{\rho_{n}} /\left|\tilde{\lambda}_{n}\right|^{2}}{\epsilon \sqrt{\rho_{n}}\left(1-1 / \rho_{n}-\delta /\left(\left|\tilde{\lambda}_{n}\right|^{2} \sqrt{\rho_{n}}\right)-\sigma /\left(\epsilon\left|\tilde{\lambda}_{n}\right|^{2} \sqrt{\rho_{n}}\right)\right)}
\end{aligned}
$$

and by $\rho_{n}<\left|\tilde{\lambda}_{n}\right|$ we have some $\delta^{\prime \prime}>1$ such that

$$
\begin{aligned}
& <\frac{\delta^{\prime \prime}}{\epsilon \sqrt{\rho_{n}}\left(1-1 / \rho_{n}-\delta /\left(\left|\tilde{\lambda}_{n}\right|^{2} \sqrt{\rho_{n}}\right)-\sigma /\left(\epsilon\left|\tilde{\lambda}_{n}\right|^{2} \sqrt{\rho_{n}}\right)\right)} \\
& \rightarrow 0 .
\end{aligned}
$$

For the other part of the conditions of Remark 5.3 we have:

If $\left|z_{\chi_{n} v_{n} \chi_{n}^{-1}, l}\right|<M$ then,

$$
\begin{aligned}
& \frac{\left(\left|z_{\chi_{n} v_{n} \chi_{n}^{-1}, l}\right|+1\right)\left(\left|\tilde{\lambda}_{n}\right|^{-1}+1\right)}{\left|\operatorname{tr}\left(v_{n}\right)\right|\left(\left|z_{\chi_{n} v_{n} \chi_{n}^{-1}, l}\right|-\left|\tilde{\lambda}_{n}\right|^{-1}\right)} \\
& \quad<\frac{(M+1)\left(\left|\tilde{\lambda}_{n}\right|^{-1}+1\right)}{\epsilon\left|\tilde{\lambda}_{n}\right|\left(\sqrt{\rho_{n}} /\left|\tilde{\lambda}_{n}\right|-\delta /\left(\left|\tilde{\lambda}_{n}\right| \sqrt{\rho_{n}}\right)-\sigma /\left(\epsilon\left|\tilde{\lambda}_{n}\right| \sqrt{\rho_{n}}\right)-1 /\left|\tilde{\lambda}_{n}\right|\right)} \\
& \quad<\frac{M^{\prime}}{\epsilon\left(\sqrt{\rho_{n}}-\delta / \sqrt{\rho_{n}}-\sigma /\left(\epsilon \sqrt{\rho_{n}}\right)-1\right)}<\frac{M^{\prime}}{\epsilon^{\prime \prime} \sqrt{\rho_{n}}} \rightarrow 0 .
\end{aligned}
$$

Otherwise we have $\left|z_{\chi_{n} v_{n} \chi_{n}^{-1}, l}\right| \rightarrow \infty$ and

$$
\frac{\left(\left|z_{\chi_{n} v_{n} \chi_{n}^{-1}, l}\right|+1\right)\left(\left|\tilde{\lambda}_{n}\right|^{-1}+1\right)}{\left|\operatorname{tr}\left(v_{n}\right)\right|\left(\left|z_{\chi_{n} v_{n} \chi_{n}^{-1}, l}\right|-\left|\tilde{\lambda}_{n}\right|^{-1}\right)}<\frac{\delta^{\prime \prime}}{\epsilon\left|\tilde{\lambda}_{n}\right|} \rightarrow 0 \quad \text { for some } \delta^{\prime \prime}>0
$$


Hence $\chi_{n}\left\langle\mu_{n}, v_{n}\right\rangle \chi_{n}^{-1}$ satisfies

$$
\begin{gathered}
\left|\tilde{\lambda}_{n}\right|^{-1}<\left|z_{\chi_{n} v_{n} \chi_{n}^{-1}, l}\right| \leq\left|z_{\chi_{n} v_{n} \chi_{n}^{-1}, u}\right|<\left|\tilde{\lambda}_{n}\right|, \\
\lim _{n}\left\{\frac{\left(\left|z_{\chi_{n} v_{n} \chi_{n}^{-1}, u}\right|+1\right)\left(\left|\tilde{\lambda}_{n}\right|+1\right)}{\left|\operatorname{tr}\left(v_{n}\right)\right|\left(\left|\tilde{\lambda}_{n}\right|-\left|z_{\chi_{n} v_{n} \chi_{n}^{-1}, u}\right|\right)}, \frac{\left(\left|z_{\chi_{n} v_{n} \chi_{n}^{-1}, l}\right|+1\right)\left(\left|\tilde{\lambda}_{n}\right|^{-1}+1\right)}{\left|\operatorname{tr}\left(v_{n}\right)\right|\left(\left|z_{\chi_{n} v_{n} \chi_{n}^{-1}, l}\right|-\left|\tilde{\lambda}_{n}\right|^{-1}\right)}\right\}=0,
\end{gathered}
$$

the conditions of Remark 5.3.

Hence we have completed proof Theorem 7.1.

\section{Proof of Main Theorem}

Theorem 8.1 There exists $\epsilon>0$ such that every 2-generated Schottky group $\Gamma$ with $D_{\Gamma}<\epsilon$ is a classical Schottky group.

Proof This follows from Theorem 6.1 and Theorem 7.1.

Proof of Theorem 1.1 Let $\Gamma^{\prime}$ be a nonelementary finitely generated Kleinian group. Selberg's lemma implies $\Gamma^{\prime}$ contains a torsion-free subgroup $\Gamma^{\prime \prime}$ of finite index, in particular $\mathfrak{D}_{\Gamma^{\prime}}=\mathfrak{D}_{\Gamma^{\prime \prime}}$.

Note that if $\Gamma^{\prime \prime}$ is geometrically infinite with $\Omega_{\Gamma^{\prime \prime}} \neq \varnothing$ then $D_{\Gamma^{\prime \prime}}=2$, this implies $\mathfrak{D}_{\Gamma^{\prime \prime}}=2$ for geometrically infinite groups. So we can assume $\Gamma^{\prime \prime}$ is geometrically finite when $\mathfrak{D}_{\Gamma^{\prime \prime}}<2$.

If $\Gamma^{\prime \prime}$ contains parabolic of rank $l_{\Gamma^{\prime \prime}}$ then $D_{\Gamma^{\prime \prime}} \geq l_{\Gamma^{\prime \prime}} / 2$. Hence, for sufficiently small Hausdorff dimension $D_{\Gamma^{\prime \prime}}$, we can assume $\Gamma^{\prime \prime}$ is convex-cocompact of second kind.

It follows from Ahlfors' finiteness theorem, that $\Omega_{\Gamma^{\prime \prime}} / \Gamma^{\prime \prime}$ consists of finite number of compact Riemann surfaces. Let $S$ be a component of $\Omega_{\Gamma^{\prime \prime}} / \Gamma^{\prime \prime}$. If $S$ is incompressible then $\pi_{1}(S)$ is a surface subgroup of $\Gamma^{\prime \prime}$. Since $1=D_{\pi_{1}(S)} \leq D_{\Gamma^{\prime \prime}}$, if $D_{\Gamma^{\prime \prime}} i 1$, we may assume $S$ is compressible. So we can decompose $\Gamma^{\prime \prime}$ along the compression disk. After repeating the decomposition process finitely many times we are left with topological balls, ie $\mathbb{H}^{3} / \Gamma^{\prime \prime}$ is a handle body. This implies $\Gamma^{\prime \prime}$ is a finitely generated free purely loxodromic Kleinian group of second kind, ie $\Gamma^{\prime \prime}$ is a Schottky group.

By assuming the limit set have sufficiently small Hausdorff dimension we have reduced the general case to the case of Schottky groups. Now it follows from Marden's rigidity theorem, all Schottky groups of the same rank are quasiconformally equivalent. Therefore we have from Theorem 8.1, there exists $\lambda>0$ such that all nonelementary finitely generated Kleinian $\Gamma^{\prime}$ with $\mathfrak{D}_{\Gamma^{\prime}} \leq \lambda$ contains a classical Schottky group of finite index. It follows that we have a strict lower bound on the Hausdorff dimension of all nonclassical Schottky group. 


\section{References}

[1] I Agol, Tameness of hyperbolic 3-manifolds arXiv:math.GT/0405568

[2] A F Beardon, The geometry of discrete groups, Graduate Texts in Math. 91, Springer, New York (1983) MR698777

[3] P G Doyle, On the bass note of a Schottky group, Acta Math. 160 (1988) 249-284 MR945013

[4] Y Hou, Critical exponent and displacement of negatively curved free groups, J. Differential Geom. 57 (2001) 173-193 MR1871493

[5] A Marden, Schottky groups and circles, from: "Contributions to analysis (a collection of papers dedicated to Lipman Bers)", (L V Ahlfors, I Kra, B Maskit, L Nirenberg, editors), Academic Press, New York (1974) 273-278 MR0361058

[6] D Mumford, C Series, D Wright, Indra's pearls. The vision of Felix Klein, Cambridge Univ. Press, New York (2002) MR1913879

[7] R S Phillips, P Sarnak, The Laplacian for domains in hyperbolic space and limit sets of Kleinian groups, Acta Math. 155 (1985) 173-241 MR806414

Department of Mathematics, North Dakota State University

Fargo, ND 58108, USA

yonghou@math.uic.edu

Proposed: Benson Farb

Seconded: Walter Neumann, Joan Birman
Received: 17 March 2008

Revised: 6 May 2009 